\title{
AN INVITATION TO MODEL THEORY AND C*-ALGEBRAS
}

\author{
MARTINO LUPINI
}

\begin{abstract}
We present an introductory survey to first order logic for metric structures and its applications to $\mathrm{C}^{*}$-algebras.
\end{abstract}

\section{INTRODUCTION}

This survey is designed as an introduction to the study of $\mathrm{C}^{*}$-algebras from the perspective of model theory for metric structures. The intended readership consists of anyone interested in learning about this subject, and naturally includes both logicians and operator algebraists. Considering this, we will not assume in these notes any previous knowledge of model theory, nor any in-depth knowledge of functional analysis, beyond a standard graduate-level course. A familiarity with $\mathrm{C}^{*}$-algebra theory and the classification programme [29] might be useful as a source of motivation and examples. Several facts from $\mathrm{C}^{*}$-algebra theory will be used, and detailed references provided. Most of the references will be to the comprehensive monographs [13, 70].

Logic for metric structures is a generalization of classical (or discrete) logic, suitable for applications to metric objects such as $\mathrm{C}^{*}$-algebras. The monograph [11] presents a quick but complete introduction to this subject, and explains how the fundamental results from classical model theory can be recast in the metric setting. The model-theoretic study of $\mathrm{C}^{*}$-algebras has been initiated in [38-40] where, in particular, it shown how $\mathrm{C}^{*}$-algebras fit into the framework of model theory for metric structures. The motivations behind this study are manifold. With no pretense of exhaustiveness, we attempt to illustrate some of them here.

The most apparent contribution of first-order logic is to provide a syntactic counterpart to the semantic construction of ultraproducts and ultrapowers: the notion of formulas. Formulas allow one to express the fundamental properties of ultrapowers of $\mathrm{C}^{*}$-algebras (saturation) and diagonal embeddings into the ultrapower (elementarity). These general principles underpin most of the applications of the ultraproduct construction in $\mathrm{C}^{*}$-algebra theory, as they have appeared in various places in the literature under various names-Kirchberg's $\varepsilon$-test, reindexing arguments, etc. Isolating such general principles provides a valuable service of clarification and uniformization in the development of $\mathrm{C}^{*}$-algebra theory. In particular, this allows one to distinguish between, on one hand, what is just an instance of "general nonsense" and, on the other, what is a salient point where $\mathrm{C}^{*}$-algebras theory is crucially used.

This abstract model-theoretic point of view also makes it easier to recognize analogies between different contexts. Furthermore, it provides a language to formalize such analogies as precise mathematical statements, rather than just intuitive ideas. For instance, this paradigm can be applied to some aspects of the equivariant theory of $\mathrm{C}^{*}$-algebras, which studies $\mathrm{C}^{*}$-algebras endowed with a group action ( $\mathrm{C}^{*}$-dynamical systems). At least when the acting group is compact or discrete, $\mathrm{C}^{*}$-dynamical systems fit in the setting of first-order logic [47]. Adopting this perspective, one can naturally and effortlessly transfer ideas and arguments from the nonequivariant theory, as long as these are presented in terms of model-theoretic notions and principles. An instance of this phenomenon is the general theory of strongly self-absorbing $\mathrm{C}^{*}$-algebras, which admits a natural model-theoretic treatment; see Section 6. The equivariant analog of such a notion has been recently introduced and studied by Szabó in a series of papers [74-76], where the theory is developed in close parallel to the nonequivariant setting.

Beyond the motivations above, model theory provides the right tools for the study of ultrapowers and central sequence algebras per se. Questions on the number of nonisomorphic ultrapowers arise naturally in operator algebra theory, and can be traced back to McDuff's study of central sequences in the context of factors

2000 Mathematics Subject Classification. Primary 03C07, 46L05; Secondary 03C20, 46L35.

Key words and phrases. C*-algebra, strongly self-absorbing, model theory for metric structures, ultrapoduct, ultrapower, axiomatizable, model-theoretic forcing, building models by games, omitting types.

This survey originates from the lecture notes for the masterclass on "Applications of Model Theory to Operator Algebras" given by the author at the University of Houston from July 31st to August 4th, 2017, and supported by the NSF grant DMS-1700316. We are grateful to the organizers, Mehrdad Kalantar and Mark Tomforde, as well as the Department of Mathematics at the University of Houston, for their hospitality in such an occasion. The author has also been partially supported by the NSF Grant DMS-1600186. 
[62]. Many of such questions have been answered in [38-40] through the application of rather general modeltheoretic principles. We will explore some of these problems in Section 5, focusing on the situation under the Continuum Hypothesis. Ultrapowers and central sequence algebras are in fact just some instances of naturally occurring "massive $\mathrm{C}^{*}$-algebras". Other examples are Calkin algebras and, more generally, corona algebras. Deep questions about such algebras have been recently addressed in [23, 26, 32, 33, 35, 41, 43, 44, 46, 67, 83]. Methods from model theory, set theory, and forcing are key components of this line of research.

A further thread of applications of model theory comes from the use of techniques for constructing "generic objects", and the potential of using these techniques to construct interesting new examples of $\mathrm{C}^{*}$-algebras. This is particularly relevant considering that one of the most important open problems in $\mathrm{C}^{*}$-algebra theory (the UCT problem) depends on the existence of other methods of constructing nuclear $\mathrm{C}^{*}$-algebras other than the standard constructions of $\mathrm{C}^{*}$-algebra theory; see Section 7. Using the techniques of model-theoretic forcing and building models by games, many deep open problems in operator algebra theory have been reformulated in terms of model-theoretic notions, in the hope that these might be more amenable to a direct attack. Examples of these problems include the famous Connes Embedding Problem and some its $\mathrm{C}^{*}$-algebraic counterparts (the Kirchberg Embedding Problem, the MF problem); see [36, 52, 54, 55].

While the list above does not exhaust the applications of model theory to operator algebras, we hope it will sufficiently motivate the choice of topics of this survey. After a general introduction to the logic for metric structures (Section 2), we will explain how many classes of $\mathrm{C}^{*}$-algebras can be described through formulas (Section 3). Ultraproducts and their model-theoretic properties are considered in Section 4, and the question on the number of ultraproducts in Section 5. The important class of strongly self-absorbing $\mathrm{C}^{*}$-algebra and its model-theoretic treatment is the subject of Section 6. We conclude in Section 7 with a quick introduction to the classification programme of nuclear $\mathrm{C}^{*}$-algebras, a description of the model-theoretic content of nuclearity and other regularity properties, and an outlook on the applications of model-theoretic forcing to produce interesting examples of nuclear $\mathrm{C}^{*}$-algebras.

No result presented in this survey is original, although the presentation of some of the material is new. The results concerning the general theory of first-order logic for metric structures can be found in [11, 39]. Axiomatizability of the classes of $\mathrm{C}^{*}$-algebras presented here and many more is contained in [19, 36], as well as the model-theoretic description of nuclearity and other regularity properties. The theorem on the number of nonisomorphic ultrapowers of $\mathrm{C}^{*}$-algebras is one of the main results of $[38,39]$, together with the corresponding fact for $\mathrm{II}_{1}$ factors. The model-theoretic treatment of strongly self-absorbing $\mathrm{C}^{*}$-algebras is the subject of [37]. The model-theoretic proof of the characterization of $D$-absorption presented here is in some respects original, although heavily inspired by the proofs from the literature [82, Theorem 2.2], especially those in the equivariant setting from [74]. Finally, the technique of model-theoretic forcing in the metric setting has been first considered in [12] and then further developed in [36, 42, 52].

Acknowledgments. We are grateful to Piotr Koszmider, Andrew Ostergaard, and Richard Timoney for their comments on a preliminary version of the present survey.

\section{FiRST-ORDER LOGIC FOR METRIC STRUCTURES}

2.1. Languages. Model theory focuses on the study of classes of objects, rather than single objects on their own. The important notion of language or signature has the purpose of formalizing the assertion that a certain class is made of objects "of the same kind". It also allows one to make explicit which operations on the given objects are being considered. Formally, a language (or signature) usually denoted by $L$, is a collection of symbols. These symbols are of two kinds: function symbols and relation symbols. Each function symbol $f$ in the language $L$ has attached a natural number $n_{f}$, called its arity, and a function $\varpi^{f}:[0,+\infty)^{n_{f}} \rightarrow[0,+\infty)$ continuous at 0 and vanishing at 0 , called its continuity modulus. Similarly, each relation symbol $R$ in the language $L$ has attached a natural number $n_{R}$, called its arity, a function $\varpi^{R}:[0,+\infty)^{n_{R}} \rightarrow[0,+\infty)$ continuous at 0 and vanishing at 0 , called its continuity modulus, and a compact internal $J_{R}$ of $\mathbb{R}$, called its bound. The language $L$ includes a distinguished relation symbol, called the metric symbol. This is denoted by $d$, and it has arity 2 , and continuity modulus $\varpi^{d}\left(t_{0}, t_{1}\right)=t_{0}+t_{1}$. As customary we will call binary a symbol of arity 2 , and unary a symbol of arity 1 . The arity $n_{f}$ of a function symbol $f$ is allowed to be 0 , in which case one says that $f$ is a constant symbol.

Given a language $L$, one can then define the notion of $L$-structure. Briefly, an $L$-structure is a set endowed with functions and relations corresponding to the symbols in $L$. Precisely, an $L$-structure is a complete metric space $\left(M, d^{M}\right)$ together with assignments $f \mapsto f^{M}$ and $R \mapsto R^{M}$, which assign to each function symbol $f$ in $L$ its interpretation $f^{M}$ in $M$, and to each relation symbol $R$ in $L$ its interpretation $R^{M}$ in $M$. These interpretations 
are required to satisfy the following properties. If $f$ is a function symbol of arity $n_{f}$ and continuity modulus $\varpi^{f}$, then $f^{M}$ is a function $f^{M}: M^{n_{f}} \rightarrow M$ such that, for every $\bar{a}, \bar{b} \in M^{n_{f}}, d^{M}(f(\bar{a}), f(\bar{b})) \leq \varpi^{f}\left(d^{M}(\bar{a}, \bar{b})\right)$. When the arity $n_{f}$ of $f$ is zero, i.e. when $f$ is a constant symbol, by convention the set $M^{n_{f}}$ consists of a single point, and the function $f^{M}: M^{n_{f}} \rightarrow M$ can be simply seen as a distinguished element of $M$. If $R$ is a relation symbol of arity $n_{R}$, continuity modulus $\varpi^{R}$, and bound $J^{R}$, then $R^{M}$ is a function $R^{M}: M^{n_{R}} \rightarrow J_{R} \subset \mathbb{R}$ such that, for every $\bar{a}, \bar{b} \in M^{n_{f}},|R(\bar{a})-R(\bar{b})| \leq \varpi^{R}\left(d^{M}(\bar{a}, \bar{b})\right)$. Furthermore, the interpretation of the metric symbol $d$ of $L$ is required to be equal to the metric $d^{M}$ of $M$ (consistently with the notation above).

To summarize, an $L$-structure is a space endowed with some extra operations (functions and relations) as prescribed by the language. Furthermore, the language contains names (or symbols) for such operations. This allows one to uniformly and unambiguously refer to such operations when considering the class of all $L$-structures.

2.2. Metric groups. At this point, examples are in order. We consider for now examples from metric geometry and group theory. The first natural example is the language $L$ containing no function symbol, and whose unique relation symbol is the distinguished symbol for the metric $d$. One also has to specify in $L$ a bound $J_{d}$ for $d$, which we can choose to be $[0,1]$. The continuity modulus for $d$ can be defined to be $\varpi^{d}\left(t_{0}, t_{1}\right)=t_{0}+t_{1}$. This completely defines a language $L$ in the sense above. It is clear that, for such a language, an $L$-structure is just a complete metric space $\left(M, d^{M}\right)$ whose metric attains values in [0,1]. Thus the class of $L$-structure consists of the class of complete metric spaces of diameter at most 1.

A slightly more sophisticated example can be obtained by adding to this language some function symbols, to describe some additional algebraic structure which might be present on a complete metric space. The first natural example is the case of a language $L$ containing a single binary function symbol, to describe a binary operation. Since we want to think of it as a binary operation, we denote such a binary function symbol by . , for which we use the usual infix notation. We also need to prescribe a continuity modulus for such a binary function symbol, which we define to be $\left(t_{0}, t_{1}\right) \mapsto t_{0}+t_{1}$. As above, we assume that the unique relation symbol in $L$ is the metric symbol. In this case, an $L$-structure is a complete metric space $\left(M, d^{M}\right)$ with diameter 1 endowed with a binary operation ${ }^{M}$. The choice of continuity modulus for the symbol $\cdot$ in $L$ forces ${ }^{M}$ to satisfy

$$
d^{M}\left(a_{0} \cdot{ }^{M} b_{0}, a_{1} \cdot{ }^{M} b_{1}\right) \leq d^{M}\left(a_{0}, a_{1}\right)+d^{M}\left(b_{0}, b_{1}\right) .
$$

Conversely, any complete metric space with diameter 1 endowed with a binary operation satisfying Equation (1) can be seen as an $L$-structure. In this case, the class of $L$-structures contains the important example of bi-invariant metric groups. A bi-invariant metric group is a complete metric space $\left(G, d^{G}\right)$ endowed with a group operation ${ }^{G}$ with the property that left and right translations, i.e. the maps $x \mapsto a x$ and $x \mapsto x a$ for $a \in G$, are isometries. It is clear from the discussion above that a bi-invariant metric group can be seen as a structure in the language $L$ just described.

Bi-invariant metric groups arise naturally in operator algebras, group theory, and metric geometry. For instance, for every $n \in \mathbb{N}$, the group $U_{n}$ of $n \times n$ unitary matrices is a bi-invariant metric group when endowed with the metric $d(u, v)=2^{-1 / 2}\|u-v\|_{2}$. Here $\|a\|_{2}$ denotes the normalized Hilbert-Schmidt norm (or Frobenius norm) $\tau\left(a^{*} a\right)^{1 / 2}$ of a matrix $a$, where $\tau$ is the canonical trace of $n \times n$ matrices, suitable normalized so that $\tau(1)=1$. This is a particular instance of a more general class of examples, arising from von Neumann algebra theory. If $M$ is a von Neumann algebra endowed with a faithful normalized trace $\tau$, then the unitary group $U(M)$ of $M$ is a bi-invariant metric group. The metric now is defined, as above, by $d(u, v)=\|u-v\|_{2}$, where $\|a\|_{2}$ denotes the 2-norm $\tau\left(a^{*} a\right)^{1 / 2}$ of $a$ with respect to the trace $\tau$. The case of unitary groups of matrices is recovered in the case when $M$ is a full matrix algebra.

One can also consider different metrics on the unitary group $U_{n}$. For instance, one can consider the metric $d(u, v)=\|u-v\|$ induced by the operator norm of matrices, which can be concretely defined as the largest singular value. It is clear that left and right translations in $U_{n}$ are isometries also with respect to this metric, and so it yields another example of bi-invariant metric group. Again, this is a particular instance of a more general class of examples arising from $\mathrm{C}^{*}$-algebra theory. Indeed, if $A$ is a $\mathrm{C}^{*}$-algebra, then one can consider the unitary group $U(A)$ as a bi-invariant metric group endowed with a metric induced by the norm of $A$.

Generalizing the examples above, one can consider an arbitrary unitary-invariant complete metric $d$ bounded by 1 on $M_{n}(\mathbb{C})$, and then endow the unitary group $U_{n}$ with the bi-invariant metric induced by $d$. Any choice of such a unitary-invariant metric gives rise to a different $L$-structure. Beside the ones considered above, an important example of unitary-invariant metric $d$ is the (normalized) rank metric, defined by $d(a, b)=$ $\operatorname{rank}(a-b) / n$, where rank denotes usual rank of matrices. Clearly, such a metric is not only unitary-invariant, 
but also invariant with respect to multiplication by arbitrary invertible matrices. Therefore, it defines an invariant metric also in the group $\mathrm{GL}_{n}(\mathbb{C})$ of invertible $n \times n$ complex matrices.

Another class of examples arises by considering, for $n \in \mathbb{N}$, the symmetric group $S_{n}$, consisting of permutations of the set $\{1,2, \ldots, n\}$. In this case, the bi-invariant metric is given by the (normalized) Hamming metric

$$
d(\sigma, \tau)=\frac{1}{n}|\{i \in\{1,2, \ldots, n\}: \sigma(i) \neq \tau(i)\}| .
$$

Finally, any (discrete) group $\Gamma$ can be regarded as a bi-invariant metric group with respect to the trivial $\{0,1\}$ valued metric defined by $d(g, h)=1$ whenever $g, h \in \Gamma$ are distinct.

We mention in passing that the classes of metric groups introduced here play a crucial role in defining and studying important regularity properties for countable discrete groups. Indeed, given a countable discrete group $\Gamma$ and a class $\mathcal{C}$ of bi-invariant metric groups, one says that $\Gamma$ is $\mathcal{C}$-approximable if there exist strictly positive real numbers $\delta_{g}$ for $g \in \Gamma \backslash\{1\}$ such that, for every finite subset $F$ of $\Gamma \backslash\{1\}$ and for every $\varepsilon>0$, there exists a bi-invariant metric group $\left(G, d^{G},{ }^{G}\right)$ in $\mathcal{C}$ and a function $\Phi: \Gamma \rightarrow G$ such that $\Phi(1)=1$, $d(\Phi(g h), \Phi(g) \Phi(h))<\varepsilon$, and $d(\Phi(g), 1)>\delta_{g}$ for every $g, h \in F[78$, Definition 1.6]. By varying the class $\mathcal{C}$, one obtains various regularity properties for countable discrete groups:

- when $\mathcal{C}$ is the class of permutation groups $S_{n}$ for $n \in \mathbb{N}$ endowed with the Hamming distance, a group is $\mathcal{C}$-approximable if and only if it is sofic $[18,56,65]$;

- when $\mathcal{C}$ is the class of unitary groups $U_{n}$ for $n \in \mathbb{N}$ endowed with the Hilbert-Schmidt distance, a group is $\mathcal{C}$-approximable if and only if it is hyperlinear, which is in turn equivalent to the assertion that the corresponding group von Neumann algebra satisfies the Connes Embedding Problem [63];

- when $\mathcal{C}$ is the class of unitary groups $U_{n}$ for $n \in \mathbb{N}$ endowed with the operator norm, a group is $\mathcal{C}$ approximable if and only if it is matricially finite or $M F[14,20]$, which is in turn equivalent to the assertion that the corresponding group $\mathrm{C}^{*}$-algebra is quasidiagonal [20, Theorem 2.8];

- when $\mathcal{C}$ is the class of groups $\mathrm{GL}_{n}(\mathbb{C})$ endowed with the rank metric, a group is $\mathcal{C}$-approximable if and only if it is linear sofic [3].

The interest on these properties is due on one hand to the fact that several long-standing open problems in group theory - such as Gottschalk's conjecture [56], Kaplansky's direct finiteness conjecture [27], and the algebraic eigenvalue conjecture [77] - have been settled for groups satisfying these extra regularity properties. On other hand, these extra assumptions seem to be very generous, to the point that no countable discrete group that does not satisfy any of the approximation properties mentioned above is currently known.

To conclude this detour on metric groups, and this list of examples of languages, we mention a language closely related to the one just considered. We let $L$ be a language consisting of a binary function symbol · as above, together with a unary function symbol suggestively denoted by inv with the identity map as continuity modulus, and a constant symbol 1. A bi-invariant metric group $G$ can be naturally regarded as a structure in this richer language $L$, where the interpretation of the unary function inv is just the function $g \mapsto g^{-1}$ assigning to an element of $G$ its inverse, and the constant symbol 1 is interpreted as the identity element of $G$. This example showcases an important point: a given object, such as a bi-invariant metric group, can be seen as a structure in possibly different languages. The choice of the language allows one to keep track of which operations one is considering.

2.3. Languages with domains of quantification. Ultimately, we would like to regard unital $\mathrm{C}^{*}$-algebras as structures in a suitable language $L$ containing symbols for all the $\mathrm{C}^{*}$-algebra operations. While not impossible, it is somewhat inconvenient to regard $\mathrm{C}^{*}$-algebras as structures in the restricted setting considered in Subsection 2.1. Naturally, such a language $L$ would contain binary function symbols + and · for sum and multiplication, a unary function symbol for the adjoint operation, constant symbols for the additive and multiplicative neutral elements, and a unary function symbols for the scalar multiplication function $x \mapsto \lambda x$ for any given $\lambda \in \mathbb{C}$, in addition to the distinguished metric symbol. However, in view of the requirements on bounds on relation symbols (including the metric symbol) one can only consider bounded metric spaces as structures. It is therefore natural to then restrict to the unit ball $A^{1}$ of a given $\mathrm{C}^{*}$-algebra $A$. This is not a real restriction, since it is clear that all the information about $A$ is already present in $A^{1}$. This however makes it problematic to have a function symbol for the addition operation, since $A^{1}$ is not invariant under addition. A solution to this would be replace the binary function symbol for addition with a binary function symbol to denote the average operation $(x, y) \mapsto(x+y) / 2$. (More generally, one could consider for $n \in \mathbb{N}$ and $\left(\lambda_{1}, \ldots, \lambda_{n}\right) \in \mathbb{C}^{n}$ such that $\sum_{i=1}^{n}\left|\lambda_{i}\right| \leq 1$, an $n$-ary function symbol for the function $\left(x_{1}, \ldots, x_{n}\right) \mapsto \lambda_{1} x_{1}+\cdots+\lambda_{n} x_{n}$.) While this is 
possible, the corresponding notion of structure that one obtains seems to be far from the way in which, in practice, $\mathrm{C}^{*}$-algebras are regarded as structures by $\mathrm{C}^{*}$-algebraists.

We pursue therefore a different path, which consists in introducing a more general framework than the one considered in Subsection 2.1. This is the framework of languages with domains of quantification. Briefly, in this setting one adds to the language a collection of symbols (domains of quantification) to be interpreted as closed subsets of the structure. In this case, all the requirements concerning the interpretations of function and relation symbols, including the boundedness requirement on the metric, are only imposed relatively to a given domain, or tuple of domains. This allows one consider structures, such as $\mathrm{C}^{*}$-algebras, where the metric is globally unbounded, although it is bounded when restricted to any given choice of domains of quantifications. In the case of $\mathrm{C}^{*}$-algebras, the domains of quantifications will be interpreted as the balls centered at the origin.

We now present the details. As we have just mentioned, in this setting, a language $L$ is endowed with a collection $\mathcal{D}$ of domains of quantification. The set $\mathcal{D}$ is endowed with an ordering, which is upward directed. In this case, for each function symbol $f$ in $L$ and for each choice of input domains $D_{1}, \ldots, D_{n_{f}}$, the language $L$ prescribes:

- its arity $n_{f}$;

- an output domain $D_{D_{1}, \ldots, D_{n_{f}}}^{f}$;

- a continuity modulus $\varpi_{D_{1}, \ldots, D_{n_{f}}}^{f}:[0,+\infty)^{n_{f}} \rightarrow[0,+\infty)$.

Similarly, for each relation symbol $R$ in $L$ and for each choice of input domains $D_{1}, \ldots, D_{n_{f}}$, the language $L$ prescribes:

- its arity $n_{R}$;

- a bound $J_{D_{1}, \ldots, D_{n_{f}}}^{R}$;

- a continuity modulus $\varpi_{D_{1}, \ldots, D_{n_{R}}}^{R}:[0,+\infty)^{n_{f}} \rightarrow[0,+\infty)$.

Again, the language $L$ is assumed to contain a distinguished binary function symbol $d$ (metric symbol).

In this case, an $L$-structure is a complete metric space $\left(M, d^{M}\right)$ together with assignments:

- $D \mapsto D^{M}$ from the set of domains of quantification in $L$ to the collection of closed subsets of $M$;

- $f \mapsto f^{M}$ from the set of function symbols in $L$ to the collection of functions $M^{n} \rightarrow M$ for $n \in \mathbb{N}$;

- $R \mapsto R^{M}$ from the set of relation symbols in $L$ to the collection of functions $M^{n} \rightarrow \mathbb{R}$ for $n \in \mathbb{N}$;

satisfying the following properties:

(1) the collection $\left\{D^{M}: D \in \mathcal{D}\right\}$ of closed subsets of $M$ has dense union;

(2) the assignment $D \mapsto D^{M}$ is order-preserving, where the collection of closed subsets of $M$ is ordered by inclusion;

(3) for every function symbol $f$ and choice of input domains $D_{1}, \ldots, D_{n_{f}}$, and $\bar{a}, \bar{b} \in D_{1} \times \cdots \times D_{n_{f}}$, one has that $f^{M}(\bar{a}) \in D_{D_{1}, \ldots, D_{n_{f}}}^{f}$ and $d^{M}(f(\bar{a}), f(\bar{b})) \leq \varpi_{D_{1}, \ldots, D_{n_{f}}}^{f}(d(\bar{a}, \bar{b}))$;

(4) for every relation symbol $R$ and choice of input domains $D_{1}, \ldots, D_{n_{R}}$, and $\bar{a}, \bar{b} \in D_{1} \times \cdots \times D_{n_{R}}$, one has that $R^{M}(\bar{a}) \in J_{D_{1}, \ldots, D_{n_{f}}}^{R}$ and $|R(\bar{a})-R(\bar{b})| \leq \varpi_{D_{1}, \ldots, D_{n_{f}}}^{f}(d(\bar{a}, \bar{b}))$.

We conclude by noting that any language as defined in Subsection 2.1 can be seen as a particular instance of a language with domains of quantification as defined in this section, by declaring that the set $\mathcal{D}$ of domains of quantification is a singleton $\{D\}$. In this case, we omit the reference to such a unique domain in the quantifiers, and write simply $\sup _{x}$ and $\inf _{x}$ instead of $\sup _{x \in D}$ and $\inf _{x \in D}$.

2.4. $\mathrm{C}^{*}$-algebras as structures. We are now ready to discuss how $\mathrm{C}^{*}$-algebras can be seen as structures in continuous logic, when one consider the framework introduced in Subsection 2.3. We briefly recall that a $\mathrm{C}^{*}$ algebra is, abstractly, a complex algebra $A$ endowed with an involution $a \mapsto a^{*}$ and a complete norm $a \mapsto\|a\|$ satisfying $\|a b\| \leq\|a\|\|b\|$ for $a, b \in A$, and the $\mathrm{C}^{*}$-identity $\left\|a^{*} a\right\|=\|a\|^{2}$ for $a \in A$. For every $n \in \mathbb{N}$, the algebraic tensor product $M_{n}(\mathbb{C}) \otimes A=M_{n}(A)$ is endowed with a canonical norm which turns into a $\mathrm{C}^{*}$ algebra. We will always regard $M_{n}(A)$ as a $\mathrm{C}^{*}$-algebra endowed with such a norm. We will only consider unital $\mathrm{C}^{*}$-algebras, which are moreover endowed with a multiplicative identity (unit) 1.

The Gelfand-Neimark theorem guarantees that one can concretely represent any (abstract) $\mathrm{C}^{*}$-algebras as a closed subalgebra of the algebra $B(H)$ of bounded linear operators on a Hilbert space $H$. In this case the involution is the map assigning to an operator its Hermitian adjoint, the norm is the operator norm given by $\|T\|=\sup \{\|T \xi\|: \xi \in H,\|\xi\| \leq 1\}$ for $T \in B(H)$, and 1 is the identity operator.

The language of $\mathrm{C}^{*}$-algebras $L^{\mathrm{C}^{*}}$ consists of: 
- a sequence $\left\{D_{n}: n \in \mathbb{N}\right\}$ of domains of quantifications, linearly ordered by setting $D_{n}<D_{m}$ if and only if $n<m$;

- binary function symbols for addition and multiplication;

- for every $\lambda \in \mathbb{C}$, a unitary function symbol for the scalar multiplication function $x \mapsto \lambda x$;

- constant symbols for 0 and 1 ;

- a unary relation symbol for the norm, as well as the metric symbol;

- for every $n \in \mathbb{N}$, a $n^{2}$-ary relation symbol for the norm of elements of $M_{n}(A)$.

We want to regard a $\mathrm{C}^{*}$-algebra $A$ as an $L^{\mathrm{C}^{*}}$-structure, where the domain $D_{n}$ is interpreted as the ball of $A$ of center 0 and radius $n$, the function and relation symbols are interpreted in the obvious way. Keeping this in mind, it is clear that one can define continuity moduli, output domains, and bounds for the given function and relation symbols in such a way that any $\mathrm{C}^{*}$-algebra meets the requirements that an $L^{\mathrm{C}^{*}}$-structure has by definition to satisfy. For example, let us consider the function symbol for multiplication, and the input domains $D_{n}$ and $D_{m}$. In this case, one can declare the output domain to be $D_{n m}$, and the continuity modulus to be the function $\left(t_{1}, t_{2}\right) \mapsto m t_{1}+n t_{2}$.

2.5. Formulas. One of the upshots of regarding a class of objects as structures in continuous logic is to obtain a corresponding notion of first-order property. These are the properties that can be expressed through formulas. We begin with the syntax of formulas, and describe how formulas in a given language are defined. So let us fix a language $L$, and define the notion of $L$-formula. Intuitively, an $L$-formula is an expression that describes a property of an $L$-structure, or of a tuple of elements of an $L$-structure, by only referring to the given $L$-structure, its elements, and its operations which are given by the interpretations of the function and relation symbols in $L$.

Before we introduce the notion of $L$-formula, we need to consider the notion of $L$-term. Informally, an $L$ term is an expression that described how elements of a given $L$-structure can be combined together by using the function symbols in $L$. To make this precise, we suppose that we have a collection of symbols, usually denoted by $x, y, z, \ldots$, possibly with decorations such as $x_{1}, x_{2}, x_{3}, \ldots$, which we call variables. Variables are used in the definition of terms and formulas, and they should be thought of as "place-holders", which can be possibly replaced by elements of a structure. (This is analogous to the role of variables $\bar{x}$ in a polynomial $p(\bar{x})$ with coefficients in a ring $R$. These variables "substituted" by elements of $R$ when considering the corresponding polynomial function. In fact, this usage of variables is a particular instance of the usage from model theory.) We assume that to each variable $x$ is uniquely attached a domain of quantification $D$, which we call the domain of $x$.

Now one can say briefly that an $L$-term is any expression that can be formed starting from variables and constant symbols, and applying function symbols from $L$. More extensively, one declare that:

- variables are $L$-terms;

- constant symbols are $L$-terms;

- if $t_{1}, \ldots, t_{n}$ are $L$-terms, and $f$ is an $n$-ary function symbol in $L$, then $\left(f\left(t_{1}, \ldots, t_{n}\right)\right)$ is an $L$-term.

Given an $L$-term $t$, one can speak of the variables that appear in $t$. Formally, if $x$ is a variable, one can define the property that $x$ appears in $t$ by induction on the complexity of $t$ as follows: if $t$ is a variable, then $x$ appears in $t$ if and only if $x$ is equal to $t$; if $t$ is a constant symbol then $x$ does not appear in $t$; if $t=f\left(t_{1}, \ldots, t_{n}\right)$ then $x$ appears in $t$ if and only if $x$ appears in $t_{i}$ for some $i \in\{1,2, \ldots, n\}$. One then write $t\left(x_{1}, \ldots, x_{k}\right)$ to denote the fact that the variables that appear in $t$ are within $x_{1}, \ldots, x_{k}$.

The notion of $L$-term allows one to easily define the notion of atomic $L$-formula. This is an expression $\varphi$ of the form $R\left(t_{1}, \ldots, t_{n}\right)$, where $R$ is an $n$-ary function symbol in $L$ and $t_{1}, \ldots, t_{n}$ are $L$-terms. If $t_{1}, \ldots, t_{n}$ are variables within $x_{1}, \ldots, x_{k}$, then one says that $\varphi$ has free variables within $x_{1}, \ldots, x_{k}$, and write $\varphi\left(x_{1}, \ldots, x_{k}\right)$.

Starting from the notion of atomic formula, one can define the arbitrary formulas. Informally, formulas are expressions obtained by combining atomic formulas by using logical connectives and quantifiers. In classical (discrete) first-order logic, the logical connectives are the usual symbols $\wedge, \vee, \neg, \rightarrow$, $\leftrightarrow$, which can be thought of as expressions to denote Boolean functions. In logic for metric structures, these Boolean functions are replaced with arbitrary continuous functions $q: \mathbb{R}^{d} \rightarrow \mathbb{R}$. On the side of quantifiers, in the discrete setting these are the expressions $\forall x$ and $\exists x$, where $x$ is a variable. In this case, one also says that $\forall x$ and $\exists x$ are quantifiers over $x$. In the continuous setting, for each domain of quantification and for each variable $x$ one has quantifiers $\sup _{x \in D}$ and $\inf _{x \in D}$ (this justifies the name of domains of quantification). One then formally defines $L$-formulas by induction as follows:

- atomic $L$-formulas are $L$-formulas;

- if $\varphi_{1}, \ldots, \varphi_{n}$ are $L$-formulas and $q: \mathbb{R}^{n} \rightarrow \mathbb{R}$ is a continuous function, then $q\left(\varphi_{1}, \ldots, \varphi_{n}\right)$ is an $L$-formula; 
- if $\varphi$ is an $L$-formula, and $x$ is a variable with domain $D$, then $\inf _{x \in D} \varphi$ and $\sup _{x \in D} \varphi$ are $L$-formulas.

For brevity, a sequence of quantifiers $\sup _{x_{1} \in D_{1}} \cdots \sup _{x_{n} \in D_{n}}$ is abbreviated by $\sup _{\bar{x} \in \bar{D}}$ where $\bar{x}$ is the tuple of variables $\left(x_{1}, \ldots, x_{n}\right)$ and $\bar{D}$ is the corresponding tuple of domains $\left(D_{1}, \ldots, D_{n}\right)$.

The variables that appear in an $L$-formula can be bound or free, depending on whether they are in the scope of a quantifier over them or not. Formally, one declares when a variable $x$ appears freely in $\varphi$ by induction on the complexity of $\varphi$ as follows:

- if $\varphi$ is an atomic formula $R\left(t_{1}, \ldots, t_{n}\right)$, then $x$ appears freely in $\varphi$ iff it appears in $t_{i}$ for some $i \in$ $\{1,2, \ldots, n\}$;

- $x$ appears freely in $q\left(\varphi_{1}, \ldots, \varphi_{n}\right)$ iff it appears freely in $\varphi_{i}$ for some $i \in\{1,2, \ldots, n\}$;

- $x$ appears freely in $\inf _{y \in D} \varphi$ or $\sup _{y \in D} \varphi$ iff $x$ appears freely in $\varphi$ and $x$ is different from $y$.

If the free variables of $\varphi$ are within $x_{1}, \ldots, x_{k}$, then we write $\varphi\left(x_{1}, \ldots, x_{k}\right)$. In this case, $\varphi$ should be thought of as an expression describing how close a given $k$-tuple of elements of an $L$-structure is to satisfy a certain property. When $\varphi$ has no free variables, one says that $\varphi$ is an $L$-sentence. In this case, $\varphi$ should be thought of as an expression describing how close a given structure is to satisfying a certain property.

As mentioned above, the purpose of formulas is to describe properties of structures, or elements of structures. This is made precise by the semantic notion of interpretation of a formula in a given structure. We begin with the interpretation of an $L$-term $t\left(x_{1}, \ldots, x_{k}\right)$ in an $L$-structure $M$, which is going to be a function $t^{M}$ : $D_{1}^{M} \times \cdots \times D_{k}^{M} \rightarrow M, \bar{a} \mapsto t^{M}(\bar{a})$, where $\left(D_{1}, \ldots, D_{k}\right)$ are the domains of $\left(x_{1}, \ldots, x_{k}\right)$. Briefly, this is defined by replacing the variables of $t$ with the given tuple $\bar{a}$ of elements of $M$, and by replacing constant symbols and function symbols $f$ with their interpretations. Formally, this is defined, again, by induction of the complexity, as follows:

- if $t(\bar{x})=x_{i}$ for some $i \in\{1,2, \ldots, k\}$, then $t^{M}(\bar{a})=a_{i}$;

- if $t$ is a constant symbol $c$, then $t^{M}(\bar{a})=c^{M}$;

- if $t=f\left(t_{1}, \ldots, t_{n}\right)$, then $t^{M}(\bar{a})=f^{M}\left(t_{1}^{M}(\bar{a}), \ldots, t_{n}^{M}(\bar{a})\right)$.

Starting from the interpretations of terms, one can define the interpretation of an $L$-formula $\varphi\left(x_{1}, \ldots, x_{k}\right)$, which is going to be a function $\varphi^{M}: D_{1}^{M} \times \cdots \times D_{k}^{M} \rightarrow \mathbb{R}, \bar{a} \mapsto \varphi^{M}(\bar{a})$. As above, this can be briefly defined by replacing all the terms, relation symbols, and domains of quantifications that appear, with their interpretation in $M$. Precisely, one can define this by induction on the complexity, as follows:

- if $\varphi$ is the atomic formula $R\left(t_{1}, \ldots, t_{n}\right)$, then $\varphi^{M}(\bar{a})=R^{M}\left(t_{1}(\bar{a}), \ldots, t_{n}(\bar{a})\right)$;

- if $\varphi$ is equal to $q\left(\varphi_{1}, \ldots, \varphi_{n}\right)$, then $\varphi^{M}(\bar{a})=q\left(\varphi_{1}^{M}(\bar{a}), \ldots, \varphi_{n}^{M}(\bar{a})\right)$;

- if $\varphi$ is equal to $\inf _{x \in D} \psi$, then $\varphi^{M}(\bar{a})=\inf _{x \in D^{M}} \psi^{M}(\bar{a})$, and similarly with sup.

Clearly, when $\varphi$ is an $L$-sentence, its interpretation $\varphi^{M}$ can be seen simply as a single real number. At this point, let us pause and see what the notions of terms and formulas just introduced correspond in the examples of languages that we have seen so far.

When $L$ is the language only containing the metric symbol, the only terms are just single variables, and the only atomic formulas are of the form $d(x, y)$ where $x, y$ are variables. The interpretation of such a formula in a complete metric space $\left(M, d^{M}\right)$ is the function $M \times M \rightarrow \mathbb{R},(a, b) \mapsto d^{M}(a, b)$. In this case, an example of sentence is the formula $\varphi$ given by $\sup _{x} \sup _{y} d(x, y)$. The interpretation of $\varphi$ is a metric $\operatorname{space}\left(M, d^{M}\right)$ is, clearly, the diameter of $M$.

A slightly more interesting example comes from considering the language $L$ for bi-invariant metric groups. In this case, a term in the free variables $x_{1}, \ldots, x_{n}$ can be seen as a parenthesized word in the variables $x_{1}, \ldots, x_{n}$, such as $\left(x_{1} \cdot\left(x_{2} \cdot x_{3}\right)\right)$. (Formally, the terms $\left(x_{1} \cdot\left(x_{2} \cdot x_{3}\right)\right)$ and $\left(\left(x_{1} \cdot x_{2}\right) \cdot x_{3}\right)$ are distinct terms, although they have the same interpretation in any bi-invariant metric group, or more generally in any $L$-structure for which the interpretation of the binary function symbol · is an associative operation.) The interpretation of a term $t(\bar{x})$ in a bi-invariant metric group $G$ is then the function $\bar{a} \mapsto t^{G}(\bar{a})$ that replaces the variables $\bar{x}$ with the tuple $\bar{a}$, and then computes the products in $G$. An example of $L$-sentence in this case is given by the $L$-formula $\sup _{x} \sup _{y} d(x \cdot y, y \cdot x)$. The interpretation of such an $L$-sentence $\varphi$ in a bi-invariant metric group is then a real number, which is the supremum of distances of commutators in $G$ from the identity. This can be thought of as a measure of how far $G$ is from being abelian. Clearly, $G$ is abelian if and only if $\varphi^{G}=0$.

We conclude this series of examples by considering the case of the language for $\mathrm{C}^{*}$-algebras $L^{\mathrm{C}^{*}}$. In this case, an $L^{\mathrm{C}^{*}}$-term in the variables $x_{1}, \ldots, x_{n}$ can be seen as a complex polynomial with constant term in the variables $x_{1}, \ldots, x_{n}$ and their "formal adjoint" $x_{1}^{*}, \ldots, x_{n}^{*}$ (*-polynomials). This is strictly speaking not entirely correct, since the term $\left(\left(x_{1}+x_{2}\right)^{*}\right)$, for instance, is not equal to the term $\left(\left(x_{1}\right)^{*}+\left(x_{2}\right)^{*}\right)$. However, they have the same interpretation in every $\mathrm{C}^{*}$-algebra, and hence we can for all purposes identify them, and write them 
simply as $x_{1}^{*}+x_{2}^{*}$. The same applies to the terms $\left(\left(x_{1}+x_{2}\right)+x_{3}\right)$ and $\left(x_{1}+\left(x_{2}+x_{3}\right)\right)$, which we write simply as $x_{1}+x_{2}+x_{3}$. Thus, an atomic $L^{\mathrm{C}^{*}}$-formula in the free variables $x_{1}, \ldots, x_{n}$ can be seen as an expression of the form $\left\|\mathfrak{p}\left(x_{1}, \ldots, x_{n}\right)\right\|$ where $\mathfrak{p}\left(x_{1}, \ldots, x_{n}\right)$ is a ${ }^{*}$-polynomial in the variables $x_{1}, \ldots, x_{n}$. Its interpretation in a $\mathrm{C}^{*}$-algebra $A$ is the function $A^{n} \rightarrow \mathbb{R}, \bar{a} \mapsto \mathfrak{p}(\bar{a})$. An example of $L^{\mathrm{C}^{*}}$-sentence in this setting is, for instance the $L^{\mathrm{C}^{*}}$-formula $\sup _{x \in D_{1}}\left|\left\|x^{*} x\right\|-\|x\|^{2}\right|$. (Recall that in $L^{\mathrm{C}^{*}}$ we have domains of quantification $D_{n}$ for $n \in \mathbb{N}$, which are interpreted in a $\mathrm{C}^{*}$-algebra as the balls of radius $n$ centered at the origin.) Clearly, the interpretation of such an $L^{\mathrm{C}^{*}}$-sentence is equal to 0 in any $\mathrm{C}^{*}$-algebra, in view of the $\mathrm{C}^{*}$-identity.

2.6. Multi-sorted languages. One can consider a further generalization of the framework introduced in Subsection 2.3, by allowing multi-sorted languages. In this setting, the language prescribes a collection $\mathcal{S}$ of sorts. Each sort $S$ in $\mathcal{S}$ comes with a corresponding collection $\mathcal{D}_{S}$ of domains of quantification for $S$. Furthermore, each $n$-ary function and relation symbol has a prescribed $n$-tuple of input sorts and, in the case of function symbols, an output sort as well. A structure $M$ then consists of a family $\left(M^{S}\right)_{S \in \mathcal{S}}$ of metric spaces, one for each sort, together with the corresponding interpretations of domains of quantification, and function and relation symbols, subject to the same requirements as in Subsection 2.3. All the notions and results that we will present admit natural generalizations to the case of multi-sorted languages.

\section{Axiomatizability AND DEFinability}

3.1. Axiomatizable classes. As we have mentioned in Subsection 2, model theory focuses on the study of classes of objects of the same kind, rather than single objects on their own. We have introduced the notion of language, in order to make precise what it means that a class consists objects of the same kind. We have also defined the notion of formula in a given language, which is an expression that allows one to describe properties of an arbitrary tuple of elements of a structure or, in the case of sentences (formulas with no free variables), of the structure itself. This leads to the important notion of elementary or axiomatizable property. By considering the class of structures that satisfy the given property, one can equivalently speak of elementary or axiomatizable class.

To give the precise definition, let us fix a language $L$, and a class of $L$-structure $\mathcal{C}$. Recall that given an $L$-sentence $\varphi$ and a real number $r$, we let $\varphi^{M}$ be the interpretation of $\varphi$ in $M$, which is a real number. An $L$-condition is an expression $\varphi \leq r$ where $\varphi$ is an $L$-sentence and $r \in \mathbb{R}$ is a real number. An $L$-structure $M$ satisfies the condition $\varphi \leq r$ if and only if $\varphi^{M} \leq r$.

Definition 3.1. The class $\mathcal{C}$ is $L$-axiomatizable or $L$-elementary if there exists a family of $L$-conditions $\varphi_{i} \leq r_{i}$ for $i \in I$ such that, given an arbitrary $L$-structure $M$, we have that $M$ belongs to $\mathcal{C}$ if and only if $M$ satisfies the condition $\varphi_{i} \leq r_{i}$ for every $i \in I$. In this case, one refers to the conditions $\varphi_{i} \leq r_{i}$ for $i \in I$ as axioms for $\mathcal{C}$.

One then says that a property $\mathcal{P}$ is $L$-elementary if the class of $L$-structures that satisfy $\mathcal{P}$ is elementary.

It is clear that in the definition of $L$-axiomatizable class, up to replacing each sentence $\varphi_{i}$ with the sentence $\max \left\{\varphi_{i}-r_{i}, 0\right\}$, one can always assume that $r_{i}=0$ and that $\varphi_{i}$ only attains non-negative values. In practice, when the language $L$ is clear from the context, one simply speaks of axiomatizable or elementary class, omitting the explicit reference to the language $L$. Intuitively, the assertion that a property is $L$-elementary means that it can be described by only referring to the elements of a given structure and to the operations of the structure which are named by the language $L$.

In order to gain some familiarity with the concept of axiomatizable class, let us look at examples, drawn from the list of languages and structures that we have considered in Subsection 2. We have introduced above the class of bi-invariant metric groups. For convenience, we can regard these objects as structures in the language $L$ that contains, besides the metric symbol, a binary function symbol · for the operation, a unary function symbol inv for the inverse map, and a constant symbol 1 for the neutral element. Thus the class $\mathcal{C}$ of bi-invariant metric groups forms a class of $L$-structures. This class is easily seen to be axiomatizable, as witnessed by the axiom:

- $\sup _{x} d(x \cdot 1, x) \leq 0$, which prescribes that 1 is interpreted as a neutral element;

- $\sup _{x} d(x \cdot \operatorname{inv}(x), 1) \leq 0$, which prescribes that $\operatorname{inv}(x)$ is interpreted as the inverse of $x$;

- $\sup _{x, y, z} d((x \cdot(y \cdot z)),((x \cdot y) \cdot z)) \leq 0$, which prescribes that the operation is associative;

- $\sup _{x_{0}, x_{1}, y} \max \left\{\left|d\left(x_{0} y, x_{1} y\right)-d\left(x_{0}, x_{1}\right)\right|,\left|d\left(y x_{0}, y x_{1}\right)-d\left(x_{0}, x_{1}\right)\right|\right\} \leq 0$, which forces left and right translations to be isometric.

Several natural properties of bi-invariant metric groups are elementary in this language. For instance, the property of being abelian is elementary, as witnessed by the single axiom $\sup _{x, y} d(x y, y x) \leq 0$. 
Given a collection $\mathcal{C}$ of structures in a language $L$, one of the fundamental problems of the model-theoretic study of $\mathcal{C}$ is understanding which sub-classes of $\mathcal{C}$ (including $\mathcal{C}$ itself) are $L$-axiomatizable. While in some cases this might be apparent, other cases might be more subtle. Often a proof of axiomatizability of a given property might require obtaining an equivalent "explicit" or "quantitative" characterization of such a property, which is easily seen to be captured by $L$-sentences. Finally, there are many natural properties which turn out to be not elementary (although there might be other ways to describe them model-theoretically).

Due to the importance of this task, model theory has developed many useful criteria that can be used in axiomatizability proofs. Often such criteria provide a "softer" approach, which allows one to prove that a certain class is axiomatizable without the need of explicitly write down axioms for it. Here, we will content ourselves to verify that certain classes of structures are axiomatizable by directly applying the definition. In the next section, we will consider the subtle problem of axiomatizability for various important classes of $\mathrm{C}^{*}$-algebras.

3.2. Axiomatizability in $\mathbf{C}^{*}$-algebras. A substantial amount of the recent efforts in the model-theoretic study of $\mathrm{C}^{*}$-algebras has been directed into understanding which classes of $\mathrm{C}^{*}$-algebras are axiomatizable in the canonical language $L^{\mathrm{C}^{*}}$ described in Subsection 2.4, starting from the class of $\mathrm{C}^{*}$-algebras itself. Recall that we are tacitly assuming all $\mathrm{C}^{*}$-algebras to be unital.

3.2.1. $C^{*}$-algebras. A $\mathrm{C}^{*}$-algebra $A$ is a unital Banach algebra with a conjugate-linear involution $a \mapsto a^{*}$ (unital Banach *-algebra) satisfying the $\mathrm{C}^{*}$-algebra identity $\left\|a^{*} a\right\|=\|a\|^{2}$. It is fairly obvious that one can write down sentences that describe that an $L^{\mathrm{C}^{*}}$-structure satisfying such properties. For example, the assertion that the involution is conjugate linear is captured by the family of axioms

$$
\sup _{x \in D_{n}}\left\|(\lambda x)^{*}-\bar{\lambda} x^{*}\right\| \leq 0
$$

where $n \in \mathbb{N}, \lambda$ varies among all complex numbers, and $\bar{\lambda}$ denotes the conjugate of $\lambda$. Similarly, submultiplicativity of multiplication is reflected by the conditions

$$
\sup _{x, y \in D_{n}}(\|x y\|-\|x\|\|y\|) \leq 0
$$

for $n \in \mathbb{N}$, while the $\mathrm{C}^{*}$-identity is captured by the conditions

$$
\sup _{x \in D_{n}}\left|\left\|x^{*} x\right\|-\|x\|^{2}\right| \leq 0
$$

for $n \in \mathbb{N}$.

The only tricky point is that, as we discussed, when we regard a $\mathrm{C}^{*}$-algebra as an $L^{\mathrm{C}^{*}}$-structure, we insist that the domain $D_{n}$ is interpreted as the ball of radius $n$ centered at the origin. Now, in general this need not be true in an arbitrary $L^{\mathrm{C}^{*}}$-structure. Therefore we need to add axioms that enforce this behaviour of the interpretation of domains. For $n \in \mathbb{N}$, we can consider the conditions

$$
\sup _{x \in D_{n}}\|x\| \leq n,
$$

which clearly guarantees that the norm of any element in the interpretation of $D_{n}$ is at most $n$. At this point, we are only missing axioms that guarantee that any element of norm at most $n$ actually belongs to $D_{n}$. This is made sure by the axioms

$$
\sup _{x \in D_{m}} \inf _{y \in D_{n}}(\|x-y\|-\max \{\|x\|-n, 0\}) \leq 0
$$

for $n \leq m$. Indeed, observe that if $x$ is an element of a $\mathrm{C}^{*}$-algebra $A$ which belongs to the ball of radius $m$ centered at the origin, if one actually has that $\|x\| \leq n$, then $x$ can also be seen as an element $y$ of the ball of radius $n$, witnessing that (3) holds. Otherwise if $n<\|x\| \leq m$, then $y:=\frac{n}{\|x\|} x$ is an element of the ball of radius $n$ such that $\|x-y\| \leq\|x\|-n$, again witnessing that 3 holds.

In order to see that these axioms are sufficient to enforce the desired behaviour on the interpretation of the domains of quantification $D_{n}$ for $n \in \mathbb{N}$, suppose that $M$ is an $L^{\mathrm{C}^{*}}$-structure satisfying (2) and (3), as well as the axioms for unital Banach *-algebras satisfying the $\mathrm{C}^{*}$-identity. We claim then that the interpretation $D_{n}^{M}$ is precisely the ball of $M$ of radius $n$ centered at the origin. Indeed, suppose that $x \in M$ is such that $\|x\| \leq n$. Then, by the definition of structure, $\bigcup_{n \in \mathbb{N}} D_{n}^{M}$ is dense in $M$. Hence, for every $\varepsilon>0$ there exists $m \in \mathbb{N}$ and $y \in D_{m}^{M}$ such that $\|x-y\| \leq \varepsilon$. After replacing $y$ with $\frac{y}{1+\varepsilon / n}$ we can assume that $\|y\| \leq n$. Therefore by (3) there exists $z \in D_{n}^{M}$ such that $\|y-z\| \leq \varepsilon$ and hence $\|x-z\| \leq 2 \varepsilon$. Since this holds for every $\varepsilon>0$, since $D_{n}^{M}$ is closed we conclude that $x \in D_{n}^{M}$. Conversely if $x \in D_{n}^{M}$ then $\|x\| \leq n$ by (2). 
In conclusion, we have shown that the class of $\mathrm{C}^{*}$-algebras is axiomatizable in the language $L^{\mathrm{C}^{*}}$ introduced in Subsection 2.4. This paves up the way of establishing similar results for other subclasses that naturally arise in $\mathrm{C}^{*}$-algebra theory. This can be seen as the necessary first step towards the application of methods from logic to $\mathrm{C}^{*}$-algebras.

3.2.2. Abelian $C^{*}$-algebras. The first natural class to consider is the class of abelian $\mathrm{C}^{*}$-algebras, which are the $\mathrm{C}^{*}$-algebras for which the multiplication is commutative. This very definition makes it clear that this class is axiomatizable, by the axiom $\sup _{x, y \in D_{1}}\|x y-y x\| \leq 0$. Abelian $\mathrm{C}^{*}$-algebras are precisely those of the form $C(X)$ for some compact Hausdorff space $X$ (endowed with the pointwise operations and the supremum norm). This motivates the assertion that arbitrary $\mathrm{C}^{*}$-algebras can be regarded as a noncommutative analog of compact Hausdorff spaces, and $\mathrm{C}^{*}$-algebra theory as noncommutative topology.

The class of nonabelian $\mathrm{C}^{*}$-algebras is also axiomatizable, although this is not immediately obvious from the definition. However, this is made it apparent by the following equivalent characterization: a $\mathrm{C}^{*}$-algebra is nonabelian if and only if it contains an element $x$ such that $\|x\|=1$ and $x^{2}=0$ [13, Proposition II.6.4.14]. Thus the class of nonabelian $\mathrm{C}^{*}$-algebra is axiomatizable as witnessed by the condition $\inf _{x \in D_{1}}\left\|x^{2}\right\|-\|x\| \leq-1$.

3.2.3. Nontrivial $C^{*}$-algebras. A $C^{*}$-algebra $A$ is nontrivial if it has dimension at least 2 or, equivalent, $A$ is not isomorphic to $\mathbb{C}$ with its canonical $\mathrm{C}^{*}$-algebra structure. If $A$ is nontrivial, then it contains a selfadjoint element $a \in A$ such that the abelian $\mathrm{C}^{*}$-subalgebra $A_{0}$ of $A$ generated by $a$ and 1 has dimension at least 2 . Thus $A_{0}$ is isomorphic to the algebra $C(X)$ of continuous function over a compact Hausdorff space $X$ with at least 2 points. Hence by normality of $X$ we can find positive elements $b, c \in A_{0}$ such that $\|b\|=\|c\|=\|b-c\|=1$. Recall that any positive element $b$ in a $\mathrm{C}^{*}$-algebra is of the form $a^{*} a$. This shows that the class of nontrivial $\mathrm{C}^{*}$-algebras is axiomatized by the condition

$$
\sup _{x, y \in D_{1}} \min \left\{\|x\|,\|y\|,\left\|x^{*} x-y^{*} y\right\|\right\} \geq 1 .
$$

3.2.4. $n$-subhomogeneous $C^{*}$-algebras. As a generalization of the class of abelian $\mathrm{C}^{*}$-algebras, one can consider the class of $n$-subhomogeneous $\mathrm{C}^{*}$-algebras for some $n \in \mathbb{N}$. Recall that a $\mathrm{C}^{*}$-algebra is $n$-subhomogeneous if and only if all its irreducible representation are $k$-dimensional for some $k \leq n$. When $n=1$, this recovers the class of abelian $\mathrm{C}^{*}$-algebras. It is not obvious by this definition that the class of $n$-subhomogeneous $\mathrm{C}^{*}$-algebras is axiomatizable. Indeed, this definition refers to entities, such as irreducible representations, that are external to the algebra itself. We would rather need an equivalent characterization that only refers to the elements of the algebra and their relations as expressed by the norm and *-algebra operations. Such a characterization can be extracted from a theorem of Amitsur-Levitzki, which isolates an algebraic relation that is satisfied by all the elements of $M_{k}(\mathbb{C})$ for $k \leq n$, but is not satisfied by some elements of $M_{d}(\mathbb{C})$ whenever $d>n$. This relation is given by the expression

$$
\sum_{\sigma \in S_{2 n}} \operatorname{sgn}(\sigma) x_{\sigma(1)} x_{\sigma(2)} \cdots x_{\sigma(2 n)}=0
$$

where $S_{2 n}$ denotes the group of permutations of the set $\{1,2, \ldots, 2 n\}$, and $\operatorname{sgn}(\sigma) \in\{ \pm 1\}$ denotes the parity of the given permutation. This allows one to conclude that a $\mathrm{C}^{*}$-algebra $A$ is $n$-subhomogeneous if and only if any $n$-tuple of elements of $A$ satisfies (4). In order words, the class of $n$-subhomogeneous $\mathrm{C}^{*}$-algebras is axiomatized by the condition

$$
\sup _{x \in D_{1}}\left\|\sum_{\sigma \in S_{2 n}} \operatorname{sgn}(\sigma) x_{\sigma(1)} x_{\sigma(2)} \cdots x_{\sigma(2 n)}\right\| \leq 0 .
$$

A similar argument shows that the class of algebras that are not $n$-subhomogeneous is also axiomatizable.

While the above discussion is reassuring, it turns out that several natural classes of $\mathrm{C}^{*}$-algebras which are key importance in modern $\mathrm{C}^{*}$-algebra theory and in the classification program are not axiomatizable. These include the classes of simple $\mathrm{C}^{*}$-algebras, nuclear $\mathrm{C}^{*}$-algebra, exact $\mathrm{C}^{*}$-algebras, UHF $\mathrm{C}^{*}$-algebras, $\mathrm{AF} \mathrm{C}^{*}$-algebras, and several other. To see why this is the case, we will need to develop a bit more machinery, so we postpone the proof to Subsection 7.2. On the positive side, these classes of algebras can still be captured model-theoretically, although in a slightly more sophisticated way. This will be subject of Subsection 7.4.

3.3. Definable sets. The notion and study of definability is arguably one of the cornerstones of model theory, both in the discrete setting and in the continuous one. In discrete first-order logic, a subset of a structure is definable whenever can be written as the set of elements that satisfy a certain formula. The naive analogue of this definition in the metric setting turns out to be too generous. The right generalization involves the notion, which is unique to the metric setting, of stability of formulas and relations. 
As usual, we begin with a general discussion of stability in logic for metric structures, before specifying the analysis to $\mathrm{C}^{*}$-algebras. Let us therefore fix an arbitrary language $L$ (with domains of quantification), and an elementary class $\mathcal{C}$. (To fix the ideas, one can think of $L$ to be the language of $\mathrm{C}^{*}$-algebras, and $\mathcal{C}$ be the class of all $\mathrm{C}^{*}$-algebras.) Fix also a tuple $\bar{x}=\left(x_{1}, \ldots, x_{n}\right)$ of variables with corresponding domains $\bar{D}=\left(D_{1}, \ldots, D_{n}\right)$, and let $\mathfrak{F}(\bar{x})$ be the collection of $L$-formulas with free variables from $\bar{x}$. Then $\mathfrak{F}(\bar{x})$ admits a natural real Banach algebra structure, induced from the algebra structure on $\mathbb{R}$. For instance, the sum of formulas $\varphi, \psi$ is just the formula $\varphi+\psi$. Furthermore, one can define a seminorm on $\mathfrak{F}(\bar{x})$ by setting

$$
\|\varphi\|=\sup \left\{\varphi^{M}(\bar{a}): M \in \mathcal{C}, \bar{a} \in D_{1}^{M} \times \cdots \times D_{n}^{M}\right\} .
$$

The Hausdorff completion $\mathfrak{M}(\bar{x})$ of $\mathfrak{F}(\bar{x})$ with respect to such a seminorm is then a Banach algebra, whose elements are called definable predicates (in the language $L$ relative to the class $\mathcal{C}$ ). After identifying a formula with the corresponding element of $\mathfrak{M}(\bar{x})$, one can regard formulas as definable predicates. Conversely, definable predicates are precisely the uniform limits of formulas. Given a definable predicate $\varphi$ and a structure $M \in \mathcal{C}$ one can define its interpretation $\varphi^{M}$, which is a function $\varphi^{M}: D_{1}^{M} \times \cdots \times D_{k}^{M} \rightarrow \mathbb{R}$.

As a natural completion of the space of formulas, definable predicates make it easier to develop the theory smoothly. At the same time, definable predicates can for all purposes being replaced by formulas, and vice versa. For instance, it is easy to see that in the definition of axiomatizable class - Definition 3.1 - one can equivalently consider definable predicates rather than sentences. While this is an obvious observation, we state it explicitly due to its importance.

Proposition 3.2. Suppose that $L$ is a language, and $\mathcal{C}$ is a class of $L$-structure. Then $\mathcal{C}$ is L-axiomatizable if and only if there exist a family $\left(\varphi_{i}\right)_{i \in I}$ of definable predicates in the language $L$ with no free variables and a family $\left(r_{i}\right)_{i \in I}$ of real numbers such that, for every $L$-structure $M, M \in \mathcal{C}$ if and only if $\varphi_{i}^{M} \leq r_{i}$ for every $i \in I$.

Among definable predicates, there is a particularly important class: the stable ones.

Definition 3.3. A definable predicate $\varphi(\bar{x})$ is stable if it satisfies the following: for every $\varepsilon>0$ there exists $\delta>0$ such that if $M \in \mathcal{C}$ and $\bar{a} \in D_{1}^{M} \times \cdots \times D_{n}^{M}$ satisfies $|\varphi(\bar{a})|<\delta$, then there exists $\bar{b} \in D_{1}^{M} \times \cdots \times D_{n}^{M}$ such that $d(\bar{a}, \bar{b})<\varepsilon$ and $\varphi(\bar{b})=0$.

The terminology just introduced is consistent with [19], although the term "weakly stable" is used in [36] in lieu of "stable", to match the corresponding notion of "weakly stable relation" from the C*-algebra literature.

Verifying that a given predicate is stable can be a subtle problem, and such stability problems arise frequently in operator algebras, geometric group theory, and metric geometry. Their solution, both in the positive and in the negative, often requires to use or develop deep and interesting theory. At the same time, establishing that a given predicate is indeed stable often has numerous and interesting consequences for the class of structures under consideration. In the theory of operator algebras, a problem closely related to stability is the problem of liftability of relations; see [61].

The notion of stable predicate allows one to introduce the notion of definability. As above, we assume that $\mathcal{C}$ is an elementary class of $L$-structure. Suppose now that $S: M \mapsto S(M)$ is an assignment of closed subspaces $S(M) \subset D_{1}^{M} \times \cdots \times D_{n}^{M}$ to structures $M$ in $\mathcal{C}$.

Definition 3.4. The assignment $S: M \mapsto S(M)$ is a definable set if there exists a stable definable predicate $\varphi$ such that $S(M)$ is the zeroset $Z\left(\varphi^{M}\right)=\left\{\bar{a} \in D_{1}^{M} \times \cdots \times D_{n}^{M}: \varphi^{M}(\bar{a})=0\right\}$.

Among other things, the importance of definable sets lies in the fact that they can be allowed as additional domains of quantification without changing the notion of definable predicate and axiomatizable class. This is the content of the following proposition, established in [11, Theorem 9.17]; see also [36, Theorem 3.2.2].

Proposition 3.5. Suppose that $S: M \mapsto S(M) \subset D_{1}^{M} \times \cdots \times D_{n}^{M}$ is a definable set for the elementary class of $L$-structures $\mathcal{C}$. If $\bar{x}=\left(x_{1}, \ldots, x_{n}\right)$ is a tuple of variables with domains $\left(D_{1}, \ldots, D_{n}\right), \bar{y}=\left(y_{1}, \ldots, y_{k}\right)$ is a tuple of variables with domains $\left(E_{1}, \ldots, E_{k}\right)$, and $\varphi(\bar{x}, \bar{y}) \in \mathfrak{M}(\bar{x}, \bar{y})$ is a definable predicate in the free variables $(\bar{x}, \bar{y})$, then there exists a definable predicate $\psi(\bar{y}) \in \mathfrak{M}(\bar{y})$ such that, for every structure $M$ in $\mathcal{C}$ and $\bar{b} \in E_{1}^{M} \times \cdots \times E_{k}^{M}$,

$$
\psi^{M}(\bar{b})=\inf \left\{\varphi^{M}(\bar{a}, \bar{b}): \bar{a} \in S(M)\right\} .
$$

The same conclusion holds when replacing inf with sup.

One way to think about Proposition 3.5 is that, if $\varphi(\bar{x}, \bar{y})$ is a definable predicate, then the expression

$$
\inf _{\bar{x} \in S} \varphi(\bar{x}, \bar{y})
$$


is also, or more precisely can be identified with, a definable predicate. This gives us much more flexibility in constructing definable predicates, and it will be used in crucial way to show that certain classes of structures are elementary.

At this point, examples of the general notions just introduced are in order. As usual, we being with examples from the theory of bi-invariant metric groups, where it is easier to fix the ideas. We consider the language $L$ for bi-invariant metric groups consisting of function symbols for group operations, beside the metric symbol, and the elementary class $\mathcal{C}$ of bi-invariant metric group. A natural definable predicate, which is in fact a formula, that one can consider in this setting is $\varphi(x, y)=d(x \cdot y, y \cdot x)$. Clearly, such a formula measures how close two elements of a given bi-invariant metric group are to be commuting. Several important problems in operator algebras and metric geometry boils down to the question of whether $\varphi$ is stable for a certain class of bi-invariant metric groups. An important result of Voiculescu shows that $\varphi$ is not stable for the class $\left\{U_{n}: n \in \mathbb{N}\right\}$ of unitary groups endowed with the operator norm [84]. On the other hand, such a formula is stable for the class of unitary groups endowed with the normalized Hilbert-Schmidt norm, as shown by Glebsky [49]. Recently the analogous assertion for the class $\left\{S_{n}: n \in \mathbb{N}\right\}$ of permutation groups with the normalized Hamming distance has been established by Arzhantseva-Paunescu [2]. This important result relies on the Elek-Szabó result on essential uniqueness of sofic representations of amenable groups [28].

3.4. Definability in $\mathbf{C}^{*}$-algebras. Understanding which subsets of $\mathrm{C}^{*}$-algebras are definable is a basic but fundamental problem, that underpins most of the further model-theoretic analysis of $\mathrm{C}^{*}$-algebras. It turns out that, fortunately, several important subsets of $\mathrm{C}^{*}$-algebras are indeed definable. Let us therefore consider the language of $\mathrm{C}^{*}$-algebras $L^{\mathrm{C}^{*}}$ and the class $\mathcal{C}$ of $L^{\mathrm{C}^{*}}$-structures consisting of all $\mathrm{C}^{*}$-algebras. In the following we will consider definable predicates and definable sets with respect to such a class $\mathcal{C}$.

3.4.1. The unitary group. We being by considering the unitary group $U(A)$ of a $\mathrm{C}^{*}$-algebra $A$. This is the set of elements $u$ of $A$ such that $u u^{*}=u^{*} u=1$. Since $U(A)$ is a subset of the unit ball of $A$, the assignment $A \mapsto U(A) \subset D_{1}^{A}$ fits in the framework considered in Subsection 3.3. Naturally, this is the zeroset of the formula $\max \left\{\left\|x x^{*}-1\right\|,\left\|x^{*} x-1\right\|\right\}$. The fact that such a formula is stable can be verified by using the polar decomposition of operators [64, Theorem 3.2.17]. Indeed, if $A \subset B(H)$ is a $\mathrm{C}^{*}$-algebra, and $a \in A$, then one can write $a=u\left(a^{*} a\right)^{1 / 2}$ where $u \in B(H), u^{*} u$ is the orthogonal projection onto the orthogonal complement of the kernel of $\left(a^{*} a\right)^{1 / 2}$, and $u u^{*}$ is the orthogonal projection onto the orthogonal complement of the kernel of $\left(a a^{*}\right)^{1 / 2}$. Now, if $\left\|a^{*} a-1\right\| \leq \delta$ and $\left\|a a^{*}-1\right\| \leq \delta$ for some small enough $\delta,\left(a^{*} a\right)^{1 / 2}$ and $\left(a a^{*}\right)^{1 / 2}$ are invertible, which forces $u$ to be a unitary and to belong to $A$. Finally,

$$
\|u-a\| \leq\left\|1-\left(a^{*} a\right)^{1 / 2}\right\|
$$

which can be made arbitrarily small by choosing $\delta$ small enough. A similar argument shows that the set of isometries (i.e. elements $v$ of $A$ satisfying $v^{*} v=1$ ) is also definable.

3.4.2. Positive contractions. A similar argument allows one to conclude that the set $A_{+}^{1}$ of positive contractions in $A$ is a definable set. Recall that an element $a$ of $A$ is positive if it is a positive operator in any faithful representation $A \subset B(H)$. This is equivalent to the assertion that $a$ is of the form $b^{*} b$ for some $b \in A$. A positive contraction is just an positive element of norm at most 1 . Therefore the set of positive contraction is the zeroset of the formula $\inf _{y \in D^{1}}\left\|x-y^{*} y\right\|$, which is obviously stable. In a similar way, considering the stable formula $\left\|x^{*}-x\right\|$, shows that the set $A_{\mathrm{sa}}^{1}$ of selfadjoint elements of norm at most 1 is definable as well.

3.4.3. Projections. We now consider the set of projections in a $\mathrm{C}^{*}$-algebra $A$. These are the selfadjoint elements $p$ of $A$ satisfying $p^{2}=p$. By definition, this is the zeroset of the formula $\varphi(x)$ given by $\max \left\{\left\|x^{*}-x\right\|,\left\|x^{2}-x\right\|\right\}$. To see that such a formula is stable, suppose that $a \in A$ is an element of norm at most 1 satisfying $\left\|a^{*}-a\right\| \leq \delta$ and $\left\|a^{2}-a\right\| \leq \delta$ for some $\delta \in(0,1 / 2)$. Consider $a_{0}:=\left(a+a^{*}\right) / 2$ and observe that $\left\|a_{0}\right\| \leq 1,\left\|a-a_{0}\right\|<\bar{\delta} / 2$ and hence $\left\|a_{0}^{2}-a_{0}\right\|<2 \delta$. This implies that the spectrum of $a_{0}$ is contained in $(-\varepsilon, \varepsilon) \cup(1-\varepsilon, 1]$ where $\varepsilon=\frac{1-\sqrt{1-8 \delta}}{2} \leq \delta$. Therefore the function

$$
f(t)=\left\{\begin{array}{cc}
0 & \text { if } t \in(-\delta, \delta), \\
1 & \text { if } t \in(1-\delta, 1]
\end{array}\right.
$$

is continuous on the spectrum of $a$. By continuous functional calculus, one can consider the element $p:=f\left(a_{1}\right)$ of $A$. Since the spectrum of $p$ is the range of $f$, it is contained in $\{0,1\}$, which implies that $p$ is a projection. Furthermore $\left\|p-a_{1}\right\|$ is equal to the supremum of $|f(t)-t|$ where $t$ ranges in the spectrum of $a$, which is at most $\delta$. In conclusion $\left\|p-a_{0}\right\| \leq\left\|p-a_{1}\right\|+\left\|a_{1}-a_{0}\right\|<\delta+2 \delta \leq 3 \delta$. In conclusion, this shows that if $a$ is an 
element of the unit ball of a $\mathrm{C}^{*}$-algebra $A$ satisfying $\varphi^{A}(a)<\delta$, then there exists $p$ in the unit ball of $A$ such that $\varphi^{A}(p)=0$ and $\|p-a\|<3 \delta$.

In a similar fashion, one can show that the set of $n$-tuples $\left(p_{1}, \ldots, p_{n}\right)$ of pairwise orthogonal projections of $A$ is definable. (Two projections $p, q$ are orthogonal if $p q=q p=0$.) Let us consider for simplicity the case $n=2$. Let $\varphi(x, y)$ be the formula

$$
\max \left\{\left\|x^{*}-x\right\|,\left\|x^{2}-x\right\|,\left\|y-y^{*}\right\|,\left\|y^{2}-y\right\|,\|x y\|,\|y x\|\right\} .
$$

Clearly, the zeroset of $\varphi$ is the set of pairs of orthogonal projections. In order to see that $\varphi$ is stable, fix $\varepsilon>0$, and suppose that $a, b$ are elements of the unit ball of a $\mathrm{C}^{*}$-algebra $A$ satisfying $\varphi^{A}(a, b)<\delta<\varepsilon / 2$ for some small enough $\delta$. By stability of the formula defining projections, we can assume that $a, b$ are already projections. Then one can set $p=a$ and consider $b_{0}:=(1-p) b(1-p) \in(1-p) A(1-p)$. Since $\|a b\|<\delta$ and $\|b a\|<\delta$ one has that $\left\|b_{0}-b\right\|<2 \delta$. Again by stability of the formula defining projections applied to the $\mathrm{C}^{*}$-algebra $(1-p) A(1-p)$, for $\delta$ small enough there exists a projection $q \in(1-p) A(1-p)$ such that $\left\|q-b_{0}\right\|<\varepsilon / 2$ and hence $\|q-b\|<\varepsilon$. Observing that $q \in A$ is orthogonal to $p$ concludes the proof that $\varphi$ is stable.

3.4.4. Partial isometries. Recall that a partial isometry in a $\mathrm{C}^{*}$-algebra $A$ is an element $v \in A$ such that $v^{*} v$ is a projection, called the support projection of $v$. This implies that $v v^{*}$ is also a projection, called the range projection of $v$. We want to show that the set of partial isometries is definable. The following lemma can be extracted from the classical paper of Glimm classifying UHF algebras; see [50, Lemma 1.9].

Lemma 3.6. Suppose that $\delta \in(0,1 / 40)$ and $A$ is a $\mathrm{C}^{*}$-algebra. Fix a concrete representation $A \subset B(H)$ of $A$ as an algebra of operators on a Hilbert space $H$. Suppose that $p, q \in A$ are projections, and $\tilde{p}, \tilde{q} \in B(H)$ are projections such that $\|p-\tilde{p}\|<\delta,\|q-\tilde{q}\|<\delta$. Assume that there exist a partial isometry $\tilde{v} \in B(H)$ and $a \in A$ such that $\tilde{v}^{*} \tilde{v}=\tilde{p}, \tilde{v} \tilde{v}^{*}=\tilde{q},\|a-\tilde{v}\|<\delta$, and $\|a\| \leq 1$. Then there exists a partial isometry $v \in A$ such that $v^{*} v=p, v v^{*}=q$, and $\|v-\tilde{v}\|<30 \delta$.

We can use this lemma to show that the set of partial isometries of $A$ is definable. The set of partial isometries is the zeroset of the formula $\max \left\{\left\|x^{*} x-\left(x^{*} x\right)^{2}\right\|,\left\|x x^{*}-\left(x x^{*}\right)^{2}\right\|\right\}$. We claim that such a formula is stable. Suppose that $a \in A \subset B(H)$ satisfies $\left\|a^{*} a-\left(a^{*} a\right)^{2}\right\| \leq \delta \leq 1 / 1600$. By functional calculus this implies that $\left\|\left(a^{*} a\right)^{1 / 2}-\left(a^{*} a\right)\right\| \leq \delta$. Using the polar decomposition of operators, we can write $a=\tilde{v}\left(a^{*} a\right)^{1 / 2}$, where $\tilde{v} \in$ $B(H)$ is a partial isometry with support projection the orthogonal projection $\tilde{p}$ onto $\operatorname{Ker}(a)^{\perp}=\operatorname{Ker}\left(a^{*} a\right)^{\perp}=$ $\overline{\operatorname{Ran}\left(a^{*} a\right)}$ and range projection the orthogonal projection onto $\operatorname{Ker}\left(a^{*}\right)^{\perp}=\operatorname{Ker}\left(a a^{*}\right)^{\perp}=\overline{\operatorname{Ran}\left(a a^{*}\right)}$. (For an operator $T \in B(H)$ we let $\operatorname{Ker}(T)$ be the kernel $\{\xi \in H: T \xi=0\}$ of $T$, and $\overline{\operatorname{Ran}(T)}$ the closure of the range $\{T \xi: \xi \in H\}$ of $T$.) Now for $\xi \in \operatorname{Ran}\left(a^{*} a\right)$ we have that $\xi=\left(a^{*} a\right) \eta$ for some $\eta \in H$ and hence

$$
\left\|\left(a^{*} a\right) \xi-\xi\right\|=\left\|\left(a^{*} a\right)^{2} \eta-\left(a^{*} a\right) \eta\right\| \leq\left\|\left(a^{*} a\right)^{2}-\left(a^{*} a\right)\right\| \leq \delta \text {. }
$$

This shows that $\left\|a^{*} a-\tilde{p}\right\| \leq \delta$. Similarly, one has that $\left\|a a^{*}-\tilde{q}\right\| \leq \delta$. Thus

$$
\|a-\tilde{v}\|=\left\|\tilde{v}\left(a^{*} a\right)^{1 / 2}-\tilde{v}\right\| \leq\left\|\left(a^{*} a\right)^{1 / 2}-p\right\| \leq \sqrt{\delta} .
$$

Furthermore by stability of formula defining projections we have that there exist projections $p, q \in A$ such that $\left\|p-\left(a^{*} a\right)^{1 / 2}\right\| \leq 3 \delta$ and $\left\|q-\left(a a^{*}\right)^{1 / 2}\right\| \leq 3 \delta$. Therefore $\|p-\tilde{p}\| \leq 4 \delta \leq \sqrt{\delta}$ and $\|q-\tilde{q}\| \leq 4 \delta \leq \sqrt{\delta}$. Therefore by Lemma there exists a partial isometry $v \in A$ with support projection $p$ and range projection $q$ such that $\|v-\tilde{v}\| \leq 30 \sqrt{\delta}$.

3.4.5. Murray-von Neumann equivalence. Murray-von Neumann equivalence is an important relation among projections in a $\mathrm{C}^{*}$-algebra, which is crucial for the definition of the $K_{0}$-group. Recall that an element $v$ of a $\mathrm{C}^{*}$-algebra $A$ is a partial isometry if $v^{*} v$ is a projection (support projection), and $v v^{*}$ is a projection (range projection). Two projections $p, q \in A$ are Murray-von Neumann equivalent -in formulas $p \sim q$-if there exists a partial isometry $v \in A$ such that $p$ is the support projection of $v$ and $q$ is the range projection of $v$. We recall that if $p, q \in A$ are projections such that $\|p-q\|<1$ then $p \sim q$.

We want to show that the relation of Murray-von Neumann equivalence of projections (as a set of pairs) is definable. Indeed, consider the definable predicate $\varphi(x, y)$ given by

$$
\max \left\{\left\|x^{2}-x\right\|,\left\|x-x^{*}\right\|,\left\|y^{2}-y\right\|,\left\|y-y^{*}\right\|, \underset{z \text { partial isometry }}{\max }\left\{\left\|z^{*} z-x\right\|,\left\|z z^{*}-y\right\|\right\}\right\} .
$$


Then we claim that the zeroset of $\varphi$ in a $\mathrm{C}^{*}$-algebra $A$ is the relation of Murray-von Neumann equivalence of projections. Indeed, if $\varphi^{A}(p, q)=0$ then clearly $p, q$ are projections. Furthermore, there exists a partial isometry $v \in A$ such that $\left\|v^{*} v-p\right\|<1$ and $\left\|v v^{*}-q\right\|<1$. This implies $p \sim v^{*} v \sim v v^{*} \sim q$. It follows easily from stability of the formula defining projections that $\varphi$ is stable as well. This shows that the relation of Murray-von Neumann equivalence of projections is a definable set. Adding the clauses $|\|x\|-1|$ and |||y||-1| shows that the relation of Murray-von Neumann equivalence of nonzero projections is a definable set. A similar argument shows that the set of $n$-tuples of pairwise orthogonal and pairwise Murray-von Neumann equivalent projections is definable.

3.4.6. Infinite projections. Suppose that $A$ is a $\mathrm{C}^{*}$-algebra. Recall that for projections $p, q \in A$ one sets $p \leq q$ if $p q=q p=p$. Observe that if $p \leq q$ and $p \neq q$, then $\|p-q\|=1$. A nonzero projection $r \in A$ is called

- infinite if there is a nonzero projection $r_{0} \leq r$ such that $r_{0} \neq r$ and $r_{0} \sim r$,

- properly infinite if there exist nonzero orthogonal Murray-von Neumann equivalent projections $p, q \in A$ such that $p+q=r$.

We claim that the set of infinite projections in a $\mathrm{C}^{*}$-algebra is definable. As we have observed above, there is a definable predicate $\psi(x, y)$ whose zeroset is the relation of Murray-von Neumann equivalence of nonzero projections. We can therefore consider the definable predicate $\theta(x)$ given by

$$
\inf _{y \in D_{1}} \max \{\psi(x, y),\|y x-y\|,\|x y-y\|,|1-\|x-y\||\} .
$$

It is clear by the above remarks that the set of infinite projections is contained in the zeroset of $R$. In order to show that $\theta$ is a stable definable predicate whose zeroset is the set of infinite projections it remains to show the following: for every $\varepsilon>0$ there exists $\delta>0$ such that for every $\mathrm{C}^{*}$-algebra $A$ and $p \in A$ satisfying $\theta^{A}(p)<\delta$ there exists an infinite projection $p^{\prime} \in A$ such that $\left\|p-p^{\prime}\right\|<\varepsilon$. Let us thus consider $p \in A$ such that $\theta^{A}(p)<\delta$. By stability of the predicate $p$, we can assume that $p$ is itself a nonzero projection. Furthermore, by stability of the predicate $Q$, we can assume, up to replacing $\delta$ with a smaller positive number, that there exists a nonzero projection $q \in A$ such that $q \sim p,\|p q-q\|<\delta,\|q p-q\|<\delta$, and $|1-\|p-q\||<\delta$. Consider now $p q p \in p A p$ and observe that, again by stability of the predicate defining projections, and upon replacing $\delta$ with a smaller positive number, we can find a projection $q^{\prime} \in p A p$ such that $\left\|q^{\prime}-q\right\|<\delta$. This guarantees that $p q^{\prime}=q^{\prime} p=q^{\prime}$ and $\left|1-\left\|p-q^{\prime}\right\|\right|<2 \delta$. As long as $\delta<1 / 2$ this ensures that $q^{\prime}$ is a nonzero projection such that $q^{\prime} \leq p, q^{\prime} \neq p$, and $q^{\prime} \sim q \sim p$. Therefore $p$ is itself infinite projection, concluding the proof that $\theta$ is stable.

We now claim that the set of properly infinite projections in a $\mathrm{C}^{*}$-algebra is definable. Indeed, let now $\varphi(x)$ be the definable predicate whose zeroset is the set of nonzero projections. Then we can consider, in view of definability of the relation of Murray-von Neumann equivalence of orthogonal nonzero projections, the definable predicate $\eta(x)$ given by

$$
\max \left\{\varphi(x), \inf _{y, z \text { nonozero orthogonal projections, } y \sim z}\|x-(y+z)\|\right\} .
$$

As above, it is clear that the set of properly infinite projections is contained in the zeroset of $S$. In order to show that $\eta$ is a stable definable predicate whose zeroset is the set of properly infinite projections, it remains to show the following: for every $\varepsilon>0$ there exists $\delta>0$ such that if $A$ is a $\mathrm{C}^{*}$-algebra and $r \in A$ is such that $\eta^{A}(r)<\delta$ then there exists a properly infinite projection $r^{\prime} \in A$ such that $\left\|r-r^{\prime}\right\|<\varepsilon$. Suppose then that $r \in A$ satisfies $\eta^{A}(r)<\delta$. Again, by stability of $\varphi$ we can suppose that $r$ is itself a nonzero projection. Furthermore there exist orthogonal nonzero projections $p, q \in A$ such that $\|r-(p+q)\|<\delta$. Thus $r^{\prime}:=p+q$ is a nonzero purely infinite projection such that $\left\|r-r^{\prime}\right\|<\delta$, concluding the proof.

3.4.7. Scalars. Recall that we are tacitly assuming $\mathrm{C}^{*}$-algebras to be unital. Thus, after identifying a complex number with the corresponding scalar multiple of the unit, we can identify $\mathbb{C}$ with a subalgebra of any given $\mathrm{C}^{*}$-algebra. Below we let $x$ be a variable with corresponding domain $D_{1}$. Then we have that $[0,1]$ is definable, as the zeroset of the stable formula $\|x-\| x\|1\|$. Using this fact and Proposition 3.5, one can conclude that $\inf _{t_{0}, t_{1} \in[0,1]}\left\|x-\left(t_{0}-t_{1}\right)\right\|$ is also a definable predicate, which is obviously stable. Its zeroset is $[-1,1]$. Analogously, the set $\mathbb{D}=\{\lambda \in \mathbb{C}:|\lambda| \leq 1\}$ is the zeroset of the definable predicate $\inf _{t_{0}, t_{1} \in[-1,1]}\left\|x-\left(t_{0}+i t_{1}\right)\right\|$, and the set $\mathbb{T}=\{\lambda \in \mathbb{C}:|\lambda|=1\}$ is the zeroset of the definable predicate $\max \left\{\left\|x^{*} x-1\right\|,\left\|x x^{*}-1\right\|, \inf _{t_{0}, t_{1} \in[-1,1]}\left\|x-\left(t_{0}+i t_{1}\right)\right\|\right\}$. Thus all these sets are definable. 
3.5. More axiomatizable classes of $\mathbf{C}^{*}$-algebras. At this point, we will use the possibility of quantifying over definable sets guaranteed by Proposition 3.5 to show that several other important classes of $\mathrm{C}^{*}$-algebras are axiomatizable.

3.5.1. Stably finite $C^{*}$-algebra. Suppose that $A$ is a $C^{*}$-algebra. An isometry $v$ in $A$ is an element of $A$ satisfying $v^{*} v=1$. A C $\mathrm{C}^{*}$-algebra $A$ is finite if every isometry is a unitary, and stably finite if $M_{n}(A)$ is finite for every $n \in \mathbb{N}$. A similar proof as in the case of partial isometries - see $\S 3.4 .4$-shows that the set of isometries is definable. Therefore the class of finite $\mathrm{C}^{*}$-algebra is elementary, as witnessed by the axiom

$$
\sup _{v \text { isometry }}\left\|v v^{*}-1\right\| \leq 0 .
$$

Since the language of $\mathrm{C}^{*}$-algebras contains relation symbols for the norm in $M_{n}(A)$ for $n \in \mathbb{N}$, one can similarly conclude that the set of isometries in $M_{n}(A)$ is definable. Henceforth, the same argument shows that the class of stably finite $\mathrm{C}^{*}$-algebras is axiomatizable as well.

3.5.2. Real rank zero $C^{*}$-algebras. By definition, a $\mathrm{C}^{*}$-algebra has real rank zero if the set of selfadjoint elements with finite spectrum is dense in the set of all selfadjoint elements. While this definition does not make it apparent that this is a definable property, we can consider the following useful equivalent characterization. A $\mathrm{C}^{*}$-algebra has real rank zero if and only if for every pair of positive elements $a, b$ in $A$ of norm at most 1 and every $\varepsilon>0$ there exists a projection $p \in A$ such that $\max \{\|p a\|,\|(1-p) b\|\}<\|a b\|^{1 / 2}+\varepsilon[17$, Theorem 2.6]. In other words, a $\mathrm{C}^{*}$-algebra has real rank zero if and only if it satisfies the condition

$$
\sup _{\substack{x, y \in D_{1} \\ x, y \text { positive }}} \inf _{\substack{z \in D_{1} \\ \text { projection }}} \max \{\|z x\|,\|(1-z) y\|\}-\|x y\|^{1 / 2} \leq 0 .
$$

By Proposition 3.5 and the fact that the sets of projections and positive contractions are definable, this witnesses that real rank zero $\mathrm{C}^{*}$-algebras form an axiomatizable class.

3.5.3. Purely infinite simple $C^{*}$-algebras. The notion of purely infinite $\mathrm{C}^{*}$-algebra can be defined in terms of the notion of Cuntz equivalence of positive elements. This is an equivalence relation for positive elements in a $\mathrm{C}^{*}$-algebra, generalizing the relation of Murray-von Neumann equivalence for projections. Suppose that $A$ is a $\mathrm{C}^{*}$-algebra, and $a, b$ are positive elements of $A$. Then one sets $a \precsim b$ if there exists a sequence $\left(x_{n}\right)$ in $A$ such that $x_{n}^{*} b x_{n} \rightarrow a$ for $n \rightarrow+\infty$. Then $a$ and $b$ are Cuntz equivalent, in formulas $a \sim b$, if $a \precsim b$ and $b \precsim a$. It can be shown that this is indeed an equivalence relation which, in the case of projections, coincides with Murray-von Neumann equivalence.

For a selfadjoint element $a$ of a $\mathrm{C}^{*}$-algebra $A$ we let $a_{+}$be the positive part of $A$. In other words, $a_{+}$is the element $f(a)$ of $A$ where $f: \mathbb{R} \rightarrow \mathbb{R}$ is the function

$$
f(t)=\left\{\begin{array}{lc}
0 & \text { if } t \leq 0 \\
t & \text { otherwise }
\end{array}\right.
$$

The following lemma is proved in [60, Lemma 2.4].

Lemma 3.7. If $a, b$ are positive elements of a $\mathrm{C}^{*}$-algebra $A$ of norm at most 1 , and $n \in \mathbb{N}$ is such that $a \precsim\left(b-\frac{1}{n^{2}}\right)_{+}$then there exists $c \in A$ such that $\|c\| \leq 2 n$ and $a=c^{*} b c$.

A nontrivial $\mathrm{C}^{*}$-algebra $A$ is purely infinite if it has no nontrivial abelian quotients, and whenever $a, b$ are nonzero positive elements of $A$ such that $b$ belongs to the closed two-sided ideal generated by $a$, then $a \precsim b[70$, Proposition 4.1.1]. A $\mathrm{C}^{*}$-algebra is simple if it contains no nontrivial closed two-sided ideals. For simple $\mathrm{C}^{*}$ algebras, being purely infinite is equivalent to the assertion that $A$ is nontrivial, and whenever $a, b$ are nonzero positive elements of $A, a \sim b$. Furthermore, for nontrivial simple $\mathrm{C}^{*}$-algebras being purely infinite is equivalent to the assertion that $A$ has real rank zero, and any nonzero projection in $A$ is properly infinite.

We have already seen that the class of real rank zero $\mathrm{C}^{*}$-algebras is axiomatizable. Furthermore, the sets of nonzero projections and of properly infinite projections are both definable, which easily implies that the class of $\mathrm{C}^{*}$-algebras with the property that any nonzero projection is properly infinite, is axiomatizable. Let now $\mathcal{C}$ be the axiomatizable class of real rank $\mathrm{C}^{*}$-algebras with the property that any nonzero projection is properly infinite. In view of the remarks above, for a $\mathrm{C}^{*}$-algebra $A$ in $\mathcal{C}$ being simple (and hence purely infinite) is equivalent to the assertion that any two nonzero positive elements of $A$ are Cuntz equivalent. Since any nonzero positive element $a$ of $A$ is Cuntz equivalent to any nonzero positive scalar multiple of $A$, this is in turn equivalent to the assertion that for any two positive elements $a, b$ of $A$ satisfying $\|a\|>1 / 2$ and $\|b\|>1 / 2$ one has that $a \sim b$. By Lemma 3.7 applied in the case when $n=2$ this is in turn equivalence to the assertion that if $a, b$ 
are positive elements of $A$ then for every $\varepsilon>0$ there exists $c \in A$ such that $\|c\| \leq 4$ and $\left\|a-c^{*} b c\right\|<\varepsilon$. This condition is clearly axiomatized by the condition

$$
\sup _{x, y \in D_{1}} \min \left\{\|x\|-1 / 2,\|y\|-1 / 2, \inf _{z \in D_{1}}\left\|a-(4 z)^{*} b(4 z)\right\|\right\} \leq 0 .
$$

This shows that the class of purely infinite simple $\mathrm{C}^{*}$-algebras is axiomatizable.

\section{Ultraproducts AND ULTRAPOWERS}

4.1. Ultraproducts in the logic for metric structures. Ultraproducts and ultrapowers are a fundamental construction in model theory, both in its discrete version and its generalization for metric structures. We introduce this construction in the general setting of an arbitrary language $L$. We work in the setting of languages with domains of quantification introduced in Subsection 2.3.

Let then $I$ be a set, to be considered as an index set. (The reader can consider the case when $I=\mathbb{N}$, for simplicity.) An ultrafilter $\mathcal{U}$ over $I$ is a nonempty collection of subsets of $I$ with the property that $\varnothing \notin \mathcal{U}$, if $A, B \in \mathcal{U}$ then $A \cap B \in \mathcal{U}$, and for every $A \in I$ either $A \in \mathcal{U}$ or $I \backslash A \in \mathcal{U}$. One should consider $\mathcal{U}$ as a notion of largeness, where a set $A \subset I$ is large if it belongs to $\mathcal{U}$. In the spirit of this interpretation, $\mathcal{U}$ can be thought of as a finitely-additive $\{0,1\}$-valued measure on $I$ which is defined for arbitrary subsets of $A$ by

$$
A \mapsto \begin{cases}1 & \text { if } A \in \mathcal{U} \\ 0 & \text { if } A \notin \mathcal{U}\end{cases}
$$

Conversely, any finitely-additive $\{0,1\}$-valued measure on $I$ which is defined on all subsets of $I$ arises from an ultrafilter in this fashion. An ultrafilter $\mathcal{U}$ over $I$ is principal if it contains a finite set (whence it contains a singleton), and nonprincipal otherwise. A stronger properly than being nonprincipal is being countably incomplete, which means that it contains a sequence $\left(X_{n}\right)$ of elements with empty intersection. When $I$ is countable, these two notions coincide.

Fix an ultrafilter $\mathcal{U}$ over $I$. Consistently with the interpretation of ultrafilters as notions of largeness, following [79] we introduce the notation of ultrafilter quantifiers. Let $P$ be a property that elements of $I$ may or may not have. Then we write $(\mathcal{U} i) P(i)$ if the set of elements $i$ of $I$ for which $P$ holds belongs to $\mathcal{U}$.

Suppose now that $f$ is a continuous function $f$ from $I$ to a compact Hausdorff space $X$. One can then define

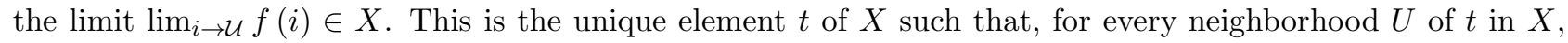
$(\mathcal{U} i) f(i) \in U$. It is clear that, since $X$ is Hausdorff, there exists at most one such an element of $X$. In order to see that such an element of $X$ exists, consider the collection $\mathcal{F}$ of nonempty closed subsets of the form

$$
\overline{\{f(i): i \in A\}}
$$

for $A \in \mathcal{U}$. Then $\mathcal{F}$ satisfies the finite intersection property, and by compactness of $X$ one has that $\bigcap \mathcal{F}$ is nonempty. If $x$ is an element of $\bigcap \mathcal{F}$ and $U$ is an open neighborhood of $x$, then we claim that $(\mathcal{U} i) f(i) \in U$. Indeed, if this is not the case, then $(\mathcal{U} i) f(i) \notin U$. Thus there exists $A \in \mathcal{U}$ such that $\overline{\{f(i): i \in A\}} \subset X \backslash U$. Since $\overline{\{f(i): i \in A\}} \in \mathcal{F}$, this contradicts the fact that $x \in \cap \mathcal{F}$.

Suppose now that $\left(M_{i}\right)_{i \in I}$ is a family of $L$-structures indexed by $I$. The ultraproduct $\prod_{\mathcal{U}} M_{i}$ is the $L$ structure $M$ defined as follows. For every domain $D$ in the language $L$, consider the product $\prod_{i \in I} D^{M_{i}}$. This is naturally endowed with a pseudometric given by $d(\boldsymbol{a}, \boldsymbol{b})=\lim _{i \rightarrow \mathcal{U}} d\left(a_{i}, b_{i}\right)$. Here and in the following, we denote by $\boldsymbol{a}$ an $I$-sequence $\left(a_{i}\right)_{i \in I}$ with $a_{i} \in M_{i}$ for $i \in I$. Then one can define $M^{D}$ to be the metric space obtained from such a pseudometric. If $\boldsymbol{a}$ is an element of $\prod_{i \in I} D^{M_{i}}$, then we let $[\boldsymbol{a}]$ be the corresponding element of $D^{M}$. If $D_{0}, D_{1}$ are domains such that $D_{0} \leq D_{1}$, then by definition of structure one has that $D_{0}^{M_{i}} \subset D_{1}^{M_{i}}$ for every $i \in I$. Thus one can canonically identify isometrically $D_{0}^{M}$ with a subspace of $D_{1}^{M}$. Since the collection $\mathcal{D}$ of domains is directed, the union $\bigcup_{D \in \mathcal{D}} D^{M}$ is itself a metric space, and we let $M$ to be the completion of such a metric space.

We now define the interpretation of function symbols in $M$. Suppose that $f$ is an $n$-ary function symbol in $L$ and $D_{1}, \ldots, D_{n}$ are domains. Then one can consider the corresponding output domain $D=D_{D_{1}, \ldots, D_{n}}^{f}$ and the continuity modulus $\varpi=\varpi_{D_{1}, \ldots, D_{n}}^{f}$ as prescribed by the language $L$. Then, for every $i \in I$, the interpretation of $f$ in $M_{i}$ gives a function $f^{M_{i}}: D_{1}^{M_{i}} \times \cdots \times D_{n}^{M_{i}} \rightarrow D^{M_{i}}$ which is uniformly continuous with modulus $\varpi$. Therefore one can define $f^{M}: D_{1}^{M} \times \cdots \times D_{n}^{M} \rightarrow D^{M}$ by setting

$$
f^{M}\left(\left[\boldsymbol{a}^{(1)}\right], \ldots,\left[\boldsymbol{a}^{(n)}\right]\right)=\left[\left(f^{M_{i}}\left(a_{i}^{(1)}, \ldots, a_{i}^{(n)}\right)\right)_{i \in I}\right] .
$$


This is again a uniformly continuous function with modulus $\varpi$. Letting $D_{1}, \ldots, D_{n}$ range among all the domains in the language $L$ one can then define, by extending it to the completion, the function $f^{M}: M^{n} \rightarrow M$, which is still uniformly continuous with modulus $\varpi$.

Suppose now that $R$ is an $n$-ary relation symbol in $L$ and $D_{1}, \ldots, D_{n}$ are domains. Then one can consider the corresponding output domain $D=D_{D_{1}, \ldots, D_{n}}^{f}$, the continuity modulus $\varpi=\varpi_{D_{1}, \ldots, D_{n}}^{f}$, and the bound $J=J_{D_{1}, \ldots, D_{n}}^{R}$ as prescribed by the language $L$. Then for every $i \in I$, the interpretation of $R$ in $M_{i}$ gives a function $R^{M_{i}}: D_{1}^{M_{i}} \times \cdots \times D_{n}^{M_{i}} \rightarrow J$ which is uniformly continuous with modulus $\varpi$. Therefore one can define $R^{M}: D_{1}^{M} \times \cdots \times D_{n}^{M} \rightarrow J$ by setting

$$
R^{M}\left(\left[\boldsymbol{a}^{(1)}\right], \ldots,\left[\boldsymbol{a}^{(n)}\right]\right)=\lim _{i \rightarrow \mathcal{U}} R^{M_{i}}\left(a_{i}^{(1)}, \ldots, a_{i}^{(n)}\right) \in J
$$

This is again a uniformly continuous function with modulus $\varpi$. Letting $D_{1}, \ldots, D_{n}$ range among all the domains in the language $L$ one can then define, by extending it to the completion, the function $R^{M}: M^{n} \rightarrow \mathbb{R}$, which is still uniformly continuous with modulus $\varpi$.

4.2. Łos' theorem. Łos' theorem is the fundamental result in model theory that relates the construction of ultraproducts with notion of formulas. Let us adopt the notation of the previous section.

Assume that $t$ is an $L$-term with variables within $x_{1}, \ldots, x_{n}$ which have $D_{1}, \ldots, D_{n}$ as corresponding domains. Then one can easily see by induction on the complexity of $t$ that one can define an output domain $D=D_{D_{1}, \ldots, D_{n}}^{t}$ and a continuity modulus $\varpi=\varpi_{D_{1}, \ldots, D_{n}}^{t}$ in terms of the output domains and continuity moduli of the function symbols in $L$, such that for any $L$-structure $N$ the interpretation $t^{N}$ of $t$ in $N$ is a function $t^{N}: D_{1}^{N} \times \cdots \times D_{n}^{N} \rightarrow$ $D^{N}$ with continuity modulus $\varpi$. In particular, this guarantees that, if $M_{\mathcal{U}}$ is the ultraproduct $\prod_{\mathcal{U}} M_{i}$, then the function $D_{1}^{M_{\mathcal{U}}} \times \cdots \times D_{n}^{M_{\mathcal{U}}} \rightarrow D^{M_{\mathcal{U}}}$,

$$
\left(\left[\boldsymbol{a}^{(1)}\right], \ldots,\left[\boldsymbol{a}^{(n)}\right]\right) \mapsto\left[t^{M_{i}}\left(a_{i}^{(1)}, \ldots, a_{i}^{(n)}\right)\right]
$$

is a well-defined function with continuity modulus $\varpi$. Furthermore, it is also easy to show by induction on the complexity of the term $t$, and using the definition of the interpretation of function symbols in $M_{\mathcal{U}}$, that such a function coincides with the interpretation $t^{M_{\mathcal{U}}}$ of the term $t$ in $M_{\mathcal{U}}$.

Suppose now that $\varphi$ is an $L$-formula with free variables within $x_{1}, \ldots, x_{n}$. Again, one can show by induction on the complexity of $\varphi$ that one can define a bound $J=J_{D_{1}, \ldots, D_{n}}^{\varphi}$ and a continuity modulus $\varpi=\varpi_{D_{1}, \ldots, D_{n}}^{\varphi}-$ in terms of the bounds, output domains, and continuity moduli of the terms, connectives, and relation symbols that appear in $\varphi$-such that for any $L$-structure $M$ the interpretation $\varphi^{M}$ of $\varphi$ in $M$ is a function $\varphi^{M}$ : $D_{1}^{M} \times \cdots \times D_{n}^{M} \rightarrow J$ with continuity modulus $\varpi$. Again, this guarantees that, if $M_{\mathcal{U}}$ is the ultraproduct $\prod_{\mathcal{U}} M_{i}$, the function $D_{1}^{M_{\mathcal{U}}} \times \cdots \times D_{n}^{M_{\mathcal{U}}} \rightarrow J$

$$
\left.\left(\left[\boldsymbol{a}^{(1)}\right], \ldots,\left[\boldsymbol{a}^{(n)}\right]\right) \mapsto \lim _{i \rightarrow \mathcal{U}} \varphi^{M_{i}}\left(a_{i}^{(1)}, \ldots, a_{i}^{(n)}\right)\right]
$$

is well defined and uniformly continuous with modulus $\varpi$. Furthermore, an induction on the complexity of $\varphi$ shows that such a function coincides with the interpretation of $\varphi$ in $M_{\mathcal{U}}$. Summarizing, we have the following statement, which is the content of Łos' theorem.

Theorem 4.1. Let $L$ be a language, and $\mathcal{U}$ be an ultrafilter on a set $I$. Fix an $I$-sequence $\left(M_{i}\right)_{i \in I}$ of $L$-structures, and denote by $M_{\mathcal{U}}$ their ultraproduct $\prod_{\mathcal{U}} M_{i}$. Then for any L-formula $\varphi\left(x_{1}, \ldots, x_{n}\right)$ with free variables within $x_{1}, \ldots, x_{n}$ with domains $D_{1}, \ldots, D_{n}$, and for every $\left(\left[\boldsymbol{a}^{(1)}\right], \ldots,\left[\boldsymbol{a}^{(n)}\right]\right) \in D_{1}^{M_{\mathcal{U}}} \times \cdots \times D_{n}^{M_{\mathcal{U}}}$ one has that

$$
\varphi^{M_{\mathcal{U}}}\left(\left[\boldsymbol{a}^{(1)}\right], \ldots,\left[\boldsymbol{a}^{(n)}\right]\right)=\lim _{i \rightarrow \mathcal{U}} \varphi^{M_{i}}\left(a^{(1)}, \ldots, a_{i}^{(n)}\right) .
$$

In particular, if $\varphi$ is a sentence, then

$$
\varphi^{M_{\mathcal{U}}}=\lim _{i \rightarrow \mathcal{U}} \varphi^{M_{i}}
$$

It follows from Łos' theorem that and axiomatizable class of $\mathrm{C}^{*}$-algebras is closed under ultraproducts. From Theorem 4.1 one can deduce that the same conclusions hold for definable predicates rather than formulas. Using this fact, one can reformulate semantically the assertion that a predicate $P$ is stable in terms of ultraproducts, as follows: 
Proposition 4.2. Suppose that $P(\bar{x})$ is a definable predicate for the elementary class of $L$-structures $\mathcal{C}$. Then the following assertions are equivalent:

(1) $P(\bar{x})$ is stable;

(2) for any sequence $\left(M_{n}\right)_{n \in \mathbb{N}}$ of structures in $\mathcal{C}$, for any nonprincipal ultrafilter $\mathcal{U}$ over $\mathbb{N}$, any tuple $\bar{a}$ in $M:=\prod_{\mathcal{U}} M_{n}$ satisfying the condition $P(\bar{x})=0$ admits a representative sequence $\left(\bar{a}^{(n)}\right)_{n \in \mathbb{N}}$ of tuples $\bar{a}^{(n)}$ in $M_{n}$ such that $(\mathcal{U} n), P^{M_{n}}\left(\bar{a}^{(n)}\right)=0$.

Proof. We prove that (1) implies (2). Suppose that $P(\bar{x})$ is stable. Thus for every $m \in \mathbb{N}$ there exists $\delta_{m} \in\left(0,2^{-m}\right]$ such that, if $M$ is a structure in $\mathcal{C}$ and $\bar{b}$ is a tuple in $M$ satisfying the condition $|P(\bar{x})| \leq \delta_{m}$, then there is a tuple $\bar{a}$ in $M$ such that $d(\bar{a}, \bar{b}) \leq 2^{-m}$, and $\bar{a}$ satisfies the condition $P(\bar{x})=0$.

Suppose that $\mathcal{U},\left(M_{n}\right)_{n \in \mathbb{N}}$, and $\overline{\boldsymbol{a}}$ are as in (2). Fix any representative sequence $\left(\bar{b}^{(n)}\right)_{n \in \mathbb{N}}$ of $\overline{\boldsymbol{a}}$. Then, for every $m \in \mathbb{N},(\mathcal{U} n), \bar{b}^{(n)}$ satisfies the condition $|P(\bar{x})| \leq \delta_{m}$. Thus, for every $m \in \mathbb{N}$, the set

$$
J_{m}:=\left\{n \in \mathbb{N}: n \geq m \text { and } \bar{b}^{(n)} \text { satisfies the condition }|P(\bar{x})| \leq \delta_{m}\right\}
$$

belongs to $\mathcal{U}$. Since $\bigcap_{m \in \mathbb{N}} J_{m}=\varnothing$, for every $n \in \mathbb{N}$ there exists a largest $m(n) \in \mathbb{N}$ such that $i \in J_{m(n)}$, if it exists. Otherwise, we set $m(n)=0$.

For $n \in \mathbb{N}$ such that $m(n)>0$ define $\bar{a}^{(n)}$ to be a tuple in $M_{n}$ satisfying the conditions $d\left(x, \bar{b}^{(n)}\right) \leq 2^{-m}$ and $P(\bar{x})=0$. Observe that such a tuple exists by the choice of $\delta_{m}$ and the definition of $m(n)$. If $m(n)=0$ define $\bar{a}^{(n)}$ arbitrarily. If $m \in \mathbb{N}$, then we have that the set of $n \in \mathbb{N}$ such that $d\left(a^{(n)}, \bar{b}^{(n)}\right) \leq 2^{-m}$ and $P^{M_{n}}\left(\bar{a}^{(n)}\right)=0$ contains $J_{m}$, which belongs to $\mathcal{U}$. Therefore $\left(\bar{a}^{(n)}\right)$ is a representative sequence for $\overline{\boldsymbol{a}}$. This concludes the proof that (1) implies (2). The converse implication can be easily proved reasoning by contradiction.

4.3. Ultraproducts of $\mathbf{C}^{*}$-algebras. The general notion of ultraproduct in the logic for metric structures recovers the usual notion of ultraproduct of $\mathrm{C}^{*}$-algebras, when these are considered as structures in the language $L^{\mathrm{C}^{*}}$ introduced in Subsection 2.4. Explicitly, suppose that $\mathcal{U}$ is an ultrafilter over a set $I$, and $\left(A_{i}\right)_{i \in I}$ is an $I$-sequence of $\mathrm{C}^{*}$-algebras. Then one can let $\ell^{\infty}\left(A_{i}\right)_{i \in I}$ be the $\mathrm{C}^{*}$-algebra consisting of all bounded sequences $\boldsymbol{a} \in \prod_{i \in I} A_{i}$ endowed with the supremum norm $\|\boldsymbol{a}\|=\sup _{i \in I}\left\|a_{i}\right\|$. This $\mathrm{C}^{*}$-algebra contains the closed twosided ideal $J_{\mathcal{U}}$ consisting of those elements $\boldsymbol{a} \in \ell^{\infty}\left(A_{i}\right)_{i \in I}$ such that $\lim _{i \rightarrow \mathcal{U}}\left\|a_{i}\right\|=0$. Then $\prod_{\mathcal{U}} A_{i}$ is by definition the quotient of $\ell^{\infty}\left(A_{i}\right)_{i \in I}$ by $J_{\mathcal{U}}$. If $[\boldsymbol{a}]$ is the image in $\prod_{\mathcal{U}} A_{i}$ of an element $\boldsymbol{a}$ of $\ell^{\infty}\left(A_{i}\right)_{i \in I}$, then $\|[\boldsymbol{a}]\|=\lim _{i \rightarrow \mathcal{U}}\left\|a_{i}\right\|$. This clearly shows that such a notion of ultraproduct indeed coincides with the notion of ultraproduct of $\mathrm{C}^{*}$-algebras as $L^{\mathrm{C}^{*}}$-structures.

Let now $A$ be a $\mathrm{C}^{*}$-algebra, and $\mathcal{U}$ is an ultrafilter. One can then consider the ultrapower $A^{\mathcal{U}}$, and identify $A$ as a $\mathrm{C}^{*}$-subalgebra of $A^{\mathcal{U}}$ via the diagonal embedding. The relative commutant $A^{\prime} \cap A^{\mathcal{U}}$ is the set of elements $a$ of $A^{\mathcal{U}}$ that commute with every element of $A$.

4.4. Quantifier-free formulas and weakly semiprojective $\mathbf{C}^{*}$-algebras . Suppose that $L$ is a language. An $L$-formula $\varphi$ is quantifier-free if no quantifier appears in $\varphi$. Equivalently, $\varphi$ is of the form $q\left(\varphi_{1}, \ldots, \varphi_{n}\right)$ where $q: \mathbb{R}^{n} \rightarrow \mathbb{R}$ is a continuous function and $\varphi_{1}, \ldots, \varphi_{n}$ are atomic formulas. The notion of positive quantifier-free formula is defined similarly, where one furthermore demands that the connective $q: \mathbb{R}^{n} \rightarrow \mathbb{R}$ be nondecreasing, in the sense that $q(\bar{s}) \leq q(\bar{r})$ whenever $\bar{s}, \bar{r} \in \mathbb{R}^{n}$ satisfy $s_{i} \leq r_{i}$ for $i=1,2, \ldots, n$.

One can then define the notion of (positive) quantifier-free definable predicate, by replacing arbitrary formulas with (positive) quantifier-free ones. A condition $\varphi(\bar{x}) \leq r$ is then called quantifier-free if the definable predicate $\varphi(\bar{x})$ is quantifier-free. A (positive) quantifier-free definable set is then a definable set, whose definability is witnessed by a (positive) quantifier-free definable predicate. For instance, the unitary group or the set of projections in a $\mathrm{C}^{*}$-algebra are positive quantifier-free definable sets.

Let us consider now the language of $\mathrm{C}^{*}$-algebras $L^{\mathrm{C}^{*}}$, and let $\varphi(\bar{x})$ be a positive quantifier-free definable predicate. One can then define the universal $C^{*}$-algebra $A_{\varphi}$, if it exists, satisfying the condition $\varphi(\bar{x})=0$. This is a $\mathrm{C}^{*}$-algebra $A_{\varphi}$ containing a tuple $\bar{a}$ satisfying the condition $\varphi(\bar{x})=0$ and generating $A_{\varphi}$ as a $\mathrm{C}^{*}$-algebra, which satisfies the following universal property: if $B$ is any $\mathrm{C}^{*}$-algebra, and $\bar{b}$ is a tuple in $B$ satisfying $\varphi(\bar{x})=0$, then there exists a unital *-homomorphism $\Phi: A_{\varphi} \rightarrow B$ such that $\Phi(\bar{a})=\bar{b}$. For instance, in the case of the condition $\max \left\{\left\|x^{*} x-1\right\|,\left\|x x^{*}-1\right\|\right\}=0$ whose zeroset is the unitary group, the universal $\mathrm{C}^{*}$-algebra is the algebra $C(\mathbb{T})$ of continuous functions over the set $\mathbb{T}$ of complex numbers of modulus 1 . For the condition

$$
\max \left\{\left\|x_{i}^{2}-x_{i}\right\|,\left\|x_{i}-x_{i}^{*}\right\|,\left\|x_{i} x_{j}\right\|: 1 \leq i \neq j \leq n\right\}=0
$$


whose zeroset is the set of $n$-tuples of pairwise orthogonal projections, the universal $\mathrm{C}^{*}$-algebra is $\mathbb{C}^{n}$.

One can equivalently reformulate stability of a positive quantifier-free definable predicate $\varphi(\bar{x})$ in terms of properties of the universal $\mathrm{C}^{*}$-algebra $A_{\varphi}$, if it exists. Suppose that $\left(B_{n}\right)$ is a sequence of $\mathrm{C}^{*}$-algebras, and $\mathcal{U}$ is an ultrafilter over $\mathbb{N}$. Given an element $J$ of $\mathcal{U}$, one can define a canonical quotient mapping $\pi_{J}: \ell^{\infty}\left(B_{n}\right)_{n \in J} \rightarrow$ $\prod_{\mathcal{U}} B_{n}$ mapping $\left(a_{n}\right)_{n \in J}$ to the element of $\prod_{\mathcal{U}} B_{n}$ having $\left(a_{n}\right)_{n \in J}$ as representing sequence.

Definition 4.3. A $C^{*}$-algebra $A$ is weakly semiprojective if, for every sequence $\left(B_{n}\right)_{n \in \mathbb{N}}$ of $\mathrm{C}^{*}$-algebras, for every ultrafilter $\mathcal{U}$ over $\mathbb{N}$, and for every unital ${ }^{*}$-homomorphism $\Phi: A \rightarrow \prod_{\mathcal{U}} B_{n}$, there exists $J \in \mathcal{U}$ and a unital *-homomorphism $\hat{\Phi}: A \rightarrow \ell^{\infty}\left(B_{n}\right)_{n \in J}$ such that $\Phi=\pi_{J} \circ \hat{\Phi}$.

Suppose now that $\varphi$ is a positive quantifier-free definable predicate such that the condition $\varphi(\bar{x})=0$ has a universal C*-algebra $A_{\varphi}$. Then one can easily deduce from Proposition 4.2 the following characterization, which recovers [61, Theorem 4.1.4].

Theorem 4.4. The positive quantifier-free definable predicate $\varphi$ is stable if and only if $A_{\varphi}$ is weakly semiprojective.

As an application of Theorem 4.4, one can consider the $\mathrm{C}^{*}$-algebra $M_{n}(\mathbb{C})$ of complex $n \times n$ matrices endowed with the operator norm. This is the universal $\mathrm{C}^{*}$-algebra associated with the definable predicate $\varphi_{M_{n}(\mathbb{C})}\left(x_{i j}\right)$ given by

$$
\max \left\{\left\|x_{i j} x_{k \ell}-\delta_{j k} x_{i \ell}\right\|,\left|1-\left\|x_{i j}\right\|\right|,\left\|x_{i j}^{*}-x_{j i}\right\|,\left|1-\sum_{m=1}^{n} x_{m, m}\right|: 1 \leq i, j, k, \ell \leq n\right\} .
$$

The zeroset of $\varphi_{M_{n}(\mathbb{C})}$ in a $\mathrm{C}^{*}$-algebra $A$ is the set of matrix units for a unital copy of $M_{n}(\mathbb{C})$ inside $A$. The $\mathrm{C}^{*}$ algebra $M_{n}(\mathbb{C})$ is weakly semiprojective [61, Theorem 10.2.3], hence the predicate $P$ is stable. More generally, given a finite-dimensional $\mathrm{C}^{*}$-algebra $F$, i.e. a finite sum $M_{n_{1}}(\mathbb{C}) \oplus \cdots \oplus M_{n_{\ell}}(\mathbb{C})$, one can consider the definable predicate $P_{F}$ whose zeroset in a $\mathrm{C}^{*}$-algebra $A$ is the set of matrix units for a unital copy of $F$ inside $A$. The fact that $F$ is weakly semiprojective shows that such a definable predicate $P_{F}$ is stable.

4.5. Saturation. One important feature of ultrapowers is their being very "rich". To make this precise, we introduce the notion of countably saturated structure. Suppose that $L$ is a language, and $M$ is an $L$-structure. Let $A$ be a subset of $M$. Then one can consider the language $L(A)$ obtained starting from $L$ by adding a constant symbol $c_{a}$ for every element $a$ of $A$. Then one can canonically regard $M$ as an $L(A)$ structure by defining the interpretation of the constant symbol $c_{a}$ in $M$ to be $a$ itself. We refer to formulas in the language $L(A)$ as $L$-formulas with parameters from $A$.

If $\mathcal{C}$ is a class of $L$-structure, then we say that $L$ is separable for $\mathcal{C}$ if, for every $n \in \mathbb{N}$ and variables $x_{1}, \ldots x_{n}$ of domains $D_{1}, \ldots, D_{n}$, the seminorm on the space of $L$-formulas $\varphi\left(x_{1}, \ldots, x_{n}\right)$ defined by

$$
\|\varphi\|=\sup \left\{|\varphi(\bar{a})|: M \in \mathcal{C}, \bar{a} \in D_{1}^{M} \times \cdots \times D_{n}^{M}\right\}
$$

is separable.

Recall that and $L$-condition in the variables $x_{1}, \ldots, x_{n}$ of domains $D_{1}, \ldots, D_{n}$ is an expression of the form $\varphi\left(x_{1}, \ldots, x_{n}\right) \leq r$ where $\varphi$ is an $L$-formula - or, more generally, a definable predicate - in the free variables $x_{1}, \ldots, x_{n}$, and $r \in \mathbb{R}$. If $M$ is an $L$-structure and $\bar{a} \in D_{1}^{M} \times \cdots \times D_{n}^{M}$, then $\left(a_{1}, \ldots, a_{n}\right)$ realizes such a condition if $\varphi^{M}\left(a_{1}, \ldots, a_{n}\right) \leq r$. A collection of $L$-conditions in the variables $\bar{x}=\left(x_{n}\right)_{n \in \mathbb{N}}$ is called an $L$-type

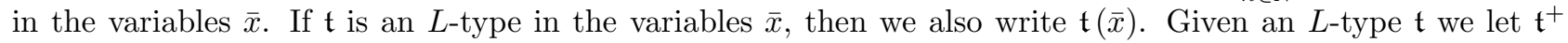
be the $L$-type consisting of conditions $\varphi(\bar{x}) \leq r+\varepsilon$ where $\varphi(\bar{x}) \leq r$ is a condition in $\mathfrak{t}$ and $\varepsilon>0$. Given an $L$-structure $M$ and a tuple $\bar{a}$ in $M$, one defines the complete $L$-type of $\bar{a}$ to be the type $\mathfrak{t}(\bar{x})$ consisting of all the $L$-conditions that are satisfied by $\bar{a}$.

Definition 4.5. Suppose that $\mathfrak{t}$ is an $L$-type in the variables $\bar{x}$ and $M$ is an $L$-structure. Then $\mathfrak{t}$ is:

- realized in $M$ if there exist $a_{n} \in D_{n}^{M}$ for $n \in \mathbb{N}$ such that the sequence $\left(a_{n}\right)$ realizes every condition in $\mathfrak{t}$;

- approximately realized in $M$ if every finite set of conditions in $\mathfrak{t}^{+}$is realized in $M$.

The notion realized and approximately realized types allows one to define the property of countable saturation for structures, which formalizes the intuitive idea of being "rich".

Definition 4.6. An $L$-structure $M$ is countably saturated if for every separable subset $A$ of $M$ and for every $L(A)$-type $\mathfrak{t}$, if $\mathfrak{t}$ is approximately realized in $M$ then $\mathfrak{t}$ is realized in $M$. 
A fundamental feature of ultraproducts associated with countably incomplete ultrafilters is their being countably saturated.

Proposition 4.7. Let $L$ be a first-order language, and $\mathcal{C}$ is a class of $L$-structures such that $L$ is separable for $\mathcal{C}$. If $\mathcal{U}$ is a countably incomplete filter on a set $I$, and $\left(M_{i}\right)_{i \in I}$ is an I-sequence of structures in $\mathcal{C}$, then the ultraproduct $\prod_{\mathcal{U}} M_{i}$ is countably saturated.

Proof. Set $M:=\prod_{\mathcal{U}} M_{i}$. Suppose that $A$ is a separable subset of $M$. We need to show that if an $L(A)$-type $\mathfrak{t}$ is approximately realized in $M$, then it is realized in $M$. For every $[\boldsymbol{a}] \in A$ fix a representative sequence $\boldsymbol{a}=\left(a_{i}\right)_{i \in I}$. For every $i \in I$ we can regard $M_{i}$ as an $L(A)$-structure by declaring the interpretation $c_{\boldsymbol{a}}$ of $\boldsymbol{a}$ to be equal to $a_{i}$. Observe that, since $A$ is separable and $L$ is separable for $\mathcal{C}$, we have that $L(A)$ is separable for $\left\{M_{i}: i \in I\right\} \cup\{M\}$. Thus after replacing $L$ with $L(A)$ we can assume without loss of generality that $A$ is empty.

Let thus $\mathfrak{t}$ be an $L$-type in the variables $\bar{x}$ of domains $\bar{D}$ which is approximately realized in $M$. Since $L$ is separable for $\mathcal{C}$, we can assume without loss of generality that $\mathfrak{t}$ consists of a sequence of conditions $\varphi_{n}\left(x_{1}, \ldots, x_{n}\right) \leq 0$ for $n \in \mathbb{N}$. Since by assumption $\mathcal{U}$ is countably incomplete, we can fix a decreasing sequence $\left(I_{n}\right)_{n \in \mathbb{N}}$ of elements of $\mathcal{U}$ with empty intersection.

For every $n \in \mathbb{N}$ define $\psi_{n}$ to be the sentence $\inf _{x_{1}, \ldots, x_{n}} \max \left\{\varphi_{1}\left(x_{1}\right), \ldots, \varphi_{n}\left(x_{1}, \ldots, x_{n}\right)\right\}$. By assumption, $\mathfrak{t}$ is approximately realized in $M$, hence $\psi_{n}^{M}<\frac{1}{n}$. Therefore by Łos' theorem, the set

$$
J_{n}=\left\{i \in I_{n}: \psi_{n}^{M}<\frac{1}{n}\right\}
$$

belongs to $\mathcal{U}$. Observe that $\left(J_{n}\right)_{n \in \mathbb{N}}$ is a decreasing sequence of elements of $\mathcal{U}$ with empty intersection. Thus every $i \in I$ only belongs to finitely many of the $J_{n}$ 's, and hence there exists a largest element $n(i)$ of $\mathbb{N}$ such that $i \in J_{n(i)}$. If $i$ does not belong to any of the $J_{n}$ 's then we set $n(i)=0$.

Define now, for $i \in I$, if $n(i)>0, \bar{a}_{i}=\left(a_{1, i}, \ldots, a_{n(i), i}\right) \in D_{1}^{M_{i}} \times \cdots \times D_{n(i)}^{M_{i}}$ such that $\psi_{n(i)}\left(\bar{a}_{i}\right)<1 / n(i)$. For $i \in I$ such that $n(i)=0$ define $\bar{a}_{i} \in \bar{D}^{M_{i}}$ arbitrarily. Now, it is clear from the definition of $n(i)$ that, for every $n \in \mathbb{N}$ and $i \in J_{n}, n(i) \geq n$. Let then $\boldsymbol{a}_{n}$ for $n \in \mathbb{N}$ be the element of $\prod_{\mathcal{U}} M_{i}$ with representative sequence $\left(a_{n, i}\right)_{i \in I}$. Since $J_{n} \in \mathcal{U}$, we have that $(\mathcal{U} i), \psi_{n}^{M_{i}}\left(\bar{a}_{i}\right) \leq 1 / n$. Therefore by Łos' theorem, $\psi_{n}^{M}(\overline{\boldsymbol{a}}) \leq 1 / n$ for every $n \in \mathbb{N}$. Since this holds for every $n \in \mathbb{N}$, we have that $\varphi_{n}^{M}(\overline{\boldsymbol{a}}) \leq 0$ for every $n \in \mathbb{N}$. This shows that $\overline{\boldsymbol{a}}$ witnesses that the type $\mathfrak{t}$ is realized in $M$.

A type $\mathfrak{t}$ is called (positive) quantifier-free if all the conditions in $\mathfrak{t}$ involve (positive) quantifier-free formulas. One can then naturally define the notion of (positive) quantifier-free countably saturated structure as in Definition 4.6, by replacing arbitrary types with (positive) quantifier-free ones.

It is easy to see that, if $M$ is a quantifier-free countably saturated structure, and $\mathfrak{t}$ is a quantifier-free type, then the set of realizations of $\mathfrak{t}$ in $M$ is quantifier-free countably saturated. Particularly, if $A$ is a $\mathrm{C}^{*}$-algebra, and $\mathcal{U}$ is a countably incomplete ultrafilter, then the relative commutant $A^{\prime} \cap A^{\mathcal{U}}$ is quantifier-free countably saturated.

4.6. Elementary equivalence and elementary embeddings. Elementary equivalence is a key notion in model theory. Roughly speaking, it asserts that two structures are indistinguishable as long as first-order properties (i.e. properties that are captured by formulas) are concerned. To make this precise, let $L$ be a language, and $M$ be an $L$-structure. The theory of $M$ is the multiplicative functional $\varphi \mapsto \varphi^{M}$ defined on the space of $L$-sentences, which maps an $L$-sentence to its interpretation in $M$.

Definition 4.8. Two $L$-structures are elementarily equivalent if they have the same theory.

A straightforward induction on formulas shows that two isomorphic structures are, in particular, elementarily equivalent. It follows from Łos' theorem that if $M$ is an $L$-structure and $\mathcal{U}$ is an ultrafilter, then $M$ is elementarily equivalent to $M^{\mathcal{U}}$. In particular, if two structures have isomorphic ultrapowers, then they are elementarily equivalent. The continuous version of a classical result of Keisler and Shelah asserts that, in fact, the converse holds as well. The notion of elementary embedding is tightly connected with elementary equivalence.

Definition 4.9. An embedding of $M$ to $N$ is a function $\Phi: M \rightarrow N$ such that, for every domain $D$ in $L$, the image of $D^{M}$ under $\Phi$ is contained in $D^{N}$, and such that $\varphi(\Phi(\bar{a}))=\varphi(\bar{a})$ for every atomic formula $\varphi(\bar{x})$ in the variables $\bar{x}$ of domains $\bar{D}$ and $\bar{a} \in \bar{D}^{M}$. 
We say that $M$ is a substructure of $N$ if $M \subset N$ and the inclusion map is an embedding. Clearly, after renaming the elements of $M$, one can always assume that a given embedding $\Phi: M \rightarrow N$ is simply the inclusion map.

Suppose that $M \subset N$ is a substructure. Then one can regard $N$ as an $L(M)$-structure in the obvious way, by interpreting the constant symbol $c_{a}$ associated with $a \in M$ as $a$ itself, regarded as an element of $N$.

Definition 4.10. Suppose that $M \subset N$ is a substructure. Then $M$ is an elementary substructure of $N$ if $M$ and $N$ are elementarily equivalent as $L(M)$-structures.

The notion of elementary embedding $\Phi: M \rightarrow N$ is defined analogously. In this case, one can consider $N$ as an $L(M)$-structure by interpreting $c_{a}$ for $a \in M$ as $\Phi(a)$.

If $M$ is any $L$-structure and $\mathcal{U}$ is an ultrafilter, then there is a canonical embedding $\Delta_{M}: M \rightarrow M^{\mathcal{U}}$ obtained by mapping an element $a$ of $M$ to the element of $M^{\mathcal{U}}$ that admits the sequence constantly equal to $a$ as representing sequence. It is a consequence of Łos' theorem that this is in fact an elementary embedding. A useful criterion to verify that an inclusion $M \subset N$ is elementary is the following Tarski-Vaught test.

Proposition 4.11. Suppose that $M, N$ are L-structures such that $M \subset N$. Assume that for every L-formula $\varphi(\bar{x}, y)$, where $\bar{x}$ are variables with domains $\bar{D}$ and $y$ has domain $D$, and for every tuple $\bar{a} \in \bar{D}^{M}$ one has that

$$
\inf \left\{\varphi(\bar{a}, b): b \in D^{N}\right\}=\inf \left\{\varphi(\bar{a}, b): b \in D^{M}\right\} .
$$

Then $M$ is an elementary substructure of $N$.

Proof. One needs to show by induction on the complexity of a given formula $\psi(\bar{x})$ and tuple $\bar{a}$ in $M$, where $\bar{x}$ are variables with domains $\bar{D}$ and $\bar{a} \in \bar{D}^{M}$, that $\psi^{M}(\bar{a})=\psi^{N}(\bar{a})$. The base case when $\psi$ is atomic (or quantifier-free) is obvious. The assumption is used to deal with the quantifier case.

4.7. Existential equivalence and existential embeddings. In many cases, it is sufficient, and useful, to consider a suitably restricted class of formulas. We have already considered the class of quantifier-free formulas. The next natural restricted class of formulas consists of existential formulas. These are the formulas of the form $\inf _{\bar{x}} \varphi$ where $\varphi$ is a quantifier-free formula. The name "existential" is due to the fact that inf is regarded as the continuous analog of the existential quantifier $\exists$ from the logic for discrete structures.

An even more restrictive class consists of the positive existential formulas, which are those existential formulas of the form $\inf _{\bar{x}} q\left(\varphi_{1}, \ldots, \varphi_{n}\right)$ where $\varphi_{1}, \ldots, \varphi_{n}$ are atomic formulas, and $q: \mathbb{R}^{n} \rightarrow \mathbb{R}$ is a continuous function which is nondecreasing, in the sense that $q(\bar{r}) \leq q(\bar{s})$ if $\bar{r}, \bar{s} \in \mathbb{R}^{n}$ are such that $r_{i} \leq s_{i}$ for $i \in\{1,2, \ldots, n\}$. Universal formulas and positive universal formulas are defined and characterized in a similar fashion, by replacing inf with sup.

One can then define the (positive) existential theory of a structure $M$ to be the functional $\varphi \mapsto \varphi^{M}$ defined on the space of (positive) existential formulas. It follows from Los' theorem and countable saturation of ultrapowers that, if $M, N$ are separable structures and $\mathcal{U}$ is a countably incomplete ultrafilter, then $M$ and $N$ have the same existential theory if and only if $M$ embeds into $N^{\mathcal{U}}$ and $N$ embeds into $M^{\mathcal{U}}$. One can similarly characterize the property of having the same positive existential theory in terms of ultraproducts and morphisms.

Definition 4.12. Suppose that $M, N$ are structures and $\Phi: M \rightarrow N$ is a function. Then $\Phi$ is a morphism if for every domain $D$, the image of $D^{M}$ under $\Phi$ is contained in $D^{N}$, and such that $\varphi(\Phi(\bar{a})) \leq \varphi(\bar{a})$ for every atomic formula $\varphi(\bar{x})$ in the variables $\bar{x}$ of domains $\bar{D}$ and $\bar{a} \in \bar{D}^{M}$.

One can show using countable saturation of ultraproducts and Łos' theorem that, if $M$ and $N$ are separable structures and $\mathcal{U}$ is a countably incomplete ultrafilter, then $M$ and $N$ have the same positive existential theory if and only if $M$ admits a morphism to $N^{\mathcal{U}}$ and $N$ admits a morphism to $M^{\mathcal{U}}$. More generally, one has that $\varphi^{M} \geq \varphi^{N}$ for every existential (respectively, positive existential) sentence if and only if there is an embedding (respectively, a morphism) from $M$ to $N^{\mathcal{U}}$.

The notions of (positively) existential substructure and (positively) existential embedding are defined in the same fashion as elementary substructure and elementary embeddings, replacing arbitrary formulas with (positive) existential formulas. The following characterization of (positively) existential embedding follows again from Łos' theorem and countable saturation of ultraproducts.

Proposition 4.13. Suppose that $M, N$ are structures, and $\Phi: M \rightarrow N$ is an embedding. Fix a countably incomplete ultrafilter $\mathcal{U}$. The following assertions are equivalent:

(1) $\Phi$ is an existential (respectively, positively existential) embedding; 
(2) there exists an embedding (respectively, a morphism) $\Psi: N \rightarrow M^{\mathcal{U}}$ such that $\Psi \circ \Phi$ is equal to the diagonal embedding $\Delta_{M}: M \rightarrow M^{\mathcal{U}}$.

Suppose now that $\mathcal{C}$ is an elementary class of $L$-structures. An $L$-structure $M$ is said to be (positively) existentially closed in $\mathcal{C}$ if it belongs to $\mathcal{C}$ and, whenever $N$ is a structure in $\mathcal{C}$ containing $M$ as a substructure, the inclusion $M \subset N$ is existential.

4.8. Positively existential embeddings of $\mathbf{C}^{*}$-algebras. In the case of $\mathrm{C}^{*}$-algebras regarded as $L^{\mathrm{C}^{*}}$ structures, an embedding is an injective unital *-homomorphism, and a morphism is a unital *-homomorphism. The general notion of (positively) existential embedding yields a notion of (positively) existential embedding between $\mathrm{C}^{*}$-algebras. If $A, B$ are separable $\mathrm{C}^{*}$-algebras, an embedding $\Phi: A \rightarrow B$ is positively existential if and only if it is sequentially split in the sense of [9].

Using positively existential embeddings, one can give a model-theoretic description of relative commutants, as follows.

Proposition 4.14. Suppose that $A, C$ are separable $C^{*}$-algebras, and $\mathcal{U}$ is a countably incomplete ultrafilter. Then the following assertions are equivalent:

(1) the embedding $1_{C} \otimes \operatorname{id}_{A}: A \rightarrow C \otimes_{\max } A$ is positively existential;

(2) there exists a morphism from $C$ to $A^{\prime} \cap A^{\mathcal{U}}$.

Proof. $(1) \Rightarrow(2)$ We identify $A$ with its image $1 \otimes_{\max } A$ inside $C \otimes_{\max } A$. By countable saturation of $A^{\mathcal{U}}$ it suffices to prove the following. Suppose that $\varphi(\bar{x})$ is a positive existential $L^{\mathrm{C}^{*}}$-formula in the variables $x_{1}, \ldots, x_{n}, \varepsilon>0$, $a_{1}, \ldots, a_{k}$ is a tuple in $A$, and $\bar{c}$ is a tuple in $C$ satisfying the condition $\varphi(\bar{x}) \leq 0$. Then there exists a tuple in $A$ satisfying the $L^{\mathrm{C}^{*}}(A)$-condition $\psi(\bar{x}) \leq \varepsilon$ where $\psi(\bar{x})$ is the $L^{\mathrm{C}^{*}}(A)$-formula

$$
\max \left\{\varphi(\bar{x}),\left\|x_{i} a_{j}-a_{j} x_{i}\right\|: i=1,2, \ldots, n \text { and } j=1,2, \ldots, k\right\} .
$$

Considering the tuple $c_{i} \otimes 1_{A} \in C \otimes A$ for $i=1,2, \ldots, n$ shows that the $L^{\mathrm{C}^{*}}(A)$-condition $\psi(\bar{x}) \leq 0$ is satisfied in $C \otimes A$. Since the inclusion $A \subset C \otimes \max A$ is positively existential by assumption, we conclude that the $L^{\mathrm{C}^{*}}(A)$-condition $\psi(\bar{x}) \leq \varepsilon$ is satisfied in $A$. This concludes the proof.

$(2) \Rightarrow(1)$ Suppose that there exists a morphism $\eta: C \rightarrow A^{\prime} \cap A^{\mathcal{U}}$. Then the function $\left(A^{\prime} \cap A^{\mathcal{U}}\right) \times A \rightarrow A^{\mathcal{U}}$ given by $\left(\left[a_{i}\right], b\right) \mapsto\left[a_{i} b\right]$ induces by the universal property of maximal tensor products a morphism $\Psi$ : $\left(A^{\prime} \cap A^{\mathcal{U}}\right) \otimes_{\max } A \rightarrow A^{\mathcal{U}}$. One can then define $\hat{\Psi}:=\Psi \circ\left(\eta \otimes \mathrm{id}_{A}\right): C \otimes_{\max } A \rightarrow A^{\mathcal{U}}$. Observe that this is a morphism such that $\hat{\Psi} \circ\left(1_{C} \otimes \mathrm{id}_{A}\right)$ is the diagonal embedding of $A$ into $A^{\mathcal{U}}$. This shows that $1_{C} \otimes \mathrm{id}_{A}$ is a positively existential embedding.

\section{The efFect of the Continuum Hypothesis}

5.1. The Continuum Hypothesis. The continuum $\mathfrak{c}$ is, by definition, the cardinality of the set $\mathbb{R}$ of real numbers. The Continuum Hypothesis $(\mathrm{CH})$ is the assertion that $\mathfrak{c}$ coincides with the least uncountable cardinal $\aleph_{1}$. A famous open problem in set theory asked whether the Continuum Hypothesis holds, or more precisely whether it follows from the usual axioms for set theory known as Zermelo-Frankel axioms with Choice (ZFC). In 1940 Gödel [51] showed that the Continuum Hypothesis is consistent with ZFC, in the sense that it can be added to ZFC without leading to a contradiction (assuming that ZFC itself is not contradictory). In the early 1960s, Cohen developed the method of forcing, and used it to show that the negation of the Continuum Hypothesis (the assertion that $\mathfrak{c}$ is strictly larger than $\aleph_{1}$ ) is also consistent with ZFC [21, 22]. These results together imply that the Continuum Hypothesis is independent of ZFC, in the sense that it can not be either proved nor disproved from the axioms of ZFC.

The value of the continuum turns out to be irrevelant for what concerns sufficiently simple statement. As a rule of thumb, any "reasonable statement" concerning separable $\mathrm{C}^{*}$-algebras which can be proved assuming the Continuum Hypothesis, can also be proved without the Continuum Hypothesis. (This assertion can be made precise in set theory through the notion of absoluteness, and it is the subject of several absoluteness results such as Shoenfield's absoluteness theorem [73].)

On the other hand, the value of the continuum, or more generally additional set-theoretic axioms, can have a deep influence on the structure and properties of "massive $\mathrm{C}^{*}$-algebras". Paradigmatic in this sense is the question of whether all automorphisms of the Calkin algebra $\mathcal{Q}$ are inner. Recall that $\mathcal{Q}$ is the quotient of the algebra $B(H)$ of bounded linear operators on the separable Hilbert space $H$ by the closed two-sided ideal of compact operators. Even when $H$ is separable, $\mathcal{Q}$ is nonseparable, and in fact it has density character $\mathfrak{c}$. 
Originally posed by Brown-Douglas-Fillmore in [16], this problem was initially addressed in 2007 by PhillipsWeaver [67], who showed that, assuming $\mathrm{CH}, \mathcal{Q}$ has an automorphism which is not inner. Later on, Farah has proved that, under different set-theoretic assumptions, which imply in particular the negation of $\mathrm{CH}$, all the automorphisms of the Calkin algebra are inner [32,33]. These results have later been generalized in $[23,43,46,83]$ to other massive $\mathrm{C}^{*}$-algebras, which are obtained as corona algebras of separable $\mathrm{C}^{*}$-algebras.

Another problem which is sensitive of the value of the continuum concerns the number of ultrapowers of a fixed separable $\mathrm{C}^{*}$-algebra infinite-dimensional $\mathrm{C}^{*}$-algebra $A$ with respect to nonprincipal ultrafilters over $\mathbb{N}$. CH implies that all such ultrapowers of $A$ are isomorphic. On the other hand, as shown by Farah-HartSherman [38], if $\mathrm{CH}$ fails then there exist two nonisomorphic such ultrapowers of $A$ (in fact, there exist $2^{\mathfrak{c}}$ pairwise nonisomorphic such ultrapowers of $A$, as proved by Farah-Shelah [45]). The same conclusions hold if one considers, instead of the ultrapower, the relative commutant of $A$ inside the ultrapower. The analogous question in the case of $\mathrm{II}_{1}$ factors had been posed by McDuff [62], and it has also been settled in [38].

5.2. Isomorphism of countably saturated structures. Let $L$ be a language. Recall that an $L$-structure $M$ is countably saturated if for every separable subset $A$ of $M$ and every $L(A)$-type $\mathfrak{t}(\bar{x})$, if $\mathfrak{t}(\bar{x})$ is approximately realized in $M$, then it is realized in $M$. A fundamental fact in model theory is that the any two elementarily equivalent countably saturated structures of density character $\aleph_{1}$ are isomorphic. More generally, we have the following result.

Theorem 5.1. Suppose that $L$ is a language, and let $\mathcal{C}$ be a class of $L$-structures such that $L$ is separable for $\mathcal{C}$. Consider two elementarily equivalent countably saturated structures $M, N$ in $\mathcal{C}$. Then $M$ and $N$ are isomorphic. Furthermore, if $M_{0} \subset M$ is a separable substructure, and $\Phi_{0}: M_{0} \rightarrow N$ is an elementary embedding, then $\Phi$ extends to an isomorphism $M \rightarrow N$.

Proof. We prove the second assertion, the proof of the first assertion being similar.

Since $M, N$ have density character $\aleph_{1}$, one can enumerate dense subsets $\left\{a_{\lambda}: \lambda<\omega_{1}\right\}$ of $M$ and $\left\{b_{\lambda}: \lambda<\omega_{1}\right\}$ of $N$. Define $N_{0}$ to be the range of $\Phi$. Let also $M_{\lambda}$ for $\lambda<\omega_{1}$ be $M_{0} \cup\left\{a_{\mu}: \mu<\lambda\right\} \subset M$, and similarly $N_{\lambda}$ for $\lambda<\omega_{1}$ be $N_{0} \cup\left\{b_{\mu}: \mu<\lambda\right\} \subset N$. Say that an ordinal $\lambda<\omega_{1}$ is odd if it is of the form $\mu+n$ where $\mu$ is a limit ordinal and $n \in \omega$ is odd, and it is even otherwise.

We define by recursion on $\lambda<\omega_{1}$ elements $\hat{a}_{\lambda}$ of $M$ and $\hat{b}_{\lambda}$ of $N$ such that:

(1) $\hat{a}_{\lambda}=a_{\lambda}$ if $\lambda$ is even;

(2) $\hat{b}_{\lambda}=b_{\lambda}$ if $\lambda$ is odd;

(3) the assignment $\Phi_{\lambda}: M_{\lambda} \rightarrow N_{\lambda}$ which is the identity on $M_{0}$ and such that $\Phi\left(\hat{a}_{\mu}\right)=\hat{b}_{\mu}$ for $\mu<\lambda$ is well defined and satisfies $\varphi^{N}\left(\Phi_{\lambda}(\bar{a})\right)=\varphi^{M}(\bar{b})$ for any tuple $\bar{a}$ in $M_{\lambda}$ and $L\left(M_{\lambda}\right)$-formula $\varphi(\bar{x})$, where $N_{\lambda}$ is regarded as an $L\left(N_{\lambda}\right)$-structure by interpreting the constant $c_{a}$ associated with $a \in M_{\lambda}$ as $\Phi(a)$.

Suppose that $\lambda<\omega_{1}$ and $\hat{a}_{\mu}, \hat{b}_{\mu}$ have been defined for $\mu<\lambda$ in such a way that (1),(2),(3) above hold. (Observe that (3) holds when $\lambda=0$ by the assumption that $\Phi_{0}$ is an elementary embedding). We consider the case when $\lambda$ is even, the case of $\lambda$ being odd is analogous. We then set $\hat{a}_{\lambda}=a_{\lambda}$, and the consider the complete $L\left(M_{\lambda}\right)$-type of $\hat{a}_{\lambda}$. Recall that this is the $L\left(M_{\lambda}\right)$-type $\mathfrak{t}(x)$ consisting of all the $L\left(M_{\lambda}\right)$ conditions satisfied by $\hat{a}_{\lambda}$. By the inductive assumption (3), $\mathfrak{t}(x)$ is approximately realized in $N$. Since $N$ is countably saturated, we can conclude that $\mathfrak{t}(x)$ is realized in $N$. We then define $\hat{b}_{\lambda}$ to be any realization of $\mathfrak{t}(x)$ in $N$. It is clear by definition of $\mathfrak{t}(x)$ together with the inductive assumption that such a choice indeed satisfies (3). This concludes the recursive construction.

Observe that, by (1), $\left\{\hat{a}_{\lambda}: \lambda<\omega_{1}\right\}$ is a dense subset of $M$. Similarly, by (2), $\left\{\hat{b}_{\lambda}: \lambda<\omega_{1}\right\}$ is a dense subset of $N$. Granted the construction, one can define the map $\Phi:\left\{\hat{a}_{\lambda}: \lambda<\omega_{1}\right\} \rightarrow\left\{\hat{b}_{\lambda}: \lambda<\omega_{1}\right\}$ by $\Phi\left(\hat{a}_{\lambda}\right)=\hat{b}_{\lambda}$. By (3), this extends to an isomorphism $\Phi: M \rightarrow N$, concluding the proof.

5.3. Ultrapowers and the Continuum Hypothesis. We now specialize the discussion to $\mathrm{C}^{*}$-algebras. Let $A$ be an infinite-dimensional separable $\mathrm{C}^{*}$-algebra, and $\mathcal{U}$ is a nonprincipal ultrafilter over $\mathbb{N}$. Recall that this means that $\mathcal{U}$ does not contain any finite set, which is equivalent to the assertion that $\mathcal{U}$ is countably incomplete. We consider the ultrapower $A^{\mathcal{U}}$ and the relative commutant $A^{\prime} \cap A^{\mathcal{U}}$. A basic question is: how large $A^{\mathcal{U}}$ and $A^{\prime} \cap A^{\mathcal{U}}$ are? Considering that the continuum $\mathfrak{c}$ is also the size of the set of functions from $\mathbb{N}$ to $\mathbb{N}$, since we are assuming that $A$ is separable, and that $\mathcal{U}$ is an ultrafilter over $\mathbb{N}$, it is easy to see that $A^{\mathcal{U}}$ has density character at most $\mathfrak{c}$. (Recall that the density character of a $\mathrm{C}^{*}$-algebra is the least size of a dense set.) Clearly, the same conclusion applies to $A^{\prime} \cap A^{\mathcal{U}}$, which is a $\mathrm{C}^{*}$-subalgebra of $A^{\mathcal{U}}$. We now claim that, in fact, $A^{\prime} \cap A^{\mathcal{U}}$ and hence $A^{\mathcal{U}}$ have density character exactly $\mathfrak{c}$. In order to show this, we will use the following criterion, which we formulate in the general setting of structures in an arbitrary language $L$. 
Proposition 5.2. Suppose that $L$ is a language, $\mathcal{U}$ is a nonprincipal ultrafilter over $\mathbb{N}, \mathfrak{t}(x)$ is an $L$-type in the variable $x$ with corresponding domain $D$, and $\mathcal{C}$ is a class of $L$-structure. Assume that $L$ is separable for $\mathcal{C}$. Consider a sequence $\left(M_{n}\right)_{n \in \mathbb{N}}$ of L-structures. Assume that there exist

- $\delta>0$, and

- for every $n \in \mathbb{N}$, an $n$-tuple $\bar{a}^{(n)}=\left(a_{1}^{(n)}, \ldots, a_{n}^{(n)}\right)$ of elements of $D^{M_{n}}$,

such that

(1) for every $n \in \mathbb{N}$, and $1 \leq i, j \leq n, d^{M_{n}}\left(a_{i}, a_{j}\right) \geq \delta$, and

(2) for every finite set $\mathfrak{t}_{0}(x)$ of conditions in $\mathfrak{t}(x)^{+}$, every element of $\bar{a}^{(n)}$ satisfies $\mathfrak{t}_{0}(x)$ for all but finitely many $n \in \mathbb{N}$.

Let $M$ be the ultraproduct $\prod_{\mathcal{U}} M_{n}$, and $M^{\mathfrak{t}}$ be the set of realizations of $\mathfrak{t}(x)$ in $\prod_{\mathcal{U}} M_{n}$. Then $M^{\mathfrak{t}}$ contains a family $\left(a_{i}\right)_{i<\mathfrak{c}}$ of size continuum of elements satisfying $d^{M}\left(a_{i}, a_{j}\right) \geq \delta$ for every $i, j<\mathfrak{c}$.

In fact, a more general version of Proposition 5.2 holds, where one considers a type in more than one variable.

In order to prove Proposition 5.2, we will use the following basic lemma from combinatorial set theory. Let us say that two functions $f, g: \mathbb{N} \rightarrow \mathbb{N}$ are eventually distinct if $\{n \in \mathbb{N}: f(n)=g(n)\}$ is finite.

Lemma 5.3. There exists a family $\mathcal{F}$ of size continuum of pairwise eventually distinct functions $f: \mathbb{N} \rightarrow \mathbb{N}$ satisfying $f(n) \leq n$ for every $n \in \mathbb{N}$ and $\lim _{n \rightarrow+\infty} f(n)=+\infty$.

Proof. We will use the fact that the continuum is equal to the cardinality of the collection of infinite subsets of $\mathbb{N}$. For an infinite subset $A$ of $\mathbb{N}$, define the function $f_{A}: \mathbb{N} \rightarrow \mathbb{N}$ by

$$
f_{A}(n)=\sum_{k<\left\lfloor\log _{2} n\right\rfloor} \chi_{A}(k) 2^{k} .
$$

Observe that $\left|A \cap\left[1, \log _{2} n\right]\right| \leq f_{A}(n) \leq n$ for every $n \in \mathbb{N}$. Furthermore, if $A, B$ are distinct infinite subsets of $\mathbb{N}$, then $f_{A}$ and $f_{B}$ are eventually distinct.

We can now use Lemma 5.3 to prove Proposition 5.2.

Proof of Proposition 5.2. Let $\mathcal{F}$ be a family of size continuum consisting of pairwise eventually distinct functions $f: \mathbb{N} \rightarrow \mathbb{N}$ such that $f(n) \leq n$ for every $n \in \mathbb{N}$ and $\lim _{n \rightarrow+\infty} f(n)=+\infty$. For $n \in \mathbb{N}$, let $\bar{a}^{(n)}=\left(a_{1}^{(n)}, \ldots, a_{n}^{(n)}\right)$ be the tuple of elements of $D^{M_{n}}$ given by hypothesis. Let $M$ be the ultraproduct $\prod_{\mathcal{U}} M_{n}$. For every $f \in \mathcal{F}$ define $\boldsymbol{a}^{f}$ to be the element of $D^{M}$ with representative sequence $\left(a_{f(n)}^{(n)}\right)$. The assumption that, for every finite set $\mathfrak{t}_{0}(x)$ of conditions in $\mathfrak{t}(x)^{+}$, every element of $\bar{a}^{(n)}$ satisfies $\mathfrak{t}_{0}(x)$ for all but finitely many $n \in \mathbb{N}$, and the fact that $\lim _{n \rightarrow+\infty} f(n)=+\infty$, implies by Łos' theorem that $\boldsymbol{a}^{f}$ satisfies $\mathfrak{t}(x)$.

If $f$ and $g$ are different elements of $\mathcal{F}$, then they are eventually distinct. In particular, since $\mathcal{U}$ is nonprincipal, $(\mathcal{U} n), f(n) \neq g(n)$. Hence, $(\mathcal{U} n), d\left(a_{f(n)}^{n}, a_{g(n)}^{n}\right) \geq \delta$. By Los' theorem again, we then have $d\left(\boldsymbol{a}^{f}, \boldsymbol{a}^{g}\right) \geq \delta$. This concludes the proof.

Using Proposition 5.2 we can give a sufficient condition for a $\mathrm{C}^{*}$-algebra $A$ to have, for any nonprincipal ultrafilter $\mathcal{U}$ over $\mathbb{N}$, relative commutant in the ultrapower $A^{\prime} \cap A^{\mathcal{U}}$ of density character at least $\mathfrak{c}$. For convenience, we isolate the following notion.

Definition 5.4. A $\mathrm{C}^{*}$-algebra $A$ has many asymptotically central elements if there exists $\delta>0$ such that, for every $n \in \mathbb{N}$, finite subset $F$ of $A$, and $\varepsilon>0$, there exist $a_{1}, \ldots, a_{n}$ in $A$ of norm at most 1 such that, for every $1 \leq i<j \leq n$ and $b \in F,\left\|a_{i}-a_{j}\right\| \geq \delta$ and $\left\|a_{i} b-b a_{i}\right\| \leq \varepsilon$.

Thus Proposition 5.2 gives the following.

Proposition 5.5. Suppose that $A$ is a separable $C^{*}$-algebra that has many asymptotically central elements, and $\mathcal{U}$ is a nonprincipal ultrafilter over $\mathbb{N}$. Then $A^{\prime} \cap A^{\mathcal{U}}$ and $A^{\mathcal{U}}$ both have density character and cardinality equal to $\mathfrak{c}$.

Proof. We have already observed above that $A^{\mathcal{U}}$ has density character at most $\mathfrak{c}$. In order to see that $A^{\prime} \cap A^{\mathcal{U}}$ has density character at least $\mathfrak{c}$, one can apply Proposition 5.2 to the language $L^{\mathrm{C}^{*}}(A)$, the sequence $\left(M_{n}\right)_{n \in \mathbb{N}}$ constantly equal to $A$, and the relative commutant type $\mathfrak{t}(x)$ consisting of the conditions $\|x a-a x\| \leq 0$ for $a \in A$ of norm at most 1 .

This shows that $A^{\prime} \cap A^{\mathcal{U}}$ and $A^{\mathcal{U}}$ both have density character c. Fix a dense subset $E$ of $A^{\mathcal{U}}$ of size c. Observe that, since $E$ is dense, the cardinality of $A^{\mathcal{U}}$ is bounded by the cardinality of the set of sequences of elements of $E$, which is still equal to $\mathfrak{c}$. Therefore $A^{\mathcal{U}}$ has cardinality at most $\mathfrak{c}$, concluding the proof. 
Due to Proposition 5.5, our original question on the size of ultrapowers and relative commutants leads us to consider which $\mathrm{C}^{*}$-algebras have many asymptotically central elements. As it turns out, every infinitedimensional $\mathrm{C}^{*}$-algebra has many asymptotically central elements, as we will show below. We begin with the abelian case. Recall that we are assuming all $\mathrm{C}^{*}$-algebras to be unital.

Lemma 5.6. Suppose that $A$ is an abelian infinite-dimensional $\mathrm{C}^{*}$-algebra. Then $A$ has many asymptotically central elements.

Proof. Since $A$ is abelian, $A$ is isomorphic to the algebra $C(X)$ of continuous complex-valued function over a compact Hausdorff space $X$. Since $A$ is infinite-dimensional, $X$ is not finite. Thus, by normality of $X$ we can find, for every $n \in \mathbb{N}$, positive elements $a_{1}, \ldots, a_{n}$ of $C(X)$ of norm 1 with disjoint supports. (The support of a function $a: X \rightarrow[0,1]$ is the set $\{t \in X: a(t) \neq 0\}$.) This guarantees that $\left\|a_{i}-a_{j}\right\|=1$ for every $1 \leq i<j \leq n$. This concludes the proof.

We now consider the class of continuous trace $\mathrm{C}^{*}$-algebras [69]. These are the $\mathrm{C}^{*}$-algebras that can be realized as the algebras of sections of a bundle over a compact Hausdorff space $X$, with finite-dimensional $\mathrm{C}^{*}$-algebras as fibers. Clearly, commutative $\mathrm{C}^{*}$-algebras correspond to the case of bundles with 1-dimensional fibers.

Lemma 5.7. Suppose that $A$ is an infinite-dimensional continuous trace $\mathrm{C}^{*}$-algebra. Then $A$ has many asymptotically central elements.

Proof. As noted above, $A$ is the algebra of sections of a bundle of over a compact Hausdorff space $X$, with finite-dimensional $C^{*}$-algebras as fibers. Since $A$ is infinite-dimensional, $X$ is infinite. Clearly, the abelian $\mathrm{C}^{*}$-algebra $C(X)$ is isomorphic to a $\mathrm{C}^{*}$-subalgebra of the center $A^{\prime} \cap A$ of $A$. This implies that, since $C(X)$ has many asymptotically central elements, so does $A$.

We consider now the case when $A$ does not have continuous trace.

Lemma 5.8. Let $A$ be a separable $\mathrm{C}^{*}$-algebra that does not have continuous trace. Then $A$ has many asymptotically central elements.

Proof. By [1, Theorem 2.4], there exists a sequence $\left(d_{n}\right)$ of positive elements of norm 1 in $A$ such that, for every $a \in A, \lim _{n \rightarrow+\infty}\left\|d_{n} a-a d_{n}\right\|=0$ and $\delta_{a}:=\lim \sup _{n \rightarrow+\infty}\left\|d_{n}-a\right\|>0$. Consider then the $L^{\mathrm{C}^{*}}(A)$-type $\mathfrak{t}(x)$ consisting of the conditions $\|x a-a x\| \leq 0$ and $\|x-a\| \geq \delta_{a}$ for $a \in A$ and $|1-\|x\|| \leq 0$. Then the type $\mathfrak{t}(x)$ is approximately realized in $A$, and hence it is realized in $A^{\mathcal{U}}$ by countable saturation. We identify $A$ with a subalgebra of $A^{\prime} \cap A^{\mathcal{U}}$. If $\boldsymbol{a}$ is a realization of $\mathfrak{t}(x)$ in $A^{\mathcal{U}}$, then $\boldsymbol{a}$ is an element of norm 1 of $A^{\prime} \cap A^{\mathcal{U}}$ that does not belong to $A$. By completeness of $A$, there exists $\delta>0$ such that $\|\boldsymbol{a}-b\|>\delta$ for every $b \in A$ of norm 1 .

Fix an enumeration $\left(b_{n}\right)$ of a dense subset of the unit ball of $A$. If $\left(a_{n}\right)$ is a representative sequence of $\boldsymbol{a}$, then using Łos' theorem one can recursively define an increasing sequence $\left(n_{k}\right)$ in $\mathbb{N}$ such that, for every $k<m$, $\left\|a_{k}-a_{m}\right\|>\delta$ and $\left\|b_{m} a_{k}-a_{k} b_{m}\right\|<2^{-k}$. This shows that $A$ has many asymptotically central elements.

Lemma 5.7 and Lemma 5.8 together show that every infinite-dimensional $\mathrm{C}^{*}$-algebra has many asymptotically central elements in the sense of Definition 5.4. (Obviously, the converse holds as well.) Combining this with Proposition 5.5 and Theorem 5.1, we finally obtain the following.

Theorem 5.9. Suppose that $A$ is a separable infinite-dimensional $C^{*}$-algebra. If $\mathcal{U}$ is a nonprincipal ultrafilter over $\mathbb{N}$, then the ultrapower $A^{\mathcal{U}}$ and the relative commutant $A^{\prime} \cap A^{\mathcal{U}}$ have density character $\mathfrak{c}$. If the Continuum Hypothesis holds, and $\mathcal{U}, \mathcal{V}$ are nonprincipal ultrafilters over $\mathbb{N}$, then $A^{\mathcal{U}} \cong A^{\mathcal{V}}$ and $A^{\prime} \cap A^{\mathcal{U}} \cong A^{\prime} \cap A^{\mathcal{V}}$.

Proof. The first assertion is an immediate consequence of Proposition 5.5 and the observations above. Suppose now that the Continuum Hypothesis holds. If $\mathcal{U}, \mathcal{V}$ are nonprincipal ultrafilters over $\mathbb{N}$, then $A^{\mathcal{U}}$ and $A^{\mathcal{V}}$ have density character $\aleph_{1}$, they are elementarily equivalent by Łos' theorem, and they are countable saturated by Proposition 4.7. Therefore Theorem 5.1 implies that $A^{\mathcal{U}}$ and $A^{\mathcal{V}}$ are isomorphic. Furthermore, since the diagonal embedding of $A$ in both $A^{\mathcal{U}}$ and $A^{\mathcal{V}}$ is elementary by Los' theorem, again by Theorem 5.1 there exists an isomorphism $\Phi: A^{\mathcal{U}} \rightarrow A^{\mathcal{V}}$ which is the identity on $A$ (canonically identified with a $\mathrm{C}^{*}$-subalgebra of $A^{\mathcal{U}}$ and $A^{\mathcal{V}}$ ). Henceforth, $\Phi$ restricts to an isomorphism from $A^{\prime} \cap A^{\mathcal{U}}$ onto $A^{\prime} \cap A^{\mathcal{V}}$. This concludes the proof.

\section{Strongly SELF-AbSorbing $\mathrm{C}^{*}$-Algebras}

The class of strongly self-absorbing $\mathrm{C}^{*}$-algebras, initially introduced by Toms and Winter in [82], has played in recent years a pivotal role in the study of structure and classification of simple nuclear $\mathrm{C}^{*}$-algebras. In the rest of this section, we want to present some model-theoretic results concerning these algebras, their ultrapowers and relative commutants, obtained in [37]. 
Recall that a separable $\mathrm{C}^{*}$-algebra $D$ has approximately inner half-flip if the canonical embeddings $D \rightarrow D \otimes D$ defined by $d \mapsto d \otimes 1_{D}$ and $d \mapsto 1_{D} \otimes d$ are approximately unitarily equivalent. (Here and in the following, we consider the minimal tensor product of $\mathrm{C}^{*}$-algebras; see [13, Section II.9].) In other words, there exists a sequence $\left(u_{n}\right)$ of unitary elements of $D \otimes D$ such that $\left\|u_{n}\left(d \otimes 1_{D}\right)-\left(d \otimes 1_{D}\right) u_{n}\right\| \rightarrow 0$ for $n \rightarrow+\infty$ for every $d \in D$. A separable $\mathrm{C}^{*}$-algebra $D$ is strongly self-absorbing if it is not isomorphic to $\mathbb{C}$, and the canonical embedding $\operatorname{id}_{D} \otimes 1: D \rightarrow D \otimes D, d \mapsto d \otimes 1_{D}$ is approximately unitarily equivalent to an isomorphism $D \cong D \otimes D$. This condition is very restrictive, indeed a strongly self-absorbing $\mathrm{C}^{*}$-algebra $D$ is automatically simple and nuclear, has approximately inner-half flip, and it is isomorphic to the infinite tensor product $D^{\otimes \mathbb{N}}$. The only currently known examples of strongly self-absorbing $\mathrm{C}^{*}$-algebras are the infinite type uniformly hyperfinite (UHF) $\mathrm{C}^{*}$-algebras [50], the Cuntz algebras $\mathcal{O}_{2}$ and $\mathcal{O}_{\infty}[24]$, the Jiang-Su algebra $\mathcal{Z}$ [58], and their tensor products. In the following, all the strongly self-absorbing $\mathrm{C}^{*}$-algebras are assumed to be separable.

Suppose that $D$ is a strongly self-absorbing $\mathrm{C}^{*}$-algebra, and $A$ is a separable $\mathrm{C}^{*}$-algebra. One says that $A$ is tensorially $D$-absorbing, or simply $D$-absorbing, if $D \otimes A$ is isomorphic to $A$. The notion of $D$-absorption plays a crucial role in the current study of nuclear $\mathrm{C}^{*}$-algebras. The goal of the next subsection is to present a proof of a well-known characterization of $D$-absorption for separable $\mathrm{C}^{*}$-algebras in terms of the notion of positive existential embedding.

6.1. A criterion for $D$-absorption. Throughout this section, we let $D$ be a fixed strongly self-absorbing $\mathrm{C}^{*}$-algebra, and $A, B$ be separable $\mathrm{C}^{*}$-algebras. In the following lemma, we consider objects which are triples $(A, \hat{A}, \eta)$ of two $C^{*}$-algebras $A, \hat{A}$ together with an embedding $\eta: A \rightarrow \hat{A}$. These can be regarded as structures in a two-sorted language $\mathcal{L}$ which has sorts for the $\mathrm{C}^{*}$-algebras $A, \hat{A}$, function and relation symbols for the $\mathrm{C}^{*}$-algebra structure on $A, \hat{A}$, and a function symbol for the embedding $\eta: A \rightarrow \hat{A}$.

Lemma 6.1. Suppose that $A, B$ are $\mathrm{C}^{*}$-algebras and $\Phi: A \rightarrow B$ is a positively existential embedding. If $C$ is a nuclear $\mathrm{C}^{*}$-algebra, then $\operatorname{id}_{C} \otimes \Phi: C \otimes A \rightarrow C \otimes B, c \otimes a \mapsto c \otimes \Phi(a)$ is a positively existential embedding. Furthermore, the pair $\left(\Phi, \operatorname{id}_{C} \otimes \Phi\right)$ defines a positive existential embedding from $\left(A, C \otimes A, 1_{C} \otimes \mathrm{id}_{A}\right)$ to $\left(B, C \otimes B, 1_{C} \otimes \mathrm{id}_{B}\right)$ regarded as $\mathcal{L}$-structures.

Proof. We will use below that, since $C$ is nuclear, maximal and minimal tensor products with $C$ coincide.

Fix a countably incomplete ultrafilter $\mathcal{U}$. Since $\Phi$ is a positively existential embedding, there exists a morphism $\Psi: B \rightarrow A^{\mathcal{U}}$ such that $\Psi \circ \Phi=\Delta_{A}$. One can then consider $\operatorname{id}_{C} \otimes \Psi: C \otimes B \rightarrow C \otimes A^{\mathcal{U}}$. Since $C$ is nuclear, the tensor product $C \otimes A^{\mathcal{U}}$ can be identified with the maximal tensor product. Let $\eta: C \otimes A^{\mathcal{U}} \rightarrow(C \otimes A)^{\mathcal{U}}$ be the canonical morphism obtained via the universal property of the maximal tensor product from the morphisms with commuting ranges $\Delta_{C}: C \rightarrow C^{\mathcal{U}} \subset(C \otimes A)^{\mathcal{U}}$ and $\operatorname{id}_{A^{\mathcal{U}}}: A^{\mathcal{U}} \rightarrow A^{\mathcal{U}} \subset(C \otimes A)^{\mathcal{U}}$. Observe that

$$
\eta \circ\left(\operatorname{id}_{C} \otimes \Psi\right) \circ\left(\operatorname{id}_{C} \otimes \Phi\right)=\eta \circ\left(\operatorname{id}_{C} \otimes \Delta_{A}\right)=\operatorname{id}_{C \otimes A}
$$

Thus $\eta \circ\left(\operatorname{id}_{C} \otimes \Psi\right)$ witnesses that $\mathrm{id}_{C} \otimes \Phi$ is positively existential.

Consider now $\left(A, C \otimes A, \operatorname{id}_{C} \otimes A\right)$ and $\left(B, C \otimes B, \operatorname{id}_{C} \otimes B\right)$ as $\mathcal{L}$-structures, and observe that the pair $\left(\Phi, \operatorname{id}_{C} \otimes \Phi\right)$ defines an $\mathcal{L}$-morphism between such $\mathcal{L}$-structures. Observe also that $\left(A^{\mathcal{U}},(C \otimes A)^{\mathcal{U}},\left(1_{C} \otimes \mathrm{id}_{A}\right)^{\mathcal{U}}\right)$ can be regarded as the ultrapower of $\left(A, C \otimes A, 1_{C} \otimes \mathrm{id}_{A}\right)$ as an $\mathcal{L}$-structure. Furthermore, the pair $\left(\Psi, \eta \circ\left(\operatorname{id}_{C} \otimes \Psi\right)\right)$ defines an $\mathcal{L}$-morphism from $\left(B, C \otimes B, 1_{C} \otimes \mathrm{id}_{B}\right)$ to $\left(A^{\mathcal{U}},(C \otimes A)^{\mathcal{U}},\left(1_{C} \otimes \mathrm{id}_{A}\right)^{\mathcal{U}}\right)$ whose composition with $\left(\Phi, \operatorname{id}_{C} \otimes \Phi\right)$ is the canonical embedding of $\left(A, C \otimes A, \operatorname{id}_{C} \otimes A\right)$ into its ultrapower. This witnesses that the pair $\left(\Phi, \mathrm{id}_{C} \otimes \Phi\right)$ is a positively existential embedding from $\left(A, C \otimes A, 1_{C} \otimes \mathrm{id}_{A}\right)$ to $\left(B, C \otimes B, 1_{C} \otimes \mathrm{id}_{B}\right) \operatorname{re}_{-}$ garded as $\mathcal{L}$-structures.

The following fundamental intertwining lemma is [70, Proposition 2.3.5].

Lemma 6.2. Suppose that $\Phi: A \rightarrow B$ is an embedding. Assume that for every $\varepsilon>0$ and for every finite subset $F_{A}$ of $A$ and $F_{B}$ of $B$ there exists a unitary element $z$ of $B$ such that

(1) $\left\|z \Phi(a) z^{*}-\Phi(a)\right\| \leq \varepsilon$ for $a \in F_{A}$, and

(2) $\inf _{x \in A}\left\|z^{*} b z-\Phi(x)\right\| \leq \varepsilon$ for $b \in F_{B}$.

Then $\Phi$ is approximately unitarily equivalent to an isomorphism $\Psi$.

Using Lemma 6.2 one can obtain the following.

Lemma 6.3. If the canonical embedding $1_{D} \otimes \mathrm{id}_{A}: A \rightarrow D \otimes A$ is positively existential, then it is approximately unitarily equivalent to an isomorphism. 
Proof. Fix a finite subset $F$ of $A$, a finite subset $F^{\prime}$ of $D \otimes A$, and $\varepsilon>0$. Consider the structure

$$
\mathcal{A}=\left(A, D \otimes A, 1_{D} \otimes \operatorname{id}_{A}: A \rightarrow D \otimes A\right)
$$

and the structure

$$
\mathcal{B}=\left(D \otimes A, D \otimes D \otimes A, 1_{D} \otimes \operatorname{id}_{D \otimes A}: D \otimes A \rightarrow D \otimes D \otimes A\right) .
$$

One can naturally consider $\mathcal{A}$ and $\mathcal{B}$ as $\mathcal{L}$-structures, where $\mathcal{L}$ is the multi-sorted language considered in Lemma 6.1. We are assuming that the embedding

$$
1_{D} \otimes \mathrm{id}_{A}: A \rightarrow D \otimes A
$$

is positively existential. Therefore by Lemma 6.1 the embedding $\operatorname{id}_{D} \otimes 1_{D} \otimes \mathrm{id}_{A}: D \otimes A \rightarrow D \otimes D \otimes A$ is positively existential. Furthermore, the pair $\Psi=\left(1_{D} \otimes \mathrm{id}_{A}, \mathrm{id}_{D} \otimes 1_{D} \otimes \mathrm{id}_{A}\right)$ defines an existential embedding from $\mathcal{A}$ to $\mathcal{B}$ regarded as $\mathcal{L}$-structures.

Since $D$ has approximately inner half-flip, there exists a unitary element $v$ of $D \otimes D$ such that, if $\hat{u}:=v \otimes 1 \in$ $D \otimes D \otimes A$, then

(1) $\left\|\hat{u}\left(1_{D} \otimes 1_{D} \otimes a\right) \hat{u}^{*}-\left(1_{D} \otimes 1_{D} \otimes a\right)\right\|<\varepsilon$ for $a \in F$, and

(2) $\operatorname{dist}\left(\hat{u}^{*}\left(\mathrm{id}_{D} \otimes 1_{D} \otimes \mathrm{id}_{A}\right)(b) \hat{u}, 1_{D} \otimes D \otimes A\right)<\varepsilon$ for $b \in F^{\prime}$.

Using the fact that the $\mathcal{L}$-morphism $\Psi: \mathcal{A} \rightarrow \mathcal{B}$ is positively $\mathcal{L}$-existential and that the unitary group is a positively existentially definable set, one can conclude that there exists a unitary element $u$ of $D \otimes A$ such that

(1) $\left\|u\left(1_{D} \otimes a\right) u^{*}-\left(1_{D} \otimes a\right)\right\|<\varepsilon$ for $a \in F$, and

(2) $\operatorname{dist}\left(u^{*} b u, 1_{D} \otimes A\right)<\varepsilon$ for $b \in F^{\prime}$.

This witnesses that $1_{D} \otimes \operatorname{id}_{A}:(A, \alpha) \rightarrow\left(D \otimes A, \operatorname{id}_{D} \otimes \alpha\right)$ satisfies the assumptions of Lemma 6.2.

Lemma 6.4. The embedding

is positively existential.

$$
1_{D} \otimes \operatorname{id}_{D \otimes A}: D \otimes A \rightarrow D \otimes D \otimes A
$$

Proof. Since $D$ is strongly self-absorbing, it is enough to show that the embedding

$$
1_{D} \otimes \operatorname{id}_{D \otimes \mathbb{N}} \otimes A: D^{\otimes \mathbb{N}} \otimes A \rightarrow D \otimes D^{\otimes \mathbb{N}} \otimes A
$$

is positively existential. For every $n \in \mathbb{N}$, let

$$
\Psi_{n}: D \rightarrow D^{\otimes \mathbb{N}} \otimes A
$$

be the embedding induced by the embedding of $D$ into $D^{\otimes \mathbb{N}}$ as $n$-th tensor factor. Suppose that $\varphi\left(x_{1}, \ldots, x_{\ell}\right)$ is a positive existential $L^{\mathrm{C}^{*}}(A)$-formula, $\varepsilon>0, r \in \mathbb{R}$, and consider the condition $\varphi\left(x_{1}, \ldots, x_{\ell}\right) \leq r$. Assume that $\sum_{k} d_{i, k} \otimes a_{i, k}$ for $i=1,2, \ldots, \ell$ is an $\ell$-tuple in $D \otimes D^{\otimes \mathbb{N}} \otimes A$ satisfying the condition $\varphi\left(x_{1}, \ldots, x_{\ell}\right)<r$, where $d_{i, k} \in D$ and $a_{i, k} \in D^{\otimes \mathbb{N}} \otimes A$. Then, for $n \in \mathbb{N}$ large enough, $\sum_{k} \Psi_{n}\left(d_{i, k}\right) a_{i, k}$ for $i=1,2, \ldots, \ell$ is an $\ell$-tuple in $D^{\otimes \mathbb{N}} \otimes A$ satisfying the condition $\varphi\left(x_{1}, \ldots, x_{\ell}\right) \leq r+\varepsilon$. This concludes the proof that $1_{D} \otimes \mathrm{id}_{D \otimes \mathbb{N}} \otimes A$ is a positively existential embedding.

Using the results above, we can give a characterization of $D$-absorption.

Theorem 6.5. Suppose that $A$ is a separable $C^{*}$-algebra, and $D$ is a separable strongly self-absorbing $C^{*}$-algebra. Let $\mathcal{U}$ be a countably incomplete ultrafilter. The following statements are equivalent:

(1) $A$ is D-absorbing;

(2) the embedding $\operatorname{id}_{A} \otimes 1_{D}: A \rightarrow A \otimes D$ is positively existential;

(3) the embedding $\operatorname{id}_{A} \otimes 1_{D}: A \rightarrow A \otimes D$ is approximately unitarily equivalent to an isomorphisms;

(4) $D$ embeds into $A^{\prime} \cap A^{\mathcal{U}}$;

(5) if $\mathfrak{t}(\bar{x})$ is a positive quantifier-free type approximately realized in $D$, then the type $\mathfrak{t}(\bar{x}) \cup\left\{\left\|x_{i} a-a x_{i}\right\| \leq 0: a \in A\right\}$ is approximately realized in $A$.

Proof. The implication $(1) \Rightarrow(2)$ is a consequence of Lemma 6.4 , while the implication $(2) \Rightarrow(3)$ is a consequence of Lemma 6.3. The equivalence $(2) \Leftrightarrow(4)$ is a consequence of Lemma 4.14 , observing that $D$ is simple so a morphism $D \rightarrow A^{\prime} \cap A^{\mathcal{U}}$ is necessarily an embedding, while the equivalence $(4) \Leftrightarrow(5)$ follows from Eos' theorem and countable saturation of ultrapowers. Finally, the implication $(3) \Rightarrow(1)$ is obvious.

Theorem 6.5 motivates the following definition.

Definition 6.6. A (not necessarily separable) $\mathrm{C}^{*}$-algebra $A$ is $D$-absorbing if it satisfies Condition (5) in Theorem 6.5. 
In view of Theorem 6.5, this definition is consistent with the usual one in the case of separable $\mathrm{C}^{*}$-algebras. Furthermore, when $A$ is positively quantifier-free saturated, $A$ is $D$-absorbing if and only if, for every separable subalgebra $S$ of $A, D$ embeds into $S^{\prime} \cap A$. This shows that $S^{\prime} \cap D$ is still $D$-absorbing and positively quantifier-free saturated

It is clear from the definition that the property of being $D$-absorbing is axiomatizable. Indeed, this is witnessed by the conditions

$$
\sup _{x_{1}, \ldots, x_{n}} \inf _{y_{1}, \ldots, y_{n}} \max \left\{\varphi\left(x_{1}, \ldots, x_{n}\right),\left\|x_{i} y_{j}-y_{j} x_{i}\right\|: 1 \leq i, j \leq n\right\} \leq 0
$$

where $\varphi(\bar{x})$ varies among the positive quantifier-free formulas for which the condition $\varphi(\bar{x}) \leq 0$ is realized in $D$.

Definition 6.7. A (positive) supinf-formula is a formula of the form $\sup _{\bar{x}} \inf _{\bar{y}} \psi(\bar{x}, \bar{y})$ where $\psi(\bar{x}, \bar{y})$ is a (positive) quantifier-free formula. A class is (positively) sup inf-axiomatizable if it is axiomatizable, as witnessed by conditions of the form $\varphi \leq r$, where $\varphi$ is a (positive) sup inf-formula.

The above argument shows that the class of $D$-absorbing $\mathrm{C}^{*}$-algebras is sup inf-axiomatizable.

6.2. Relative commutants of $D$-absorbing $\mathbf{C}^{*}$-algebras. Suppose as above that $D$ is a strongly selfabsorbing $\mathrm{C}^{*}$-algebra. Let now $C$ be a (not necessarily separable) $D$-absorbing $\mathrm{C}^{*}$-algebra, in the sense just defined. Recall that two morphisms $\Phi_{1}, \Phi_{2}: A \rightarrow B$ are unitarily equivalent if there exists a unitary element $u$ of $B$ such that $\Phi_{2}=\operatorname{Ad}(u) \circ \Phi_{1}$. In the following theorem, we denote by $\aleph_{1}$ the first uncountable cardinal number.

Theorem 6.8. Let $D$ be a strongly self-absorbing $C^{*}$-algebra. Suppose that $\theta: D \rightarrow C$ is an embedding, and that $C$ is D-absorbing and positively quantifier-free countably saturated. The following assertions hold:

(1) Any two embeddings $D \rightarrow C$ are unitarily equivalent;

(2) For every separable $C^{*}$-subalgebra $A$ of $\theta(D)^{\prime} \cap C$ and every separable $C^{*}$-subalgebra $B$ of $C$ there exists a unitary $u \in A^{\prime} \cap C$ such that $u B u^{*} \subset \theta(D)^{\prime} \cap C$;

(3) $\theta(D)^{\prime} \cap C$ is an elementary substructure of $C$;

(4) If $C$ has density character $\aleph_{1}$, then the inclusion $\theta(D)^{\prime} \cap C \hookrightarrow C$ is approximately unitarily equivalent to an isomorphism;

(5) If $C$ is countably saturated, then $\theta(D)^{\prime} \cap C$ is countably saturated.

Proof. Since $C$ is $D$-absorbing and positively quantifier-free saturated, for every separable $\mathrm{C}^{*}$-subalgebra $S$ of $C$ one has that $S^{\prime} \cap C$ is also $D$-absorbing and positively quantifier-free saturated.

(1): Let $\theta_{1}: D \rightarrow C$ be an embedding. We will show that $\theta_{1}$ is unitarily equivalent to $\theta$. Let us initially assume that the ranges of $\theta$ and $\theta_{1}$ commute. We can choose a sequence $\left(u_{n}\right)$ of unitaries in $D$ witnessing the fact that $D$ has approximately inner half-flip. Define $\Theta: D \otimes D \rightarrow C$ by $d_{0} \otimes d_{1} \mapsto \theta\left(d_{0}\right) \theta_{1}\left(d_{1}\right)$. Considering the unitaries $\Theta\left(u_{n}\right)$ for $n \in \mathbb{N}$ and applying the fact that $C$ is positively quantifier-free countably saturated, we conclude that there exists a unitary $u \in A$ such that $u \theta(d)=u \Theta(d \otimes 1)=\Theta(1 \otimes d) u=\theta_{1}(d) u$. This shows that $\theta, \theta_{1}$ are unitarily equivalent.

In the general case, when the ranges of $\theta, \theta_{1}$ do not necessarily commute, since $D$ is strongly self-absorbing and $C$ is positively quantifier-free countably saturated, we can find an embedding $\theta_{2}: D \rightarrow C$ whose range commutes with the ranges of $\theta$ and $\theta_{1}$. Therefore by the above we have that $\theta$ is unitarily equivalent to $\theta_{2}$ and $\theta_{1}$ is unitarily equivalent to $\theta_{2}$. Hence, $\theta$ is unitarily equivalent to $\theta_{1}$.

For the rest of the proof, we identify $D$ with its image under $\theta$ inside $C$.

(2): Fix a separable $C^{*}$-subalgebra $A$ of $D^{\prime} \cap C$ and a separable $\mathrm{C}^{*}$-subalgebra $B$ of $C$. We want to show that there exists $u \in A^{\prime} \cap C$ such that $u B u^{*} \subset D^{\prime} \cap C$.

Since $C$ is $D$-absorbing and positively quantifier-free saturated, the same holds for $A^{\prime} \cap C$ and $A^{\prime} \cap B^{\prime} \cap C$. Thus we can fix an embedding $\Psi: D \rightarrow A^{\prime} \cap B^{\prime} \cap C$. By (1) applied to the pair of embeddings $D \rightarrow A^{\prime} \cap C$ given by $\Psi$ and the inclusion $\iota: D \subset A^{\prime} \cap C$, there exists a unitary $u \in A^{\prime} \cap C$ such that $\Psi=\operatorname{Ad}\left(u^{*}\right) \circ \iota$ or, equivalently, $u^{*} d u=\Psi(d)$ for every $d \in D$. Therefore, if $b \in B$ and $d \in D$, we have that

$$
\left\|u b u^{*} d-d u b u^{*}\right\|=\left\|b u^{*} d u-u^{*} d u b\right\|=\|b \Psi(d)-\Psi(d) b\|=0 .
$$

This concludes the proof.

(3): By the Tarski-Vaught test (Proposition 4.11), it suffices to show that if $r \in \mathbb{R}, \varphi(\bar{x}, \bar{y})$ is a formula, $\bar{a}$ is a tuple in $D^{\prime} \cap C$, and $\bar{b}$ is a tuple in $C$ such that $\varphi^{C}(\bar{a}, \bar{b})<r$, then there exists a tuple $\bar{d}$ in $D^{\prime} \cap C$ such that $\varphi^{C}(\bar{a}, \bar{d})<r$. Let $A$ be the $\mathrm{C}^{*}$-subalgebra of $D^{\prime} \cap C$ generated by $\bar{a}$, and let $B$ be the $\mathrm{C}^{*}$-subalgebra of 
$C$ generated by $\bar{b}$. Then by (2) there exists a unitary $u \in A^{\prime} \cap C$ such that $u B u^{*} \subset D^{\prime} \cap C$. Thus we have that $\bar{d}:=u \bar{b} u^{*}$ is a tuple in $D^{\prime} \cap C$ such that

$$
\varphi^{C}(\bar{a}, \bar{d})=\varphi^{C}\left(u \bar{a} u^{*}, u \bar{b} u^{*}\right)=\varphi^{C}(\bar{a}, \bar{b})<r,
$$

concluding the proof.

(4): Suppose now that $C$ has density character $\aleph_{1}$. Thus we can fix an enumeration $\left(b_{\lambda}\right)_{\lambda<\omega_{1}}$ of a subset of $C$ such that, for every $\mu<\lambda,\left\{b_{\lambda}: \mu<\lambda<\omega_{1}\right\}$ is a dense subset of $C$. Similarly, we can fix an enumeration $\left(a_{\lambda}\right)_{\lambda<\omega_{1}}$ of a subset of $D^{\prime} \cap C$ such that, for every $\mu<\lambda,\left\{a_{\lambda}: \mu<\lambda<\omega_{1}\right\}$ is a dense subset of $D^{\prime} \cap C$. Here, $\omega_{1}$ denotes the first uncountable ordinal. We will define by transfinite recursion elements $\hat{a}_{\lambda}$ of $D^{\prime} \cap C$, elements $\hat{b}_{\lambda}$ of $C$, and unitaries $u_{\lambda}$ in $C$ for $\lambda<\omega_{1}$ such that, for every $\mu \leq \lambda<\omega_{1}$,

$$
u_{\lambda} b_{\mu} u_{\lambda}^{*}=\hat{a}_{\mu} \quad \text { and } \quad u_{\lambda} \hat{b}_{\mu} u_{\lambda}^{*}=a_{\mu} .
$$

Granted the construction, we can define the map $\Phi:\left\{a_{\mu}: \mu<\omega_{1}\right\} \rightarrow C$ by setting $\Phi\left(a_{\mu}\right)=\hat{b}_{\mu}$. Then by construction, $\Phi$ is approximately unitarily equivalent to the inclusion map of $\left\{a_{\mu}: \mu<\omega_{1}\right\}$ inside $C$. Therefore $\Phi$ extends to a *-homomorphism $\Phi: D^{\prime} \cap C \rightarrow C$, which is approximately unitarily equivalent to the inclusion map $D^{\prime} \cap C \rightarrow C$. It remains to show that $\Phi$ is onto. Fix $\varepsilon>0$ and $\mu<\omega_{1}$. Then there exists an ordinal $\mu<\lambda<\omega_{1}$ such that $\left\|\hat{a}_{\mu}-a_{\lambda}\right\|<\varepsilon$. Thus we have that

$$
\left\|\Phi\left(\hat{a}_{\mu}\right)-b_{\mu}\right\| \leq \varepsilon+\left\|\Phi\left(a_{\lambda}\right)-u_{\lambda}^{*} \hat{a}_{\mu} u_{\lambda}\right\| \leq 2 \varepsilon+\left\|\hat{b}_{\lambda}-u_{\lambda}^{*} a_{\lambda} u_{\lambda}\right\|=2 \varepsilon .
$$

Since this holds for every $\mu<\omega_{1}$ and for every $\varepsilon>0$, this shows that $\Phi$ is onto.

It remains to describe the recursive construction. Suppose that the elements $\hat{a}_{\mu}, \hat{b}_{\mu}, u_{\mu}$ have been constructed for $\mu<\lambda$. Define $A$ to be the separable $\mathrm{C}^{*}$-subalgebra of $D^{\prime} \cap C$ generated by $\hat{a}_{\mu}, a_{\mu}$ for $\mu<\lambda$. Let also $B$ be the separable $\mathrm{C}^{*}$-subalgebra of $C$ generated by $a_{\mu}, b_{\mu}, \hat{a}_{\mu}, \hat{b}_{\mu}$ for $\mu<\lambda$.

Suppose initially that $\lambda$ is a successor ordinal. We let $\lambda-1$ be the immediate predecessor of $\lambda$. Then by (2) there exists a unitary $v \in A^{\prime} \cap C$ such that $v u_{\lambda-1} b_{\lambda} u_{\lambda-1}^{*} v^{*} \in D^{\prime} \cap C$. Set then $u_{\lambda}:=v u_{\lambda-1}, \hat{a}_{\lambda}:=u_{\lambda} b_{\lambda} u_{\lambda}^{*} \in D^{\prime} \cap C$ and $\hat{b}_{\lambda}=u_{\lambda}^{*} a_{\lambda} u_{\lambda}$. Now we have that, by definition

$$
u_{\lambda} b_{\lambda} u_{\lambda}^{*}=\hat{a}_{\lambda} \quad \text { and } \quad u_{\lambda} \hat{b}_{\lambda} u_{\lambda}^{*}=a_{\lambda} .
$$

For $\mu<\lambda$ we have that, since $v \in A^{\prime} \cap C$,

$$
u_{\lambda} b_{\mu} u_{\lambda}^{*}=v u_{\lambda-1} b_{\mu} u_{\lambda-1}^{*} v^{*}=v \hat{a}_{\mu} v^{*}=\hat{a}_{\mu}
$$

and

$$
u_{\lambda} \hat{b}_{\mu} u_{\lambda}^{*}=v u_{\lambda-1} \hat{b}_{\mu} u_{\lambda-1}^{*} v^{*}=v a_{\mu} v^{*}=a_{\mu} .
$$

This concludes the construction in the case when $\lambda$ is a successor ordinal. When $\lambda$ is a limit ordinal, one can obtain $u_{\lambda}$ by applying positive quantifier-free countable saturation of $C$ to the positive quantifier-free $L^{\mathrm{C}^{*}}(B)$ type $\mathfrak{t}(x)$ consisting of the conditions $\left\|x^{*} x-1\right\| \leq 0,\left\|x x^{*}-1\right\| \leq 0,\left\|x b_{\mu} x^{*}-\hat{a}_{\mu+1}\right\| \leq 0$ for $\mu<\lambda$, and $\left\|x \hat{b}_{\mu} x^{*}-a_{\mu}\right\| \leq 0$ for $\mu<\lambda$. Such a type is approximately realized in $C$ by the inductive hypothesis that $u_{\mu}$ has been defined for $\mu<\lambda$. Therefore such a type is realized in $C$. One can then let $u_{\lambda}$ be any realization of $\mathfrak{t}(x)$. This concludes the recursive construction.

(5): Suppose that $C$ is countably saturated. Suppose that $A$ is a separable $\mathrm{C}^{*}$-subalgebra of $D^{\prime} \cap C$, and $\mathfrak{t}(\bar{x})$ is an $L^{\mathrm{C}^{*}}(A)$-type which is approximately realized in $D^{\prime} \cap C$. Then $\mathfrak{t}(\bar{x})$ is also approximately realized in $C$. Since by assumption $C$ is countably saturated, $\mathfrak{t}(\bar{x})$ has a realization $\bar{b}$ in $C$. By (2) there exists a unitary $u \in A^{\prime} \cap C$ such that $u \bar{b} u^{*} \subset D^{\prime} \cap C$. Since $u \in A^{\prime} \cap C$, the map $\operatorname{Ad}(u)$ is an $L^{\mathrm{C}^{*}}(A)$-automorphism of $C$. For every condition $\varphi(\bar{x}) \leq r$ in $\mathfrak{t}(\bar{x})$ we have that $\varphi^{C}(\bar{b}) \leq r$, and hence $\varphi^{C}\left(u \bar{b} u^{*}\right) \leq r$. Since $u b u^{*} \in D^{\prime} \cap C$ and $D^{\prime} \cap C$ is an elementary substructure of $C$, we have that $\varphi^{D^{\prime} \cap C}\left(u \bar{b} u^{*}\right) \leq r$. Since this holds for every condition in $\mathfrak{t}(\bar{x}), u \bar{b} u^{*}$ is a realization of $\mathfrak{t}(\bar{x})$ in $D^{\prime} \cap C$. This concludes the proof that $D^{\prime} \cap C$ is countably saturated.

Corollary 6.9. Let $D$ be a strongly self-absorbing $\mathrm{C}^{*}$-algebra, and $A$ is a separable $D$-absorbing $\mathrm{C}^{*}$-algebra. If $\mathcal{U}$ is a countably incomplete ultrafilter, then the conclusions of Theorem 6.8 holds for $C=A^{\mathcal{U}}$ and for $C=A^{\prime} \cap A^{\mathcal{U}}$. In particular, if the Continuum Hypothesis holds, and if $D \subset A$ is a copy of $D$ inside $A$, then $D^{\prime} \cap A^{\mathcal{U}}$ and $A^{\mathcal{U}}$ are isomorphic.

Proof. It suffices to observe that the algebras $A^{\mathcal{U}}$ and $A^{\prime} \cap A^{\mathcal{U}}$ are $D$-absorbing and positively quantifier-free saturated. The ultrapower $A^{\mathcal{U}}$ has density character $\mathfrak{c}$ by 5.9. Since $\mathrm{CH}$ is the assertion that $\mathfrak{c}$ is the first uncountable cardinal $\aleph_{1}, \mathrm{CH}$ implies that $A^{\mathcal{U}}$ has density character $\aleph_{1}$. Thus the last assertion is a consequence of item (4) of Theorem 6.8 . 
6.3. Strongly self-absorbing $\mathbf{C}^{*}$-algebras are existentially closed. Suppose that $\mathcal{C}$ is a class of $\mathrm{C}^{*}$ algebras. Then a $\mathrm{C}^{*}$-algebra $A$ is existentially closed in $\mathcal{C}$ if $A$ belongs to $\mathcal{C}$ and any embedding $\Phi: A \rightarrow B$ of $A$ into a $\mathrm{C}^{*}$-algebra $B$ from $\mathcal{C}, \Phi$ is existential. This means that if $\varphi(\bar{x})$ is an existential formula and $\bar{a}$ is a tuple in $A$, then $\varphi^{A}(\bar{a})=\varphi^{B}(\Phi(\bar{a}))$.

Let now $D$ be a strongly self-absorbing $\mathrm{C}^{*}$-algebra, and consider the class $\mathcal{C}_{D}$ of $\mathrm{C}^{*}$-algebras that embed into an ultrapower of $D$. Observe that $\mathcal{C}_{D}$ is an elementary class. Indeed, a $\mathrm{C}^{*}$-algebra $A$ belongs to $\mathcal{C}_{D}$ if and only if $\varphi^{A} \leq \varphi^{D}$ for every universal sentence $\varphi$. When $D$ is the infinite type UHF algebra $\bigotimes_{n \in \mathbb{N}} M_{n}(\mathbb{C})^{\otimes \mathbb{N}}, \mathcal{C}_{D}$ is the class of MF algebras [14, 20]. The Kirchberg embedding conjecture asserts that, when $D$ is the Cuntz algebra $\mathcal{O}_{2}, \mathcal{C}_{D}$ contains all $\mathrm{C}^{*}$-algebras [55].

Proposition 6.10. Let $D$ be a strongly self-absorbing $C^{*}$-algebra. Then $D$ is existentially closed in $\mathcal{C}_{D}$.

Proof. Suppose that $D \subset B$ where $B \in \mathcal{C}_{D}$. Then we can assume that $B \subset D^{\mathcal{U}}$ for some countably incomplete ultrafilter $\mathcal{U}$. Since all the embeddings of $D$ into $D^{\mathcal{U}}$ are unitarily conjugate, we can assume that the composition of the inclusions $D \subset B$ and $B \subset D^{\mathcal{U}}$ is the diagonal embedding of $D$ into $D^{\mathcal{U}}$.

Fix a quantifier-free formula $\varphi(\bar{x}, \bar{y})$. If $\bar{a}$ is a tuple in $D$ and $\bar{b}$ is a tuple in $B \subset D^{\mathcal{U}}$ such that $\varphi(\bar{a}, \bar{b})<r$, by Eos' theorem there exists a tuple $\bar{d}$ in $D$ such that $\varphi(\bar{a}, \bar{d})<r$. This shows that the inclusion $D \subset B$ is existential.

\section{Model THEORY AND NUCLEAR C*-ALGEBRAS}

7.1. The classification programme. Much of the theory of $\mathrm{C}^{*}$-algebras in the last twenty years has focused on the structure and classification of simple, separable nuclear $\mathrm{C}^{*}$-algebra, in the framework of the Elliott classification programme. Nuclearity, also called amenability, is a regularity property for $\mathrm{C}^{*}$-algebras with several equivalent reformulations. It can naturally be defined in the broader category of operator systems and unital completely positive maps. A linear map $\Phi: A \rightarrow B$ between $\mathrm{C}^{*}$-algebras is unital completely positive (ucp) if $\Phi(1)=1$ and, for every $n \in \mathbb{N}, \operatorname{id}_{M_{n}(\mathbb{C})} \otimes \Phi: M_{n}(\mathbb{C}) \otimes A \rightarrow M_{n}(\mathbb{C}) \otimes B$ maps positive elements to positive elements. A $\mathrm{C}^{*}$-algebra is nuclear if the identity map of $A$ is the pointwise limit of ucp maps of the form $\Psi \circ \Phi: A \rightarrow A$, where $\Phi: A \rightarrow M_{n}(\mathbb{C})$ and $\Psi: M_{n}(\mathbb{C}) \rightarrow A$ are ucp maps, and $n \in \mathbb{N}$. This definition admits several equivalent reformulations, including prominently the following: for any $\mathrm{C}^{*}$-algebra $B$, the maximal and the minimal tensor product norms on the algebraic tensor product of $A$ and $B$ coincide. Thus, the class of $\mathrm{C}^{*}$-algebras is endowed with a single canonical $\mathrm{C}^{*}$-algebraic tensor product.

The first hints that a satisfactory classification of separable, nuclear $\mathrm{C}^{*}$-algebras could be achieved goes back to the seminal works of Glimm [50] and Elliott [30], who classified those separable, nuclear $\mathrm{C}^{*}$-algebras that can be realized as direct limits of finite-dimensional $\mathrm{C}^{*}$-algebras. In the modern perspective, the invariant used in these results is the $K_{0}$-group. This is a countable ordered abelian group with a distinguished order unit. Together with the $K_{1}$-group, it constitutes the $K$-theory of a given $\mathrm{C}^{*}$-algebra. Originally developed in algebraic geometry [4], $K$-theory was then translated into purely algebraic language [71], and then incorporated in the theory of $\mathrm{C}^{*}$-algebras [72].

The class of arbitrary separable, nuclear $\mathrm{C}^{*}$-algebras is extensive, in that it contains all the algebras of the form $C(X)$ where $X$ is a compact metrizable space. For such algebras, isomorphism coincides with homeomorphism of the corresponding space. Since a meaningful classificationof arbitrary compact metrizable spaces is considered out of reach, it is natural to impose restrictions on the class of algebras under consideration to rule out complexity arising from the purely topological setting. One such a natural assumption consists in demanding that the $\mathrm{C}^{*}$-algebras under consideration be simple, i.e. have no nontrivial ideals.

Building on the results of Glimm and Elliott mentioned above, broader classes of simple, separable $\mathrm{C}^{*}$-algebras have been classified in the 1980s and 1990s due to the work of many hands. In this case, the invariant consisted on the K-theory together with the trace simplex. A tracial state on a $\mathrm{C}^{*}$-algebra is a unital linear functional $\tau$ satisfying the trace identity $\tau(x y)=\tau(y x)$. The space $T(A)$ of tracial states over a separable $\mathrm{C}^{*}$-algebra $A$ forms a Choquet simplex, which is called the trace simplex. Traces can be seen as a noncommutative analog of measures, and so the trace simplex encodes the measure-theoretic information on the given $\mathrm{C}^{*}$-algebras. The conjunction of the K-theory of a $\mathrm{C}^{*}$-algebra together with its trace simplex and a canonical pairing between them, is called Elliott invariant.

Motivated by these positive results, Elliott proposed the programme - known as the Elliott classification programme - of classifying simple, separable, nuclear $\mathrm{C}^{*}$-algebras by their Elliott invariant [31]. Despite substantial progress, examples due to Rørdam and Toms showed that, in general, the Elliott invariant is not a complete invariant for simple, separable, nuclear $\mathrm{C}^{*}$-algebras. In [80, 81], nonisomorphic simple separable $\mathrm{C}^{*}$ algebras with the same Elliott invariant have been constructed. The invariant used to distinguish such algebras 
is the radius of comparison. Interestingly, such an invariant is captured by the first-order theory of a $\mathrm{C}^{*}$-algebra [36, Section 8.4]. In fact, no example of not elementarily equivalent simple, separable, nuclear $\mathrm{C}^{*}$-algebras with the same Elliott invariant is currently known. This motivated the following problem, asked in [36].

Problem 7.1. Is the Elliott invariant together with the first-order theory a complete invariant for simple, separable, nuclear $\mathrm{C}^{*}$-algebras?

In a different direction, the counterexamples due Rørdam and Toms have suggested to restrict the scope of the Elliott classification programme to a suitable class of "well-behaved" simple, separable, nuclear $\mathrm{C}^{*}$-algebras. One interpretation of what well-behaved should mean for a simple, separable, nuclear $\mathrm{C}^{*}$-algebra is to absorb tensorially the Jiang-Su algebra $\mathcal{Z}$. This is a strongly self-absorbing $\mathrm{C}^{*}$-algebra, and in fact the unique strongly self-absorbing $\mathrm{C}^{*}$-algebra that embeds into any other self-absorbing $\mathrm{C}^{*}$-algebra. The property of $\mathcal{Z}$-absorption is conjecturally equivalent for simple, separable, nuclear $\mathrm{C}^{*}$-algebras to other regularity properties of very different nature (topological, cohomological). This is the subject of the Toms-Winter conjecture, which has been so far verified in many cases, prominently including the case when the trace simplex is itself well-behaved (it has closed extreme boundary).

The revised Elliott classification programme for $\mathcal{Z}$-absorbing simple, separable, nuclear $\mathrm{C}^{*}$-algebras has recently been completed, due to the work of many authors, modulo the standing assumption that the algebras considered satisfy the Universal Coefficient Theorem (UCT). A technical statement regarding the relation between different K-theoretic invariants (K-theory and KK-theory), the UCT is an assumption (sometimes automatically satisfied) in all the positive classification results for separable, nuclear $\mathrm{C}^{*}$-algebras that have been obtained so far. At the same time, no example of separable, nuclear $\mathrm{C}^{*}$-algebra that does not satisfy the UCT is currently known. This has brought considerable interest to the following UCT problem.

Problem 7.2. Does every separable, nuclear $\mathrm{C}^{*}$-algebra satisfy the UCT?

The UCT has been verified for several important classes of $\mathrm{C}^{*}$-algebras. In fact, it holds for all the separable $\mathrm{C}^{*}$-algebras that can be obtained starting from finite-dimensional and abelian $\mathrm{C}^{*}$-algebras by the standard constructions of $\mathrm{C}^{*}$-algebra theory, such as inductive limits and crossed products by amenable groups. Again, the problem of whether such a class contains in fact all separable, nuclear $\mathrm{C}^{*}$-algebras is currently open. It is clear that the answer to this problem is intimately connected with the problem of finding other methods of constructing nuclear $\mathrm{C}^{*}$-algebras, other than the standard constructions in $\mathrm{C}^{*}$-algebra theory. This provides a connection with model theory, which is a source of a different kind of constructions, generally known as model-theoretic forcing or building models by games.

Another connection of the UCT problem and model theory arises from the following reformulation due to Kirchberg: is the Cuntz algebra $\mathcal{O}_{2}$ uniquely determined by its K-theory among simple, separable, purely infinite $\mathrm{C}^{*}$-algebras? This reformulation can be seen as the problem of whether $\mathrm{O}_{2}$ is the unique model of its theory that omits a certain collection of types. Model theory provides criteria (omitting types theorems) that allow one to decide when a theory admits a model omitting certain types. It is thus clear that these results may be particularly relevant to this problem.

The first natural step towards the possible application of methods from model theory to such problems consists in clarifying the model-theoretic content of notions such as nuclearity, $\mathcal{Z}$-stability, and the Elliott invariant.

7.2. Nuclearity is not elementary. It turns out that the property of being nuclear for $\mathrm{C}^{*}$-algebras is not axiomatizable. Also the more generous property of exactness is not axiomatizable. A $\mathrm{C}^{*}$-algebra is exact if, roughly speaking, it can be approximately represented - as an operator space -into full matrix algebras. A deep result of Kirchberg asserts that a separable $\mathrm{C}^{*}$-algebra is exact if and only if it embeds into a nuclear $\mathrm{C}^{*}$-algebra, which can be chosen to be the Cuntz algebra $\mathcal{O}_{2}$. An arbitrary $\mathrm{C}^{*}$-algebra is exact if and only if all its separable $\mathrm{C}^{*}$-subalgebras are exact. Any nuclear $\mathrm{C}^{*}$-algebra is, in particular, exact. It is also important to notice that exactness (or nuclearity) of a $\mathrm{C}^{*}$-algebra only depends on the underlying operator system structure, and it is inherited by passing to operator subsystems. This has the following implications. For $\mathrm{C}^{*}$-algebras $A, B$, a complete order embedding from $A$ to $B$ is a ucp map $\Phi: A \rightarrow B$ with a ucp inverse $\Psi: \Phi(A) \rightarrow A$. If $A, B$ are $\mathrm{C}^{*}$-algebras, $\Phi: A \rightarrow B$ is a complete order embedding, and $B$ is exact, then $A$ is exact.

Lemma 7.3. Suppose that $A$ is a $\mathrm{C}^{*}$-algebra. If every $\mathrm{C}^{*}$-algebra elementarily equivalent to $A$ is exact, then $A$ is $n$-subhomogeneous for some $n \in \mathbb{N}$.

Proof. Suppose that, for every $n \in \mathbb{N}, A$ is not $n$-subhomogeneous. Thus, for every $n \in \mathbb{N}, A$ has an irreducible representation on a Hilbert space of dimension at least $n$. This allows one to find, for every $n \in \mathbb{N}$, a (not 
necessarily unital) subalgebra $B_{n}$ of $A$ and an ideal $J_{n}$ of $B_{n}$ such that the quotient $B_{n} / J_{n}$ is isomorphic to $M_{n}(\mathbb{C})$. Thus, if $\mathcal{U}$ is a nonprincipal ultrafilter over $\mathbb{N}$, then $A^{\mathcal{U}}$ has $\prod_{\mathcal{U}} B_{n}$ as a $\mathrm{C}^{*}$-subalgebra. Furthermore, $\prod_{\mathcal{U}} J_{n}$ is a closed two-sided ideal of $\prod_{\mathcal{U}} B_{n}$, and the corresponding quotient can be identified with $\prod_{\mathcal{U}}\left(B_{n} / J_{n}\right) \cong$ $\prod_{\mathcal{U}} M_{n}(\mathbb{C})$.

If $H$ is a separable infinite-dimensional Hilbert space, one can consider an increasing sequence of projections $p_{n} \in B(\mathbb{C})$ with $\operatorname{rank}\left(p_{n}\right)=n$ such that $p_{n} \rightarrow 1$ in the strong operator topology. Then, for $n \in \mathbb{N}, p_{n} B(H) p_{n} \cong$ $M_{n}(\mathbb{C})$. Furthermore the map $B(H) \rightarrow \prod_{\mathcal{U}}\left(p_{n} B(H) p_{n}\right) \cong \prod_{\mathcal{U}} M_{n}(\mathbb{C})$ defined by $a \mapsto\left[\left(p_{n} a p_{n}\right)\right]$ is a complete order embedding. Since $B(H)$ is not exact, $\prod_{\mathcal{U}} M_{n}(\mathbb{C})$ is not exact either. Since exactness passes to quotients, this implies that $\prod_{\mathcal{U}} B_{n}$ is not exact, and since exactness passes to subalgebras, $A^{\mathcal{U}}$ is not exact.

The converse of Lemma 7.3 holds as well. Indeed, if $A$ is $n$-subhomogeneous for some $n \in \mathbb{N}$, since the class of $n$-subhomogeneous $\mathrm{C}^{*}$-algebras is axiomatizable, any $\mathrm{C}^{*}$-algebra elementarily equivalent to $A$ is $n$ subhomogeneous, and in particular nuclear, and exact.

It follows from Lemma 7.3 that, if $\mathcal{C}$ is an elementary class of $\mathrm{C}^{*}$-algebras, and $\mathcal{C}$ only consists of exact $\mathrm{C}^{*}$-algebras, then in fact $\mathcal{C}$ only consists of $n$-subhomogeneous $\mathrm{C}^{*}$-algebras for some $n \in \mathbb{N}$. In particular, the class of exact $\mathrm{C}^{*}$-algebras and the class of nuclear $\mathrm{C}^{*}$-algebras are not elementary. The same conclusions apply to several other classes of $\mathrm{C}^{*}$-algebras that are important for the classification programme, such as:

- the class of $\mathrm{C}^{*}$-algebras that can be locally approximated by full matrix algebras, known as uniformly hyperfinite (UHF) algebras;

- the class of $\mathrm{C}^{*}$-algebras that can be locally approximated by finite-dimensional $\mathrm{C}^{*}$-algebras, known as approximately finite-dimensional (AF) algebras.

Similarly, the class of simple $\mathrm{C}^{*}$-algebras is not elementary. Indeed, for every $n \in \mathbb{N}, M_{n}(\mathbb{C})$ is simple. However, if $\mathcal{U}$ is a nonprincipal ultrafilter over $\mathbb{N}$, then the ultraproduct $\prod_{\mathcal{U}} M_{n}(\mathbb{C})$ is not simple: the set of elements $\boldsymbol{a}$ of $\prod_{\mathcal{U}} M_{n}(\mathbb{C})$ with representative sequence satisfying $\lim _{n \rightarrow \mathcal{U}}\left\|x_{n}\right\|_{2}=0$, where $\|\cdot\|_{2}$ denotes the normalized Hilbert-Schmidt norm, is a nontrivial closed two-sided ideal of $\prod_{\mathcal{U}} M_{n}(\mathbb{C})$.

7.3. Infinitary formulas. In order to capture properties such as nuclearity, one needs to consider a more generous notion of "formula". We will therefore introduce infinitary formulas, where one is allowed to take countably infinite "conjunctions and disjunctions", which in this setting are expressions of the form $\sup _{n \in \mathbb{N}} \varphi_{n}$ and $\inf _{n \in \mathbb{N}} \varphi_{n}$ where $\left(\varphi_{n}\right)_{n \in \mathbb{N}}$ is a sequence of formulas subject to certain restrictions. Notice that this construction is not allowed in our previous definition of formulas, in which case one is only allowed to take infima and suprema over a variable. In particular, it is important to keep in mind that infinitary formulas are, in general, not formulas in the strict sense that we have considered so far. To avoid confusions, the formulas as we have previously defined are also called, for completeness, finitary or first-order formulas. The same adjectives should be applied to the notions we have defined in terms of first-order formulas, such as axiomatizable classes, definable predicates, and so on.

Let us now introduce formally infinitary formulas in an arbitrary language $L$ as in the framework considered in Subsection 2.3. In fact, we will only consider a special case of infinitary formulas, which we call sup $\bigvee$ infformulas following [52]. Recall that, if $\varphi(\bar{z})$ is a finitary formula (or, more generally, a definable predicate) and $M$ is an $L$-structure, then the interpretation $\varphi^{M}$ of $\varphi$ in $M$ is a uniformly continuous function. Furthermore, one can find uniform continuity modulus for $\varphi^{M}$ which is independent of $M$, and can be explicitly computed in terms of the uniform continuity of the function and relation symbols in the language and their bounds. We refer to this as the continuity modulus $\varpi^{\varphi}$ of $\varphi$. An (infinitary) sup $\bigvee$ inf-formula is an expression $\varphi(\bar{x})$ of the form

$$
\sup _{\bar{y} \in \bar{D}} \inf _{n \in \mathbb{N}} \psi_{n}(\bar{x}, \bar{y})
$$

where $\bar{x}$ is a tuple of variables with corresponding domains $\bar{D}$, and $\left(\psi_{n}(\bar{x}, \bar{y})\right)_{n \in \mathbb{N}}$ is a sequence of existential $L$-formulas (or existential definable predicates) such that the function $\varpi^{\varphi}(\bar{s}, \bar{r}):=\sup _{n \in \mathbb{N}} \min \left\{\varpi^{\psi_{n}}(\bar{s}, \bar{r}), 1\right\}$ tuples $\bar{s}, \bar{r}$ of positive real numbers satisfies $\varpi^{\varphi}(\bar{s}, \bar{r}) \rightarrow 0$ for $\bar{s} \rightarrow 0$ and $\bar{r} \rightarrow 0$. Given an $L$-structure $M$, one can define the interpretation $\varphi^{M}$ of $\varphi$ in $M$ in the obvious way. The requirement on the continuity moduli guarantees that $\varphi^{M}$ is a uniformly continuous function with modulus $\varpi^{\varphi}$ (independent of $M$ ). It is important to notice that the analog of Los' theorem does not hold in general for sup $\bigvee$ inf-formulas. Observe also that any (finitary) sup inf-formula is, in particular, a sup $\bigvee$ inf-formula. A sup $\bigvee$ inf-sentence will be a sup $\bigvee$ inf-formula with no free variables. Positive sup $\bigvee$ inf-formulas are defined as above, but starting from positive quantifier-free formulas (or definable predicates). 
If now $\mathcal{C}$ is a class of $L$-structure, then we say that $\mathcal{C}$ admits an infinitary sup $\bigvee$ inf-axiomatization if there exists a countable collection of conditions of the form $\varphi \leq r$, where $\varphi$ is a sup $\bigvee$ inf-sentence, such that an $L$-structure $M$ belongs to $\mathcal{C}$ if and only if $\varphi^{M} \leq r$ for every such a condition.

7.4. Infinitary axiomatization of nuclearity. We now remark how important classes of $\mathrm{C}^{*}$-algebras admit an infinitary sup $\bigvee$ inf-axiomatization.

7.4.1. UHF algebras. Recall that a $\mathrm{C}^{*}$-algebra $A$ is $\mathrm{UHF}$ if and only if for every tuple $\bar{a}$ in the unit ball of $A$ and $\varepsilon>0$ there exist $n \in \mathbb{N}$ and a unital copy $M_{n}(\mathbb{C}) \subset A$ such that every element of $\bar{a}$ is at distance at most $\varepsilon$ from the unit ball of $M_{n}(\mathbb{C})$. Recall from Subsection 4.4 that the set of matrix units for a unital copy of $M_{n}(\mathbb{C})$ is definable, being the zeroset of the stable formula $\varphi_{M_{n}(\mathbb{C})}\left(z_{i j}\right)$ given by

$$
\max \left\{\left\|z_{i j} z_{k \ell}-\delta_{j k} z_{i \ell}\right\|,\left\|1-\sum_{j=1}^{n} z_{j j}\right\|,\left|1-\left\|z_{i j}\right\|\right|,\left\|x_{i j}-x_{j i}^{*}\right\|: 1 \leq i, j, k, \ell \leq n\right\} .
$$

Recall also that the set $\mathbb{D}$ of scalar multiples of the identity of norm at most 1 (identified with the set of complex number of absolute value at most 1 ) is also definable. One can thus consider, given $\ell, n \in \mathbb{N}$, the existential definable predicate $\psi_{n}\left(z_{1}, \ldots, z_{\ell}\right)$ given by

$$
\inf _{e_{i j}} \inf _{\lambda_{i j}^{(m)}} \max \left\{\left\|z_{m}-\sum_{i, j=1}^{n} \lambda_{i j}^{(m)} e_{i j}\right\|: m=1,2, \ldots, \ell\right\}
$$

where $e_{i j}$ for $1 \leq i, j \leq n$ range among the matrix units for a unital copy of $M_{n}(\mathbb{C})$ and $\lambda_{i j}^{(m)}$ for $1 \leq i, j \leq n$ and $1 \leq m \leq \ell$ range among the complex numbers of absolute value at most 1 . It is clear that the continuity modulus $\varpi^{\psi_{n}}$ of $\psi_{n}$ satisfies $\varpi^{\psi_{n}}\left(t_{1}, \ldots, t_{n}\right) \leq \max \left\{t_{1}, \ldots, t_{\ell}\right\}$. Therefore we can consider the infinitary $\sup \bigvee$ inf-sentence $\varphi_{\ell}$ given by

$$
\sup _{z_{1}, \ldots, z_{\ell} \in D_{1}} \inf _{n \in \mathbb{N}} \psi_{n}\left(z_{1}, \ldots, z_{\ell}\right) .
$$

It is clear that the sup $\bigvee$ inf-conditions $\varphi_{\ell} \leq 0$ for $\ell \in \mathbb{N}$ indeed provide an infinitary sup $\bigvee$ inf-axiomatization for the class of UHF algebras.

7.4.2. AF algebras. The treatment of $\mathrm{AF}$ algebras is entirely analogous. Indeed, a $\mathrm{C}^{*}$-algebra $A$ is $\mathrm{AF}$ if and only if for every tuple $\bar{a}$ in the unit ball of $A$ and $\varepsilon>0$ there exists a finite-dimensional unital $\mathrm{C}^{*}$-subalgebra $F \subset A$ such that every element of $\bar{a}$ is at distance at most $\varepsilon$ from the unit ball of $F$. One can then consider sup $\bigvee$ inf-conditions defined as above, where one replaced full matrix algebras with arbitrary finite-dimensional $\mathrm{C}^{*}$-algebras.

7.4.3. Nuclear algebras. The proof in the case of nuclearity is similar, but slightly more delicate. Recall that a $\mathrm{C}^{*}$-algebra $A$ is nuclear if and only if for every finite tuple $\bar{a}=\left(a_{1}, \ldots, a_{\ell}\right)$ in the unit ball of $A$ and $\varepsilon>0$ there exist $n \in \mathbb{N}$ and ucp maps $\Phi: A \rightarrow M_{n}(\mathbb{C})$ and $\Psi: M_{n}(\mathbb{C}) \rightarrow A$ such that $\left\|(\Psi \circ \Phi)\left(a_{i}\right)-a_{i}\right\|<\varepsilon$ for $i=1,2, \ldots, \ell$. We have been identifying the field $\mathbb{C}$ of scalars with the set of scalar multiples of the unit of $A$. Similarly, we can canonically identify $M_{n}(\mathbb{C})$ with the subalgebra $M_{n}(\mathbb{C}) \otimes 1 \subset M_{n}(\mathbb{C}) \otimes A \cong M_{n}(A)$.

Consider the relation $R$ on $M_{n}(\mathbb{C}) \times A$ defined, for $\alpha \in M_{n}(\mathbb{C})$ and $a \in A$ of norm at most 1 , by

$$
R(\alpha, a)=\inf _{\Psi}\|\Psi(\alpha)-a\| \text {. }
$$

Here $\Psi$ ranges among all the ucp maps $\Psi: M_{n}(\mathbb{C}) \rightarrow A$. A key step consists in showing that $R$ given by a quantifier-free definable predicate. To see this, one should observe that a linear map $\Psi: M_{n}(\mathbb{C}) \rightarrow A$ is ucp if and only if $\Psi(1)=1$, and the matrix

$$
\sum_{i j} e_{i j} \otimes \Psi\left(e_{i j}\right) \in M_{n}(\mathbb{C}) \otimes A
$$

is positive. As we have shown the set of positive elements of $A$ is quantifier-free definable, and the same holds for the set of positive elements of $M_{n}(\mathbb{C}) \otimes A$. Thus the predicate $R$ is quantifier-free definable as well.

On then needs to show that the relation on $A \times M_{n}(\mathbb{C})$ defined by

$$
S(a, \alpha)=\inf _{\Phi}\|\Phi(a)-\alpha\|
$$


where $\Phi$ range among all the ucp maps $\Phi: A \rightarrow M_{n}(\mathbb{C})$, is given by a quantifier-free definable predicate. This can be shown via the correspondence between completely positive maps $\Phi: A \rightarrow M_{n}(\mathbb{C})$ and positive linear functionals $s_{\Phi}$ on $M_{n}(\mathbb{C}) \otimes A$ such that, for every $a \in A$,

$$
\frac{1}{n} \Phi(a)=\sum_{i j} s_{\Phi}\left(e_{i j} \otimes a\right) e_{i j},
$$

where $\left(e_{i j}\right)$ denote the matrix units of $M_{n}(\mathbb{C})$ [36, Proposition 5.8.5].

Using these facts, one can then consider the existential definable predicate $\psi_{n}\left(z_{1}, \ldots, z_{\ell}\right)$ given by

$$
\inf _{\alpha_{1}, \ldots, \alpha_{\ell}} \max _{m}\left\{S\left(z_{m}, \alpha_{m}\right), R\left(\alpha_{m}, z_{m}\right): m=1,2, \ldots, \ell\right\}
$$

where $\alpha_{1}, \ldots, \alpha_{m}$ range within the unit ball of $M_{n}(\mathbb{C})$. Finally, we have that

$$
\sup _{z_{1}, \ldots, z_{\ell} \in D_{1}} \inf _{n} \psi_{n}\left(z_{1}, \ldots, z_{\ell}\right)
$$

is a $\sup \bigvee$ inf-sentence $\varphi_{\ell}$. The conditions $\varphi_{\ell} \leq 0$ for $\ell \in \mathbb{N}$ witness that the class of nuclear $\mathrm{C}^{*}$-algebras admits an infinitary sup $\bigvee$ inf-axiomatization.

7.4.4. Simple $C^{*}$-algebras. We conclude by showing that the class of simple $\mathrm{C}^{*}$-algebras admits an infinitary sup $\bigvee$ inf-axiomatization. Recall that a $\mathrm{C}^{*}$-algebra is simple if it has no nontrivial closed two-sided ideal. We will use the following characterization of simplicity: a $\mathrm{C}^{*}$-algebra is simple $A$ if and only if for every positive elements $a, d \in A$ such that $\|a\|=1$ and $\|d\| \leq 1 / 2$ there exist $n \in \mathbb{N}$ and $c_{1}, \ldots, c_{n} \in A$ such that $c_{1}^{*} c_{1}+\cdots+c_{n}^{*} c_{n}$ is a contraction, and

see [36, Lemma 5.10.1].

$$
\left\|c_{1}^{*} a c_{1}+\cdots+c_{n}^{*} a c_{n}-d\right\|<\varepsilon
$$

Fix $n \in \mathbb{N}$, and observe that the set of $n$-tuples $\bar{c}=\left(c_{1}, \ldots, c_{n}\right)$ such that $c_{1}^{*} c_{1}+\cdots+c_{n}^{*} c_{n}$ is a contraction, is quantifier-free definable. Indeed, the norm of $c_{1}^{*} c_{1}+\cdots+c_{n}^{*} c_{n}$ is equal to the norm of the matrix column vector

$$
C:=\left[\begin{array}{c}
c_{1} \\
c_{2} \\
\vdots \\
c_{n}
\end{array}\right]
$$

which can be identified with the element

$$
\left[\begin{array}{ccccc}
c_{1} & 0 & 0 & \cdots & 0 \\
c_{2} & 0 & 0 & \cdots & 0 \\
c_{3} & 0 & 0 & \cdots & 0 \\
\vdots & \vdots & \vdots & \ddots & \vdots \\
c_{n} & 0 & 0 & \cdots & 0
\end{array}\right]
$$

of $M_{n}(A)$. Thus we can consider for $n \in \mathbb{N}$ the quantifier-free definable predicate $\psi_{n}(x, y)$ given by

$$
\inf _{z_{1}, \ldots, z_{n}} \max \left\{\left\|z_{1}^{*}\left(x^{*} x\right) z_{1}+\cdots+z_{n}^{*}\left(x^{*} x\right) z_{n}-y^{*} y\right\|,\|y\|-2^{-1 / 2},|1-\|x\||\right\}
$$

where $z_{1}, \ldots, z_{n}$ range among all the $n$-tuples such that $z_{1}^{*} z_{1}+\cdots+z_{n}^{*} z_{n}$ is a contraction. We observe that the continuity modulus $\varpi^{\psi_{n}}$ of $\psi_{n}$ satisfies $\varpi^{\psi_{n}}(t, s) \leq t+s$. Indeed, suppose that $\bar{c}$ is a tuple in a $\mathrm{C}^{*}$-algebra $A$ such that $c_{1}^{*} c_{1}+\cdots+c_{n}^{*} c_{n}$ is a contraction, and $a, b$ are elements of the unit ball of $A$. Denote by $C$ the column vector with entries $c_{1}, \ldots, c_{n}$ defined as above. Let also $a \otimes I_{n}$ be the $n \times n$ matrix in $M_{n}(A)$ with $a$ in the diagonal and zeros elsewhere. Then we have that

$$
\begin{aligned}
& \left|\left\|c_{1}^{*} a^{*} a c_{1}+\cdots+c_{n}^{*} a^{*} a c_{n}\right\|-\left\|c_{1}^{*} b^{*} b c_{1}+\cdots+c_{n}^{*} b^{*} b c_{n}\right\|\right| \\
= & \|\|\left(a \otimes I_{n}\right) C\|-\|\left(b \otimes I_{n}\right) C\|\mid \leq\|\left(a \otimes I_{n}\right) C-\left(b \otimes I_{n}\right) C \| \\
= & \left\|\left((a-b) \otimes I_{n}\right) C\right\| \leq\left\|(a-b) \otimes I_{n}\right\|\|C\| \leq\|a-b\| .
\end{aligned}
$$

This easily gives the above claim on the continuity modulus of $\psi_{n}$.

We can thus consider the sup $\bigvee$ inf-sentence $\varphi$ given by

$$
\sup _{x, y \in D_{1}} \inf _{n \in \mathbb{N}} \psi_{n}(x, y) \text {. }
$$


It is clear from the characterization of simplicity recalled above that the condition $\varphi \leq 0$ witnesses that the class of simple $\mathrm{C}^{*}$-algebras admits an infinitary sup $\bigvee$ inf-axiomatization.

7.5. Model-theoretic forcing. Suppose that $L$ is a language and $\mathcal{C}$ is an elementary class of $L$-structure. We present here the technique of model-theoretic forcing following the presentation from [12]. Alternative approaches can be found in [36, 42, 52].

We suppose that $L$ is separable for $\mathcal{C}$. Recall that this means that the seminorm on $L$-formulas $\varphi\left(x_{1}, \ldots, x_{n}\right)$ defined by

$$
\|\varphi\|=\sup \left\{\varphi^{M}(\bar{a}): M \in \mathcal{C}, \bar{a} \in D_{1}^{M} \times \cdots \times D_{n}^{M}\right\}
$$

is separable. After replacing formulas (or definable predicates) with formulas from a fixed countable dense subset, we can assume that $L$ only contains countably many symbols. For simplicity, we work in the setting of languages containing a single domain of quantification, and where all the bounds for the relation symbols in $L$ are the interval $[0,1]$. The arguments can be adapted to the more general setting a straightforward way.

One can consider a canonical dense set of formulas. This is the collection of restricted $L$-formulas, which are formulas where the only connectives used are among the following ones: $t \mapsto 1-t, t \mapsto t / 2,(t, s) \mapsto s \dot{+} t:=$ $\min \{t+s, 1\}$. Observe that, since we are assuming that the language $L$ only contains countably many function and relation symbols, the set of restricted $L$-formulas is countable. Restricted infinitary sup $\bigvee$ inf-formulas are defined as sup $\bigvee$ inf-formulas, but starting from restricted $L$-formulas.

Let $C$ be a countable set of constant symbols that do not already belong to $L$. Set then $L(C)$ to be the language obtained from $L$ by adding the constant symbols from $C$. We will denote $L(C)$-structures as $M^{+}, N^{+}$, etc. If $M^{+}$is an $L(C)$-structure, the $L$-structure obtained from $M^{+}$by "forgetting" about the interpretation of constants in $C$ (which is called the $L$-reduct of $M^{+}$) is denoted by $M$. In this case, we say that $M^{+}$is an expansion of $M$. An $L(C)$-structure is canonical if the set of interpretations of constants from $C$ is dense.

Let us say that an open condition is an expression of the form $\varphi<r$, where $\varphi$ is a quantifier-free restricted $L(C)$-sentence, and $r \in \mathbb{Q}$. An $L(C)$-structure $M^{+}$in $\mathcal{C}$ satisfies $\varphi<r$ if $\varphi^{M}<r$. A forcing condition $p$ is a finite set of open conditions for which there exists an $L(C)$-structure $M^{+}$whose $L$-reduct $M$ belongs to $\mathcal{C}$, and such that all the open conditions in $p$ are satisfied in $M^{+}$. The set $\mathbb{P}$ of forcing conditions is a countable partially ordered set with respect to reverse inclusion.

We aim at proving the following omitting types theorem, which provides a sufficient (in fact, also necessary) criterion for the existence of structures with certain properties.

Theorem 7.4 (Omitting types). Suppose that $P$ is a property admitting an infinitary sup $\bigvee$ inf-axiomatization given by a countable collection of conditions $\varphi_{n} \leq r_{n}$, where $\varphi_{n}$ is the $\sup \bigvee$ inf-sentence $\sup _{\bar{x}} \psi_{n}(\bar{x})$ and $\psi_{n}(\bar{x})$ $i s$ of the form $\inf _{m \in \mathbb{N}} \inf _{\bar{y}} \sigma_{n, m}(\bar{x}, \bar{y})$ for some quantifier-free definable predicates $\sigma_{n, m}(\bar{x}, \bar{y})$. Suppose that for every forcing condition $q$, every $n \in \mathbb{N}$, every $\varepsilon>0$, and every tuple of constants $\bar{c}$ in $C$, the set of open (infinitary) conditions $q \cup\left\{\psi_{n}(\bar{c})<r_{n}+\varepsilon\right\}$ is satisfied in some $L(C)$-structure whose L-reduct is in $\mathcal{C}$. Then there exists a separable L-structure satisfying $P$.

Let us say that a forcing condition $p$ forces an open condition $\varphi<r$, for some atomic $L(C)$-sentence $\varphi$, in formulas $p \vdash \varphi<r$, if there is $r_{0} \leq r$ such that the open condition $\varphi<r_{0}$ belongs to $p$. We extend the definition of forcing to more general formulas by induction on the complexity [12, Remark 2.3]:

- $p \vdash \frac{1}{2} \varphi<r$ iff $p \vdash \varphi<2 r$;

- $p \vdash(1-\varphi)<r$ iff $\exists s>1-r$ such that for every $q \leq p, q \nvdash \varphi<s$;

- $p \vdash \varphi \dot{+} \psi<r$ iff there exist $s_{0}, s_{1}$ such that $s_{0}+s_{1}<r$ and $p \vdash \varphi<s_{0}$ and $p \vdash \psi<s_{1}$;

- $p \vdash \inf _{n} \varphi_{n}<r$ iff there is $n$ such that $p \vdash \varphi_{n}<r$;

- $p \vdash \inf _{x} \varphi(x)<r$ iff there is $c \in C$ such that $p \vdash \varphi(c)<r$.

This forcing notion satisfy the following properties:

(1) Given $p, q \in \mathbb{P}$ such that $q \leq p$, if $p \vdash \varphi<r$, then $q \vdash \varphi<r$;

(2) Given $p \in \mathbb{P}, \varepsilon>0$, and an $L(C)$-term $\tau$ without variables, there exists $q \leq p$ and $c \in C$ such that $q \vdash d(\tau, c)<\varepsilon$

(3) Given $p \in \mathbb{P}, r>0$, and $L(C)$-terms $\tau$, $\sigma$ without free variables, if $p \vdash d(\sigma, \tau)<r$, then there exists $q \leq p$ such that $q \vdash d(\tau, \sigma)<r$;

(4) Given $p \in \mathbb{P}$, a quantifier-free $L$-formula $\varphi\left(x_{1}, \ldots, x_{\ell}\right)$ with continuity modulus $\varpi^{\varphi}, L(C)$-terms $\tau_{1}, \ldots, \tau_{\ell}, \sigma_{1}, \ldots, \sigma_{\ell}$ without variables, and $\delta_{1}, \ldots, \delta_{\ell}>0$ such that $p \vdash d\left(\tau_{i}, \sigma\right)<\delta$, then there exists $q \leq p$ such that $q \vdash\left|\varphi\left(\sigma_{1}, \ldots, \sigma_{\ell}\right)-\varphi\left(\tau_{1}, \ldots, \tau_{\ell}\right)\right|<\varpi^{\varphi}\left(\delta_{1}, \ldots, \delta_{\ell}\right)$. 
In term of this forcing relation, we define a weak forcing relation as follows. Suppose that $p$ is a forcing condition and $\varphi<r$ is an open condition. Then $p$ weakly forces $\varphi<r$, in formulas $p \vdash^{w} \varphi<r$ if and only if there exists $r^{\prime}<r$ such that for every $q \leq p$ there exists $q^{\prime} \leq q$ such that $q^{\prime} \vdash \varphi<r^{\prime}$.

The following is the main lemma in the proof of Theorem 7.4; see [12, Proposition 4.5]

Lemma 7.5. Suppose that $\varphi$ is the $\sup \bigvee \inf -$ sentence $\inf _{\bar{x}} \psi(\bar{x})$ where $\psi(\bar{x})=\inf _{m} \inf _{\bar{y}} \sigma_{m}(\bar{x}, \bar{y})$. Fix a forcing condition $p \in \mathbb{P}$, and $\varepsilon>0$. Assume that for every forcing condition $q \leq p$ and for every tuple $\bar{c}$, there exists a tuple $\bar{d}$ in $C$ and $m \in \mathbb{N}$ such that $q \cup\left\{\sigma_{m}(\bar{c}, \bar{d})<r\right\}$ is a forcing condition. Then $p \vdash \varphi<r+\varepsilon$.

In order to get an $L(C)$-structure from such a forcing notion, we need to start from a filter on $\mathbb{P}$. As in the order-theoretic terminology, a subset $G$ of $\mathbb{P}$ is a filter if it satisfies the following properties:

(1) if $p, q \in \mathbb{P}$ are such that $q \leq p$ and $q \in G$, then also $p \in G$;

(2) if $n \in \mathbb{N}$ and $p_{1}, \ldots, p_{n} \in G$, then there exists $q \in G$ such that $q \leq p_{i}$ for $i=1,2, \ldots, n$.

Definition 7.6. A filter $G \subset \mathbb{P}$ is generic if for every $L(C)$-sentence $\varphi$ and $\varepsilon>0$ there exist $p \in G$ and $r_{0}, r_{1} \in \mathbb{R}$ such that $p \vdash \varphi<r_{0}$ and $p \vdash 1-\varphi<r_{1}$, where $r_{0}+r_{1}<1+\varepsilon$.

A standard argument in forcing shows that generic filters do exist; see also [12, Proposition 2.12].

Lemma 7.7. Fix $p \in \mathbb{P}$. Then there is a generic filter $G \subset \mathbb{P}$ that contains $p$.

Proof. Fix an enumeration of all the pairs $\left(\varphi_{n}, \varepsilon_{n}\right)$ where $\varphi_{n}$ is an $L(C)$-sentence and $\varepsilon \in \mathbb{Q} \cap(0,+\infty)$. Define a sequence $\left(p_{n}\right)$ in $\mathbb{P}$ as follows. Set $p_{0}:=p$. Assuming that $p_{n}$ has been defined. By definition of the forcing notion, there exist $p_{n+1} \leq p_{n}$ and $r_{0}, r_{1} \in \mathbb{R}$ such that $p_{n+1} \vdash \varphi_{n}<r_{0}, p_{n+1} \vdash\left(1-\varphi_{n}\right)<r_{1}$, and $r_{0}+r_{1}<1+\varepsilon_{n}$. This defines $p_{n+1}$, concluding the recursive construction. Let now $G$ be the filter generated by the sequence $\left(p_{n}\right)$. This is just the set of $q \in \mathbb{P}$ such that $q \geq p_{n}$ for some $n \in \mathbb{N}$. Then $G$ is a generic filter containing $p$.

Suppose now that $G$ is a generic filter for $\mathbb{P}$. If $\varphi<r$ is an open condition, define $G \vdash \varphi<r$ if and only if $p \vdash \varphi<r$ for some $p \in G$. This is equivalent to the assertion that $p \vdash^{w} \varphi<r$ for some $p \in G$ [12, Lemma 2.13]. For an $L(C)$-sentence $\varphi$, set $\varphi^{G}$ to be the infimum of $r$ such that $G \vdash \varphi<r$.

The properties of the forcing notion listed above readily imply the following $[12$, Lemmas 2.14, 2.15]:

(1) If $\varphi$ is an $L(C)$-sentence, then $(1-\varphi)^{G}=1-\varphi^{G}$;

(2) For every $L(C)$-term $\tau$ and $\varepsilon>0$ there exists a term $c \in C$ such that $d(\tau, c)<\varepsilon$;

(3) If $\tau, \sigma$ are $L(C)$-terms without variables, then $d(\sigma, \tau)^{G}=d(\tau, \sigma)^{G}$.

(4) Given an atomic $L(C)$-formula $\varphi\left(x_{1}, \ldots, x_{\ell}\right)$ with continuity modulus $\varpi^{\varphi}, L(C)$-terms $\tau_{1}, \ldots, \tau_{\ell}, \sigma_{1}, \ldots, \sigma_{\ell}$ without variables, and $\delta_{1}, \ldots, \delta_{\ell}>0$, if $d\left(\tau_{i}, \sigma_{i}\right)^{G}<\delta_{i}$ for $i=1,2, \ldots, \ell$, then $\left|\varphi\left(\sigma_{1}, \ldots, \sigma_{\ell}\right)-\varphi\left(\tau_{1}, \ldots, \tau_{\ell}\right)\right|^{G}<$ $\varpi^{\varphi}\left(\delta_{1}, \ldots, \delta_{\ell}\right)$.

Given a generic filter $G$, one can define the corresponding canonical compiled structure $M_{G}^{+}$, whose $L$-reduct is denoted by $M_{G}$, as follows. Consider the set $M_{0}$ of $L(C)$-terms, and define a metric $d_{M_{0}}$ on $M_{0}$ by setting, in the notation above, $d_{M_{0}}\left(t_{0}, t_{1}\right)=\varphi^{G}$, where $\varphi$ is the atomic $L(C)$-sentence $d\left(t_{0}, t_{1}\right)$. Let then $M_{G}$ be the Hausdorff completion of $M_{0}$. By abuse of notation, we identify $M_{0}$ as a subset of $M_{G}$. One can define interpretation of function and relation symbols from $L$ in $M$ as follows. If $c \in C$ then we let $c^{M_{G}^{+}}$be $c$, which belongs to $M_{0}$ as an $L(C)$-term. If $f$ is an $n$-ary function symbol and $t_{1}, \ldots, t_{n} \in M_{0}$ are $L(C)$-terms, then we let $f^{M_{G}^{+}}\left(t_{1}, \ldots, t_{n}\right)$ be the $L(C)$-term $f\left(t_{1}, \ldots, t_{n}\right) \in M_{0}$. Uniform continuity guarantees that $f^{M_{G}^{+}}$extends to a continuous function $f^{M_{G}^{+}}: M_{G} \rightarrow M_{G}$. Similarly, for an $n$-ary relation symbol $R$ and $t_{1}, \ldots, t_{n} \in M_{0}$ are $L(C)$-terms, we let $R^{M_{G}^{+}}\left(t_{1}, \ldots, t_{n}\right)$ be $\varphi^{G}$, where $\varphi$ is the atomic $L(C)$-sentence $R\left(t_{1}, \ldots, t_{n}\right)$. Again, one can then extend $R^{M}$ to the whole of $M_{G}$ by uniform continuity.

The properties of the assignment $\varphi \mapsto \varphi^{G}$ listed above show that $M_{G}^{+}$is indeed a canonical $L(C)$-structure. Furthermore, one can show by induction on the complexity that, if $\varphi$ is an $L(C)$-formula, then $\varphi^{M_{G}^{+}}=\varphi^{G}$.

Proof of Theorem 7.4. Let $G$ be a generic filter for $\mathbb{P}, M_{G}^{+}$be the corresponding compiled structure, and $M_{G}$ is its $L$-reduct. Recall that $P$ is a property admitting an infinitary sup $\bigvee$ inf-axiomatization given by a countable collection of conditions $\varphi_{n} \leq r_{n}$, where $\varphi_{n}$ is the $\sup \bigvee \inf$-sentence $\sup _{\bar{x}} \psi_{n}(\bar{x})$ and $\psi_{n}(\bar{x})$ is of the form $\inf _{m \in \mathbb{N}} \inf _{\bar{y}} \sigma_{n, m}(\bar{x}, \bar{y})$ for some quantifier-free definable predicates $\sigma_{n, m}(\bar{x}, \bar{y})$.

Claim. $M_{G}$ satisfies $P$. 
Observe that, by approximating them, we can assume that the sup $\bigvee$ inf-sentences $\varphi_{n}$ are actually restricted sup $\bigvee$ inf-sentences. Furthermore, after replacing the conditions $\varphi_{n} \leq r_{n}$ for $n \in \mathbb{N}$ with the conditions $\varphi_{n} \leq$ $r_{n}+2^{-m}$ for $n, m \in \mathbb{N}$, it actually suffices to show that the claim holds under the following assumptions: for every forcing condition $p$, every $n \in \mathbb{N}$, and every tuple of constants $\bar{c}$ in $C$, the set of open (infinitary) conditions $p \cup\left\{\psi_{n}(\bar{c})<r_{n}\right\}$ is satisfied in some structure from $\mathcal{C}$. Finally, since we will show that every generic filter works, it suffices to consider the case when $P$ is axiomatized by a single condition $\varphi \leq r$, where $\varphi$ is the infinitary $\sup \bigvee$ inf-formula $\sup _{\bar{x}} \psi(\bar{x})$ and $\psi(\bar{x})$ is the formula $\inf _{m} \inf _{\bar{y}} \sigma_{m}(\bar{x}, \bar{y})$. In this case, we want to prove that the claim holds under the assumption that for every forcing condition $q$, and every tuple of constants $\bar{c}$ from $\mathcal{C}$, there exists an $L$-structure satisfying $\psi(\bar{c})<r$.

Fix $\varepsilon>0$. By the properties of the compiled structure, we have that $\varphi^{M_{G}}=\varphi^{G}$. Furthermore, $\varphi^{G}<r+\varepsilon$ if and only if $p \vdash \varphi<r+\varepsilon$ for some $p \in G$. Fix an arbitrary $p \in G$. Fix a forcing condition $q \leq p$, and a tuple $\bar{c}$. Since $q \cup\{\psi(\bar{c})<r\}$ is satisfiable in an $L(C)$-structure whose $L$-reduct is in $\mathcal{C}$, there exist $k \in \mathbb{N}$ and a tuple $\bar{d}$ in $C$ such that $q^{\prime}:=q \cup\{\sigma(\bar{c}, \bar{d})<r\}$ is a forcing condition. By Lemma 7.5, this shows that $p \vdash \varphi<r+\varepsilon$, concluding the proof.

7.6. Building models by games. One can alternatively present the ideas above using the formalism of "building models by games". This has been developed in classical discrete logic in [57]. A version in the setting of logic for metric structures is considered in [52]. In this setting, one considers game involving two players (Abelard and Eloise). The players alternate turns, and the game runs for infinitely many turns. Abelard starts by playing a forcing condition $p_{0}$, and Eloise has to reply with a forcing condition $p_{1}$ such that $p_{1} \leq p_{0}$, in the sense that every open condition in $p_{0}$ also belongs to $p_{1}$. Abelard then replies with a forcing condition $p_{2}$ containing $p_{1}$, and so on. The game runs for infinitely many turns, producing a chain $p_{0} \geq p_{1} \geq p_{2} \geq \cdots$ of conditions. One then lets $\bar{p}$ be their union.

A play of the game is definitive if, for every atomic $L(C)$-sentence $\varphi$ there exists $r^{\varphi} \in \mathbb{R}$ (only depending on $\varphi)$ such that, for every $L$-structure $M$ in $\mathcal{C}$ satisfying $\bar{p}$ one has that $\varphi^{M}=r^{\varphi}$. In this case, one can define a canonical $L(C)$-structure $M^{+}(\bar{p})$, called the compiled structure, as follows. Consider the set $M_{0}$ of $L(C)$-terms, and define a metric $d_{M_{0}}$ on $M_{0}$ by setting, in the notation above, $d_{M_{0}}\left(t_{0}, t_{1}\right)=r^{\varphi}$, where $\varphi$ is the atomic $L(C)$-sentence $d\left(t_{0}, t_{1}\right)$. Let then $M(\bar{p})$ be the Hausdorff completion of $M_{0}$. By abuse of notation, we identify $M_{0}$ as a subset of $M(\bar{p})$. One can define interpretation of function and relation symbols from $L$ in $M(\bar{p})$ as follows. If $c \in C$ then we let $c^{M}$ be $c$, which belongs to $M_{0}$ as an $L(C)$-term. If $f$ is an $n$-ary function symbol and $t_{1}, \ldots, t_{n} \in M_{0}$ are $L(C)$-terms, then let $f^{M}\left(t_{1}, \ldots, t_{n}\right)$ be the $L(C)$-term $f\left(t_{1}, \ldots, t_{n}\right) \in M_{0}$. Uniform continuity guarantees that $f^{M}$ extends to a continuous function $f^{M}: M \rightarrow M$. Similarly, for an $n$-ary relation symbol $R$ and $t_{1}, \ldots, t_{n} \in M_{0}$ are $L(C)$-terms, one let $R^{M}\left(t_{1}, \ldots, t_{n}\right)$ be, in the notation above, $r^{\varphi}$ where $\varphi$ is the atomic $L(C)$-sentence $R\left(t_{1}, \ldots, t_{n}\right)$. Again, one can then extend $R^{M}$ to the whole of $M$ by uniform continuity.

Then one can reformulate the weak forcing relation as follows [52, Theorem 2.22]. If $p$ is a forcing condition and $\varphi<r$ is an open condition, then $p \vdash^{w} \varphi<r$ if and only if the following holds: suppose that the game has been played up to the $k$-th turn, defining forcing conditions $p_{0} \geq p_{1} \geq \cdots \geq p_{k}$ such that $p_{k} \leq p$. Then, regardless of Abelard's moves, Eloise can keep playing the game in such a way that the game is definitive and the compiled structure $M(\bar{p})$ satisfies the open condition $\varphi<r$. In other words, the position $\left(p_{0}, \ldots, p_{k}\right)$ is a winning position for Eloise in the game $G(\bar{p})$ whose winning conditions for Eloise are that the game is definitive and the compiled structure satisfies the open condition $\varphi<r$.

7.7. Forcing and the UCT problem. The omitting types theorem (Theorem 7.4) provides a method of constructing $\mathrm{C}^{*}$-algebras, which is different from any other the standard constructions in $\mathrm{C}^{*}$-algebra theory. It is therefore reasonable to expect that it might at least have some bearing on the UCT question; see Subsection 7.1. Indeed, one can use Theorem 7.4 to provide a "concrete" reformulation of the UCT question, which in some sense can be seen as the combinatorial core - or a combinatorial core - of such a problem.

We will consider a reformulation of the UCT problem due to Kirchberg [70, Corollary 8.4.6]. This reformulation asserts that every separable nuclear $\mathrm{C}^{*}$-algebra satisfies the UCT if and only if $\mathcal{O}_{2}$ is uniquely characterized among separable, nuclear, simple, and purely infinite $\mathrm{C}^{*}$-algebras by its Elliott invariant. Since for purely infinite $\mathrm{C}^{*}$-algebras the trace simplex is empty, this is equivalent to the assertion that $\mathcal{O}_{2}$ is the unique separable, nuclear, simple, and purely infinite $\mathrm{C}^{*}$-algebra with trivial $K_{0}$ and $K_{1}$ groups.

We claim that the class of simple, purely infinite $\mathrm{C}^{*}$-algebra with trivial $K_{0}$-group is elementary, and in fact sup inf-axiomatizable. We have seen in Subsection 3.5 that the class of simple, purely infinite $\mathrm{C}^{*}$-algebras is elementary, and the proof there shows that it is in fact sup inf-axiomatizable. For a simple, purely infinite 
$\mathrm{C}^{*}$-algebra $A$, the $K_{0}$-group of $A$ is trivial if and only if any two nonzero projections of $A$ are Murray-von Neumann equivalent [70, Proposition 4.1.4]. We have shown in Subsection 3.4 that the relation of Murray-von Neumann equivalent is definable, as witnessed by a existential definable predicate. This easily shows that the class of simple, purely infinite $\mathrm{C}^{*}$-algebras with trivial $K_{0}$-group is sup inf-definable.

We now claim that there is a sup inf-axiomatizable class $\mathcal{C}$ such that the set of separable nuclear $\mathrm{C}^{*}$-algebras that belong to $\mathcal{C}$ are precisely the separable, nuclear, simple, and purely infinite $\mathrm{C}^{*}$-algebras with trivial $K_{0}$ and $K_{1}$ groups. Let $A$ be a separable, nuclear, simple, and purely infinite $\mathrm{C}^{*}$-algebra $A$. Then, the $K_{1}$-group of $A$ is trivial if and only if the unitary group $U(A)$ is connected [70, Proposition 4.1.15]. Furthermore, $A$ absorbs tensorially the Cuntz algebra $\mathcal{O}_{\infty}$ by Kirchberg's $\mathcal{O}_{\infty}$-absorption theorem [59]. Thus by [66, Theorem 3.1], any element in the connected component $U_{0}(A)$ of the identity in $U(A)$ is connected to the identity by a path of length at most $2 \pi$. Since any unitary at distance less than 2 from the identity is in $U_{0}(A)$, this allows one to conclude that $A$ has connected unitary group if and only if it satisfies the sup inf-condition

$$
\sup _{u \text { unitary }} \inf _{1}, v_{2}, v_{3} \text { unitaries } \max \left\{\left\|u-v_{1}\right\|,\left\|v_{1}-v_{2}\right\|,\left\|v_{2}-v_{3}\right\|,\left\|v_{3}-1\right\|\right\} \leq 7 / 4
$$

Thus adding such a condition to the set of axioms for simple, purely infinite $\mathrm{C}^{*}$-algebras with trivial $K_{0}$-group gives an axiomatization for an elementary class $\mathcal{C}$ as desired.

Let $\mathcal{C}_{\text {nuc }}$ be the class of nuclear $\mathrm{C}^{*}$-algebras in $\mathcal{C}$. Since $\mathcal{C}$ is sup inf-axiomatizable, and nuclearity admits an infinitary sup $\bigvee$ inf-axiomatization, the class $\mathcal{C}_{\text {nuc }}$ admits an infinitary sup $\bigvee$ inf-axiomatization. This is just obtained by adding to the axioms of $\mathcal{C}$ the $\sup \bigvee$ inf-condition defining nuclearity. Let $\varphi_{n}^{\mathcal{C}} \leq 0$ for $n \in \mathbb{N}$ be such conditions, which can be explicitly extracted from the discussions above. For each $\bar{n} \in \mathbb{N}, \varphi_{n}^{\mathcal{C}}$ is a

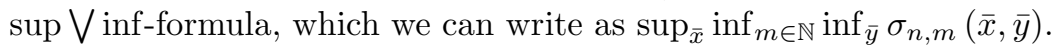

Suppose now that $A$ is a separable $\mathrm{C}^{*}$-algebra from $\mathcal{C}_{\text {nuc }}$. Then by Kirchberg's $\mathcal{O}_{2}$-absorption theorem, $A$ is isomorphic to $\mathcal{O}_{2}$ if and only if $A$ absorbs $\mathcal{O}_{2}$ tensorially. By Theorem 6.5 , this is equivalent to the assertion that, for every positive quantifier-free formula $\theta(\bar{y})$, if $\psi_{\theta}$ is the positive sup inf-sentence

$$
\sup _{\bar{x}} \inf _{\bar{y}} \max \left\{\theta(\bar{y}),\left\|x_{i} y_{j}-y_{j} x_{i}\right\|: i, j\right\}
$$

then $\psi_{\theta}^{\mathcal{O}_{2}} \leq \psi_{\theta}^{A}$. Thus $A$ is not isomorphic to $\mathcal{O}_{2}$ if and only if there exists a sup inf-sentence $\psi_{\theta}$ such that $\psi_{\theta}^{\mathcal{O}_{2}}>\psi_{\theta}^{A}$. In view of the above discussion and the omitting types theorem (Theorem 7.4) applied to the class of $\mathrm{C}^{*}$-algebras regarded as $L^{\mathrm{C}^{*}}$-structures, one can provide the following sufficient criterion to establish that the UCT fails.

Theorem 7.8. Assume that there exists a quantifier-free formula $\theta$ such that the following holds. For every finite set of open quantifier-free conditions $\psi_{i}(\bar{x}, \bar{y}, \bar{z})<r_{i}$ for $i=1,2, \ldots, \ell$ which are realized in some $C^{*}$-algebra, there exist $m \in \mathbb{N}$, a $C^{*}$-algebra $A$ satisfying $\psi_{\theta}^{A}<\psi_{\theta}^{\mathcal{O}_{2}}$, and tuples $\bar{a}, \bar{b}, \bar{c}$ in $A$, satisfying $\psi_{i}(\bar{a}, \bar{b}, \bar{c})<r_{i}$, and $\sigma_{n, m}(\bar{a}, \bar{b})<\varepsilon$. Then the UCT fails.

\section{FURTHER RESULTS AND OUTLOOK}

For reasons of space, we have omitted in the above discussion many important directions of applications of model theory to operator algebras. For the sake of completeness, we mention here some of such directions.

Von Neumann factors. Finite Von Neumann factors and, more generally, tracial von Neumann algebras also fit in the framework of first order logic for metric structures. Recall that a tracial von Neumann algebra is a von Neumann algebra $M$ endowed with a distinguished faithful normal tracial state $\tau$. A tracial von Neumann algebra is a finite factor if it has trivial center. In this case, the faithful normal tracial state $\tau$ is uniquely determined by $M$. A $\mathrm{II}_{1}$ factor is an infinite-dimensional finite factor.

In order to regard tracial von Neumann algebras as structures, one can consider the language of $\mathrm{C}^{*}$-algebras with an additional relation symbol $\tau$ to be interpreted as the given trace. In this case, the relation symbol for the norm should be interpreted as the 2-norm $\|x\|_{\tau}=\tau\left(x^{*} x\right)^{1 / 2}$ associated with the trace $\tau$. Consistently, the canonical binary relation symbol for the metric should be interpreted as the metric associated with such a norm. The domains $D_{n}$ for $n \in \mathbb{N}$ should still be interpreted as the closed balls with respect to the operator norm. This perspective has been used in [38, 39] to answer to a question of McDuff from [62] on the number of isomorphism classes of relative commutants $M^{\prime} \cap M^{\mathcal{U}}$ associated with nonprincipal ultrafilters $\mathcal{U}$ over $\mathbb{N}$ for a given separable $\mathrm{II}_{1}$ factor $M$. As in the case of $\mathrm{C}^{*}$-algebras, the Continuum Hypothesis $(\mathrm{CH})$ implies that all such relative commutants are isomorphic, while the negation of $\mathrm{CH}$ implies that there exist at least two nonisomorphic such relative commutants. The model-theoretic study of factors has been further pursued in $[15,34,40,53]$, where it 
is shown that the set of separable models of a consistent theory of $\mathrm{II}_{1}$ factors has size continuum, the theory of tracial von Neumann algebras does not have a model companion, the Connes Embedding Problem is equivalent to the assertion that the hyperfinite $\mathrm{II}_{1}$ factor $\mathcal{R}$ is existentially closed, and that there exists a continuum of distinct theories of $\mathrm{II}_{1}$ factors.

Compact Hausdorff spaces. In a series of papers going back to the 1980s [5-8], Bankston introduced dual notions to fundamental notions in model theory (elementary equivalence, elementary embedding, ultraproduct), and applied such notions to the study of compact Hausdorff spaces. It has been observed in [25] that, if one replaces a compact Hausdorff space $X$ with the abelian $\mathrm{C}^{*}$-algebra $C(X)$ of continuous functions over $X$, then the notions introduced by Bankston coincide with the usual notions from model theory for metric structures where $C(X)$ is regarded as a structure in the language of $\mathrm{C}^{*}$-algebras. This perspective has been used in [25, 52] to show that, if $\mathbb{P}$ is the pseudoarc (the unique hereditarily indecomposable, chainable, metrizable continuum), then $C(\mathbb{P})$ is existentially closed - in Bankston's terminology, $\mathbb{P}$ is co-existentially closed - and it is the prime model of its theory. Furthermore, for zero-dimensional compact Hausdorff spaces, elementary equivalence is equivalent to elementary equivalence of the associated Boolean algebras of clopen sets [26]. If the spaces have no isolated points, similar conclusions apply to countable saturation [26].

Enforceable operator algebras. The framework of model-theoretic forcing can be used to define the notion of enforceable structure. Let $\mathcal{C}$ be an elementary class of $L$-structure, and consider the game between Abelard and Eloise defined in Subsection 7.6. One then says that a property $P$ of $L$-structures is enforceable if Eloise has a winning strategy when her winning conditions require the compiled structure to satisfy $P$. This can be seen as a model-theoretic notion of genericity for $L$-structures satisfying $P$ within the class $\mathcal{C}$. An $L$-structure $M$ is enforceable if the property of being isomorphic to $M$ is enforceable.

Many outstanding open problems in operator algebra theory can be reformulated as the assertion that the known examples of strongly self-absorbing $\mathrm{C}^{*}$-algebras are enforceable within a suitable class of $\mathrm{C}^{*}$-algebras [52]. For instance, the Kirchberg embedding problem, asking whether every $\mathrm{C}^{*}$-algebra embeds into an ultrapower of the Cuntz algebra $\mathcal{O}_{2}$, is equivalent to the assertion that $\mathcal{O}_{2}$ is enforceable in the class of all $\mathrm{C}^{*}$-algebras. Similarly, the MF problem of Blackadar and Kirchberg, asking whether every stably finite $\mathrm{C}^{*}$-algebra embeds into an ultrapower of the rational UHF algebra $\mathcal{Q}=\bigotimes_{n \in \mathbb{N}} M_{n}(\mathbb{C})$, is equivalent to the assertion that $Q$ is enforceable within the class of stably finite $\mathrm{C}^{*}$-algebras. Finally, the assertion that every stably finite projectionless $\mathrm{C}^{*}$-algebra embeds into an ultrapower of the Jiang-Su algebra $\mathcal{Z}$ is equivalent to the assertion that $\mathcal{Z}$ is enforceable within the class of stably finite projectionless $\mathrm{C}^{*}$-algebras.

This framework can also be applied in the context of $\mathrm{II}_{1}$ factors. In this case, the Connes Embedding Problem, asking whether every $\mathrm{II}_{1}$ factor embeds into an ultrapower of the hyperfinite $\mathrm{II}_{1}$ factor $\mathcal{R}$, turns out to be equivalent to the assertion that $\mathcal{R}$ is enforceable within the class of $\mathrm{II}_{1}$ factors.

Actions of compact (quantum) groups on $\mathbf{C}^{*}$-algebras. The equivariant theory of $\mathrm{C}^{*}$-algebras studies $\mathrm{C}^{*}$-algebras endowed with a distinguished continuous action of a locally compact group $G(G$-C*algebras). The case which is best understood is when the acting group $G$ is finite or, more generally, compact. It is clear that, when $G$ is finite, one can regard $G$-C $C^{*}$-algebras as structures in the language $L_{G}^{\mathrm{C}^{*}}$ obtained from the language of $\mathrm{C}^{*}$-algebras by adding unary functions symbols $\alpha_{g}$ for $g \in G$, to be interpreted as the automorphism of the given $C^{*}$-algebras that define the $G$-action. More generally, as shown in [47], for an arbitrary compact groups $G$, $G$-C*-algebras fit into the framework of first order logic for metric structures described above. To see this, one should notice the following. Suppose that $A$ is a $\mathrm{C}^{*}$-algebra, and $\alpha$ is a continuous action of $G$ on $A$. One can regard $\alpha$ as a ${ }^{*}$-homomorphism $\alpha: A \rightarrow C(G, A) \cong C(G) \otimes A, a \mapsto\left(g \mapsto \alpha_{g}(a)\right)$. For every finite-dimensional irreducible representation $\pi \in \operatorname{Rep}(\pi)$ of $G$ one can consider the span $C(G)_{\pi} \subset C(G)$ of the matrix units of $\pi$. The subspace $A_{\pi}=\left\{a \in A: \alpha(a) \in C(G)_{\pi} \otimes A\right\}$ is called spectral subspace of $\alpha$ associated with $\pi$. The union of $A_{\pi}$ when $\pi$ varies among all the finite-dimensional representations of $G$ is a dense ${ }^{*}$-subalgebra of $A$ (Podleś algebra) [68]. One can regard the $G$-C $C^{*}$-algebra $(A, \alpha)$ as a two-sorted structure, with a sort for $A$ and a sort for $C(G) \otimes A$. The language of $G$-C $C^{*}$-algebras is endowed with domains $D_{\pi}$ for $\pi \in \operatorname{Rep}(\pi)$, to be interpreted in $A$ as $A_{\pi}$ and in $C(G) \otimes A$ as $C(G)_{\pi} \otimes A_{\pi}$. It is shown in [47] that $G$-C $\mathrm{C}^{*}$-algebras form an axiomatizable class in such a language. This perspective, and the corresponding notion of positive existential embedding, has been used implicitly in [9] and explicitly in [48] to give a model-theoretic characterization of the Rokhlin property for $G$-C*-algebras. In turn, this characterization has been used to provide a unified approach to several preservation results for fixed point algebras and crossed products with respect to Rokhlin actions. More generally, a model-theoretic characterization of Rokhlin dimension is considered in [48]. This is applied 
to obtain preservation results of finite nuclear dimension and finite composition rank for fixed point algebras and crossed products with respect to actions with finite Rokhlin dimension.

More generally, the theory can be developed in the context of actions of compact quantum groups on $\mathrm{C}^{*}$ algebras. A quantum group $\mathbb{G}$ is a $\mathrm{C}^{*}$-algebraic object which formally satisfies the same properties (save from being abelian) as the $\mathrm{C}^{*}$-algebra $C(G)$ associated with a classical compact group $G$ endowed with the comultiplication operation $\Delta: C(G) \rightarrow C(G \times G) \cong C(G) \otimes C(G), f \mapsto((s, t) \mapsto f(s t))$. Continuous actions of a compact quantum group $\mathbb{G}$ on $\mathrm{C}^{*}$-algebras ( $\mathbb{G}-\mathrm{C}^{*}$-algebras) can be defined in closed parallel with the classical case. It is also shown in [47] that $\mathbb{G}-\mathrm{C}^{*}$-algebras form an axiomatizable class in a suitable language, very similar to the one described above for classical compact groups. This point of view has been used, implicitly in [10] and explicitly in [47], to generalize the notions of Rokhlin property and Rokhlin dimension to the quantum setting, as well as virtually all known preservation results of regularity properties under fixed point algebras and crossed products by actions with finite Rokhlin dimension.

\section{REFERENCES}

1. Charles A. Akemann and Gert K. Pedersen, Central sequences and inner derivations of separable $C^{*}$ algebras, American Journal of Mathematics 101 (1979), no. 5, 1047-1061.

2. Goulnara Arzhantseva and Liviu Paunescu, Almost commuting permutations are near commuting permutations, Journal of Functional Analysis 269 (2015), no. 3, 745-757.

3. Linear sofic groups and algebras, Transactions of the American Mathematical Society 369 (2017), no. 4, 2285-2310.

4. Micheal F. Atiyah and Hirzebruch Hirzebruch, Riemann-Roch theorems for differentiable manifolds, Bulletin of the American Mathematical Society 65 (1959), no. 4, 276-281.

5. Paul Bankston, Co-elementary equivalence, co-elementary maps, and generalized arcs, Proceedings of the American Mathematical Society 125, no. 12, 3715-3720.

6. __ A hierarchy of maps between compacta, The Journal of Symbolic Logic 64, no. 4, 1628-1644.

7. Some applications of the ultrapower theorem to the theory of compacta, Applied Categorical Structures. 8, no. 1, 45-66.

8. __ Reduced Coproducts of Compact Hausdorff Spaces, The Journal of Symbolic Logic 52 (1987), no. 2, 404-424.

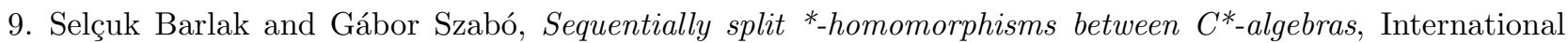
Journal of Mathematics 27 (2016), no. 13.

10. Selçuk Barlak, Gábor Szabó, and Christian Voigt, The spatial Rokhlin property for actions of compact quantum groups, Journal of Functional Analysis 272 (2017), no. 6, 2308-2360.

11. Itaï Ben Yaacov, Alexander Berenstein, C. Ward Henson, and Alexander Usvyatsov, Model theory for metric structures, Model theory with applications to algebra and analysis. Vol. 2, London Mathematical Society Lecture Note Series, vol. 350, Cambridge University Press, 2008, pp. 315-427.

12. Itaï Ben Yaacov and José Iovino, Model theoretic forcing in analysis, Annals of Pure and Applied Logic 158 (2009), no. 3, 163-174.

13. Bruce Blackadar, Operator algebras, Encyclopaedia of Mathematical Sciences, vol. 122, Springer-Verlag, Berlin, 2006.

14. Bruce Blackadar and Eberhard Kirchberg, Generalized inductive limits of finite-dimensional $C^{*}$-algebras, Mathematische Annalen 307 (1997), no. 3, 343-380.

15. Rémi Boutonnet, Ionut Chifan, and Adrian Ioana, II factors with non-isomorphic ultrapowers, Duke Mathematical Journal, in press.

16. Lawrence G. Brown, Ronald G. Douglas, and Peter A. Fillmore, Extensions of C*-algebras and K-homology, Annals of Mathematics. Second Series 105 (1977), no. 2, 265-324.

17. Lawrence $\mathrm{G}$ Brown and Gert Pedersen, $C^{*}$-algebras of real rank zero, Journal of Functional Analysis 99 , no. 1, 131-149.

18. Valerio Capraro and Martino Lupini, Introduction to Sofic and hyperlinear groups and Connes' embedding conjecture, Lecture Notes in Mathematics, vol. 2136, Springer, 2015, With an appendix by Vladimir Pestov.

19. Kevin Carlson, Enoch Cheung, Ilijas Farah, Alexander Gerhardt-Bourke, Bradd Hart, Leanne Mezuman, Nigel Sequeira, and Alexander Sherman, Omitting types and AF algebras, Archive for Mathematical Logic 53 (2014), no. 1-2, 157-169.

20. José R. Carrión, Marius Dadarlat, and Caleb Eckhardt, On groups with quasidiagonal $C^{*}$-algebras, Journal of Functional Analysis 265 (2013), no. 1, 135-152. 
21. Paul J. Cohen, The independence of the continuum hypothesis, Proceedings of the National Academy of Sciences of the United States of America 50 (1963), 1143-1148.

22. _ The independence of the continuum hypothesis. II, Proceedings of the National Academy of Sciences of the United States of America 51 (1964), 105-110.

23. Samuel Coskey and Ilijas Farah, Automorphisms of corona algebras, and group cohomology, Transactions of the American Mathematical Society 366 (2014), no. 7, 3611-3630.

24. Joachim Cuntz, Simple $C^{*}$-algebras generated by isometries, Communications in Mathematical Physics $\mathbf{5 7}$ (1977), no. 2, 173-185.

25. Christopher J. Eagle, Isaac Goldbring, and Alessandro Vignati, The pseudoarc is a co-existentially closed continuum, Topology and its Applications 207 (2016), 1-9.

26. Christopher J. Eagle and Alessandro Vignati, Saturation and elementary equivalence of $C^{*}$-algebras, Journal of Functional Analysis 269 (2015), no. 8, 2631-2664.

27. Gábor Elek and Endre Szabó, Sofic groups and direct finiteness, Journal of Algebra 280 (2004), no. 2, 426-434.

28. Sofic representations of amenable groups, Proceedings of the American Mathematical Society 139 (2011), no. 12, 4285-4291.

29. George Elliott and Andrew Toms, Regularity properties in the classification program for separable amenable $C^{*}$-algebras, Bulletin of the American Mathematical Society 45 (2008), no. 2, 229-245.

30. George A Elliott, On the classification of inductive limits of sequences of semisimple finite-dimensional algebras, Journal of Algebra 38 (1976), no. 1, 29-44.

31. George A. Elliott, The classification problem for amenable $C^{*}$-algebras, Proceedings of the International Congress of Mathematicians, Vol. 1, 2 (Zürich, 1994), Birkhäuser, Basel, 1995, pp. 922-932.

32. Ilijas Farah, All automorphisms of all Calkin algebras, Mathematical Research Letters 18 (2011), no. 3, 489-503.

33. _ All automorphisms of the Calkin algebra are inner, Annals of Mathematics 173 (2011), no. 2, 619-661.

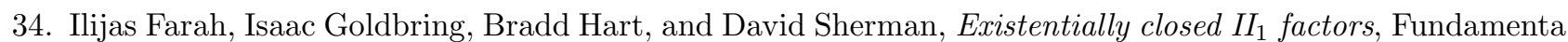
Mathematicae 233 (2016), no. 2, 173-196.

35. Ilijas Farah and Bradd Hart, Countable saturation of corona algebras, Comptes Rendus Mathématiques de l'Académie des Sciences 35 (2013), no. 2, 35-56.

36. Ilijas Farah, Bradd Hart, Martino Lupini, Leonel Robert, Aaron Tikuisis, Alessandro Vignati, and Wilhelm Winter, Model theory of $C^{*}$-algebras, arXiv:1602.08072 (2016).

37. Ilijas Farah, Bradd Hart, Mikael Rørdam, and Aaron Tikuisis, Relative commutants of strongly self-absorbing $C^{*}$-algebras, Selecta Mathematica 23 (2017), no. 1, 363-387.

38. Ilijas Farah, Bradd Hart, and David Sherman, Model theory of operator algebras I: stability, Bulletin of the London Mathematical Society 45 (2013), no. 4, 825-838.

39. Model theory of operator algebras II: model theory, Israel Journal of Mathematics 201 (2014), no. 1, $477-505$.

40. _ Model theory of operator algebras III: elementary equivalence and $I_{1}$ factors, Bulletin of the London Mathematical Society 46 (2014), no. 3, 609-628.

41. Ilijas Farah and Ilan Hirshberg, The Calkin algebra is not countably homogeneous, Proceedings of the American Mathematical Society 144, no. 12, 5351-5357.

42. Ilijas Farah and Menachem Magidor, Omitting types in logic of metric structures, Journal of Mathematical Logic, to appear.

43. Ilijas Farah and Paul McKenney, Homeomorphisms of Cech-Stone remainders: the zero-dimensional case, Proccedings of the American Mathematical Society, to appear.

44. Ilijas Farah, Paul McKenney, and Ernest Schimmerling, Some calkin algebras have outer automorphisms, Archive for Mathematical Logic 52, no. 5, 517-524.

45. Ilijas Farah and Saharon Shelah, A dichotomy for the number of ultrapowers, Journal of Mathematical Logic 10 (2010), no. 1-2, 45-81.

46. _ Rigidity of continuous quotients, Journal of the Institute of Mathematics of Jussieu 15 (2016), no. 1, 1-28.

47. Eusebio Gardella, Mehrdad Kalantar, and Martino Lupini, Rokhlin dimension for compact quantum group actions, arXiv:1703.10999.

48. Eusebio Gardella and Martino Lupini, Equivariant logic and applications to $C^{*}$-dynamics, arXiv:1608.05532 (2016). 
49. Lev Glebsky, Almost commuting matrices with respect to normalized Hilbert-Schmidt norm, arXiv:1002.3082 (2010).

50. James G. Glimm, On a certain class of operator algebras, Transactions of the American Mathematical Society 95 (1960), no. 2, 318-340.

51. Kurt Gödel, The Consistency of the Continuum Hypothesis, Annals of Mathematics Studies, no. 3, Princeton University Press, Princeton, N. J., 1940.

52. Isaac Goldbring, Enforceable operator algebras, arXiv:1706.09048 (2017).

53. Isaac Goldbring, Bradd Hart, and Thomas Sinclair, The theory of tracial von Neumann algebras does not have a model companion, Journal of Symbolic Logic 78 (2013), no. 3, 1000-1004.

54. Isaac Goldbring and Thomas Sinclair, Robinson forcing and the quasidiagonality problem, International Journal of Mathematics 28, no. 2, 1750008, 15.

55. _ On Kirchberg's embedding problem, Journal of Functional Analysis 269 (2015), no. 1, $155-198$.

56. Mikhael Gromov, Endomorphisms of symbolic algebraic varieties, Journal of the European Mathematical Society 1 (1999), no. 2, 109-197.

57. Wilfrid Hodges, Building models by games, London Mathematical Society Student Texts, vol. 2, Cambridge University Press, Cambridge, 1985.

58. Xinhui Jiang and Hongbing $\mathrm{Su}$, On a simple unital projectionless $C^{*}$-algebra, American Journal of Mathematics 121 (1999), no. 2, 359-413.

59. Eberhard Kirchberg and N. Christopher Phillips, Embedding of exact $C^{*}$-algebras in the Cuntz algebra $\mathcal{O}_{2}$, Journal für die reine und angewandte Mathematik 525 (2000), 17-53.

60. Eberhard Kirchberg and Mikael Rørdam, Infinite non-simple $C^{*}$-algebras: absorbing the Cuntz algebra $\mathcal{O}_{\infty}$, Advances in Mathematics 167 (2002), no. 2, 195-264.

61. Terry A. Loring, Lifting solutions to perturbing problems in $C^{*}$-algebras, Fields Institute Monographs, vol. 8, American Mathematical Society, Providence, RI, 1997.

62. Dusa McDuff, Central sequences and the hyperfinite factor, Proceedings of the London Mathematical Society. Third Series 21 (1970), 443-461.

63. Narutaka Ozawa, About the QWEP conjecture, International Journal of Mathematics 15 (2004), no. 5, 501-530.

64. Gert K. Pedersen, Analysis now, Graduate Texts in Mathematics, vol. 118, Springer-Verlag, New York, 1989.

65. Vladimir G. Pestov, Hyperlinear and sofic groups: A brief guide, Bulletin of Symbolic Logic 14 (2008), no. 04, 449-480.

66. N. Christopher Phillips, Real rank and exponential length of tensor products with $\mathcal{O}_{\infty}$, Journal of Operator Theory 47 (2002), no. 1, 117-130.

67. N. Christopher Phillips and Nik Weaver, The Calkin algebra has outer automorphisms, Duke Mathematical Journal 139 (2007), no. 1, 185-202.

68. Piotr Podleś, Symmetries of quantum spaces. Subgroups and quotient spaces of quantum $\mathrm{SU}_{2}$ and $\mathrm{SO}_{3}$ groups, Communications in Mathematical Physics 170 (1995), no. 1, 1-20.

69. Iain Raeburn and Dana P. Williams, Morita equivalence and continuous-trace C*-algebras, Mathematical Surveys and Monographs, vol. 60, American Mathematical Society, Providence, RI, 1998.

70. Mikael Rørdam, Classification of nuclear $C^{*}$-algebras, Encyclopaedia of Mathematical Sciences, vol. 126, Springer-Verlag, Berlin, 2002.

71. Jonathan Rosenberg, Algebraic K-theory and its applications, Graduate Texts in Mathematics, vol. 147, Springer-Verlag, New York, 1994.

72. Claude Schochet, Algebraic topology and $C^{*}$-algebras, C*-algebras: 1943-1993, Contemp. Math., vol. 167, Amer. Math. Soc., Providence, RI, 1994, pp. 218-231.

73. Joseph R. Shoenfield, The problem of predicativity, Essays on the foundations of mathematics, Magnes Press, Hebrew Univ., Jerusalem, 1961, pp. 132-139.

74. Gábor Szabó, Strongly self-absorbing $C^{*}$-dynamical systems, Transactions of the American Mathematical Society, to appear.

75. _ Strongly self-absorbing $C^{*}$-dynamical systems, II, Journal of Noncommutative Geometry, to appear.

76. Strongly self-absorbing $C^{*}$-dynamical systems, III, Advances in Mathematics 316, 356-380.

77. Andreas Thom, Sofic groups and diophantine approximation, Communications on Pure and Applied Mathematics 61 (2008), no. 8, 1155-1171.

78. About the metric approximation of Higman's group, Journal of Group Theory 15 (2012), no. 2, 301-310. 
79. Stevo Todorcevic, Introduction to Ramsey spaces, Annals of Mathematics Studies, vol. 174, Princeton University Press, Princeton, NJ, 2010.

80. Andrew Toms, An infinite family of non-isomorphic $C^{*}$-algebras with identical K-theory, Transactions of the American Mathematical Society 360 (2008), no. 10, 5343-5354.

81. Andrew S. Toms, Comparison theory and smooth minimal $C^{*}$-dynamics, Communications in Mathematical Physics 289 (2009), no. 2, 401-433.

82. Andrew S. Toms and Wilhelm Winter, Strongly self-absorbing $C^{*}$-algebras, Transactions of the American Mathematical Society 359 (2007), no. 8, 3999-4029.

83. Alessandro Vignati, Nontrivial homeomorphisms of Cech-Stone remainders, Münster Journal of Mathematics 10 (2017), no. 1, 189-200.

84. Dan Voiculescu, Asymptotically commuting finite rank unitary operators without commuting approximants, Acta Universitatis Szegediensis 45 (1983), no. 1-4, 429-431.

Martino Lupini, Mathematics Department, California Institute of Technology, 1200 East California Boulevard, Mail Code 253-37, Pasadena, CA 91125

E-mail address: lupini@caltech.edu

URL: http:n//www.lupini.org/ 